\title{
BATF3-dependent induction of IL-27 in B cells bridges the innate and adaptive
}

\section{stages of the antibody response}

Short title: B cells produce IL-27 to mediate the antibody response

One-sentence summary: B cells integrate innate TLR and adaptive CD40 signals to induce BATF3 transcription factor for production of IL-27, which together with INFy optimizes antibody responses.

Authors: Hui $\operatorname{Yan}^{1}$, Rui Wang ${ }^{1,2,3}$, Jingwei Wang ${ }^{1,2,4}$, Shuai Wu ${ }^{1,3}$, Maria Fernandez ${ }^{1}$, Carlos E. Rivera ${ }^{1}$, Christian Cervantes ${ }^{1}$, Justin B. Moroney ${ }^{1}$, Xiao-Dong Li ${ }^{1}$, Nu Zhang ${ }^{1}$, Hong Zan ${ }^{1}$, Xiangzhi Meng ${ }^{1}$, Fushun Zhang ${ }^{1}$, Siyuan Zheng ${ }^{5,6}$, Yidong Chen ${ }^{5,6}$, Zhinan Yin ${ }^{7}$, Ross Kedl ${ }^{8}$, Booki Min ${ }^{9}$, Christopher A. Hunter $^{10}$, Yan Xiang ${ }^{1}$, Paolo Casali ${ }^{1}$ and Zhenming $\mathrm{Xu}^{1, *}$

10 Affiliations: ${ }^{1}$ Department of Microbiology, Immunology and Molecular Genetics, The Long School of Medicine, University of Texas Health Science Center San Antonio (UTHSCSA); ${ }^{2}$ The Xiangya School of Medicine; ${ }^{3}$ Department of Gastroenterology, The Third Xiangya Hospital; ${ }^{4}$ Institute of Pediatrics, The Second Xiangya Hospital, Central South University, Changsha, Hunan, China; ${ }^{5}$ Department of Population Health Sciences, The Long School of Medicine, UTHSCSA; 'Greehey Children's Cancer 15 Research Institute, UTHSCSA; ${ }^{7}$ The First Affiliated Hospital, Biomedical Translational Research Institute and Guangdong Province Key Laboratory of Molecular Immunology and Antibody Engineering, Jinan University, Guangzhou, Guangdong, China; ${ }^{8}$ Department of Immunology and Microbiology, School of Medicine, University of Colorado; ${ }^{9}$ Department of Inflammation and Immunity,

Lerner Research Institute, Cleveland Clinic Foundation; ${ }^{10}$ Department of Pathobiology, School of 20 Veterinary Medicine, University of Pennsylvania.

*Correspondence to: Zhenming Xu (xuz3@uthscsa.edu; 1-210-567-3964). 
Abstract: B cells are exposed to innate and T cell stimuli during the antibody response, although whether and how they functionally integrate such signals are unclear. Here we have identified IL-27 as the cytokine specifically produced by murine B cells upon sequential stimulation by TLR ligands and then

25 CD154 and IL-21, the hallmark factors of T follicular helper cells, and during the T-dependent antibody response to a conjugated hapten or virus infection. B-cell Il27p28 transcription is concomitant with increased locus accessibility and depends on newly induced BATF3 transcription factor. IL-27producing B cells are inefficient in antibody secretion, but cooperate with IFN $\gamma$ to promote proliferation, survival, class-switching and plasma cell differentiation of CD40-activated B cells, leading to optimal 30 IgG2a and IgG1 responses. Overall, IL-27-producing B cells function as "helper" B cells that integrate the innate and adaptive stages of the antibody response. 


\section{Main Text}

For effective antibody responses to infectious and environmental antigens, specific B cells recognize arrayed epitopes that crosslink their B cell receptors (BCRs) and later need to be engaged by CD154 (CD40 ligand, CD40L) expressed on cognate $\mathrm{T}$ helper (Th) cells for robust proliferation and differentiation in germinal centers (GCs), from which plasma cells emerge to secrete mature antibodies. These are of switched Ig isotypes (IgG, IgA and IgE) and high affinity, as underpinned by Ig locus class switch DNA recombination (CSR) and somatic hypermutation (SHM), respectively (1). Prior to the Tdependent adaptive phase, both specific and bystander B cells have already been exposed to antigen- or

40 host cell-derived stimuli that engage innate receptors, particularly Toll-like receptors (TLRs). Accordingly, engagement of B-cell TLR can synergize with BCR crosslinking to induce AID, a DNA deaminase essential for CSR and SHM (2), and elicit class-switched and high-affinity IgG antibodies during the T-independent phase of antibody responses or stand-alone T-independent responses (3). In immune-competent subjects, TLR ligands can function as adjuvants to potentiate antibody responses, 45 partially by priming dendritic cells (DCs) that in turn activate Th cells (4). However, the relevance of TLR priming to $\mathrm{B}$ cell activation by $\mathrm{T}$ cell stimuli in the T-dependent antibody response remains unclear. Independent from their differentiation into antibody-secreting plasma cells, B cells are known to produce cytokines (5-8), including those that direct CSR from IgM to specific Ig isotypes, such as IL-4 (IgG1), TGF $\beta$ (IgA) and IFN $\gamma$ (murine IgG2a), thereby supplementing cytokine production by Th cells.

50 In addition, mouse B cells can be induced to produce cytokines IL-10 and IL-35 (heterodimer of Il12aencoded IL12 $\alpha$ and Ebi3-encoded EBI3), thereby acting as immunosuppressive B regulatory (Breg) cells in several pathophysiological conditions $(9,10)$. Here, we explore new regulatory functions of B cells, starting with profiling cytokine gene induction in B cells by innate stimuli TLR ligands in combination with $\mathrm{T}$ cell stimuli, thereby mimicking $\mathrm{B}$ cell activation during the antibody response. 


\section{$55 \quad$ Results}

\section{Potent IL-27 induction in B cells}

Purified mouse naive spleen B cells expressed high levels of Il10 when stimulated by TLR4 ligand LPS (fig. S1A-S1C). Addition of membrane-bound recombinant CD154 to LPS dampened Il10 expression and specifically induced transcription of $I l 27 p 28$, but not $I l 12 a$ (Fig. 1A, 1B, fig. S1C, S1D,

Table S1, S2), leading to intracellular IL-27p28 protein expression and secretion of cytokine IL-27, which is composed of IL-27p28 and EBI3 (Fig. 1C, fig. S1E) - Ebi3, an ortholog of EBI3 identified in EBV-infected human B cells (11), was similarly upregulated. Il27p28 was also induced when other TLR9 ligands were used and in lymph node B cells, which are mature follicular B cells (fig. S1F, S1G).

While marginally affected by IL-4, a Th2 cytokine that synergizes with CD154 in activating B cells,

65 B-cell IL-27 induction was markedly enhanced by IL-21, the hallmark cytokine of T follicular helper (Tfh) cells (Fig. 1A, 1D, fig. S2A, Table S1). This resulted in $I l 27 p 28$ promoter activation (GFP expression) in $70 \%$ of B cells from $\operatorname{Tg}(I l 27 p 28-G f p)$ reporter mice and IL-27 secretion at high levels, e.g., comparable to those from LPS-activated macrophages, in which IL-27 production was not enhanced by CD154 or IL-21 (Fig. 1E, 1F, fig. S2B, S2C). Priming B cells with LPS, which upregulated surface

70 CD40 expression, followed by stimulation with CD154 and IL-21 was as effective as co-stimulation in inducing IL-27 (Fig. 1E, fig. S3A, S3B), in association with enhanced B cell survival (but not expression of selected surface markers), as compared to CD154 and IL-21-stimulated B cells (fig. S3B-S3D). Such induction by sequential innate and adaptive stimuli could be extended to priming by other TLR ligands (irrespective of their potency in inducing B cell proliferation, plasma cell differentiation and expression 75 of CD11b and CD11c) and CD40 engagement by an agonistic mAb ( $\alpha$ CD40), concomitant with robust cluster formation (Fig. 1G, fig. S4A-S4C). BCR crosslinking, by contrast, failed to prime B cells for IL27 induction (fig. S4D), showing the specific synergy of TLR and CD40 signals in this process. 


\section{B cells are important $I L-27$ producers in vivo}

Owing to their ability to induce IL-27p28 and large numbers in secondary lymphoid organs, CD19 B cells upregulated IL-27p28 levels in C57 mice immunized with alum-mixed NP-CGG together with LPS and accounted for $75 \%$ of all IL-27p28 expression (Fig. 2A, fig. S5A, S5B) - likewise, they contributed to the majority of homeostatic IL-27p28 levels, as also confirmed by highly correlating $I l 27 p 28$ and Ebi3 expression with that of $C d 79 b$ (fig. S5C). Spleen cells displaying IL-27p28 expression were mostly B220 ${ }^{+}$and localized in B cell follicles and extrafollicular areas, with a subset of such cells

85 showing concentrated signals at the plasma membrane (Fig. 2B, fig. S6A). IL-27p28 ${ }^{+}$B cells could also be elicited in draining lymph nodes and the spleen upon intranasal (i.n.) infection by vaccinia virus (the Western Reserve strain, $\mathrm{VV}_{\mathrm{WR}}$ ), whose DNA activates TLR8, but not TLR9 in DCs (12), starting at $\mathrm{d} 3$ and markedly increasing by d 7, and segregated within $I l 27 p 28$-GFP ${ }^{+}$cells in $T g(I l 27 p 28-G f p)$ reporter mice (Fig. 2C, fig. S7A, S7B) - EBI3 expression was also upregulated in Il27p28-GFP ${ }^{+}$B cells. Whether

90 induced by NP-CGG plus alum and LPS immunization or VV $\mathrm{VR}_{\mathrm{WR}}$ infection, IL-27p28 ${ }^{+} \mathrm{B}$ cells were distinct from IL-10 $10^{+}$B cells, e.g., GFP ${ }^{+}$B cells in $I l 10^{I R E S-G f p}$ mice (Fig. 2B, fig. S6B, S7C, S7D).

In $\mu \mathrm{MT}$ mice, which had virtually no B cells, expression of $I l 27 p 28$ and Ebi3 was significantly decreased, leading to reduced IL-27 secretion - expression of 1110 and $I l 12 a$ was also impaired (Fig. 2D, fig. S8A-S8C). In NP-CGG plus alum and LPS immunized $m b 1^{+/ c r e} I l 27 p 28^{f l f l}$ mice (fig. S9A), mixed

95 bone marrow chimera mice generated using $\mu \mathrm{MT}$ and $I l 27 p 28^{-/-}$mice as donors $\left(\mu \mathrm{MT}: I l 27 p 28^{-/-}\right.$, fig. S9A, S9B) or chimera mice using $\mu \mathrm{MT}$ and $E b i 3^{-/-}$mice as donors ( $\mu \mathrm{MT}: E b i 3^{-/-}$, fig. S9C), ablating Bcell $1 / 27 p 28$ or Ebi3 resulted in B cells-specific loss of IL-27p28 or EBI3 expression, reduction in their gene expression in the spleen and defective IL-27 secretion into the circulation - IL-27 levels peaked at d 14 in "wildtype" mice (Fig. 2E, 2F, fig. S9D, S9E). The higher IL-27p28 and EBI3 levels in B cells 100 that those in $\mathrm{CD}^{-} 9^{-}$cells also confirmed an important role of B cells in IL-27 production (fig. S9D). 


\section{B-cell IL-27 optimizes IgG2a responses}

In association with their defective IL-27 production, mice with B cell-specific deficiency in Il27p28 $\left(\mathrm{mbl}^{+/ c r e} / 127 \mathrm{p} 28^{\mathrm{fl} / \mathrm{fl}}\right.$ ) or $\mathrm{Ebi3}\left(\mu \mathrm{MT}: \mathrm{Ebi3}^{-/}\right.$) were impaired in mounting IgG2a responses to immunization with NP-CGG plus alum and LPS, showing reduced levels of NP-binding IgG2a, including high-affinity $105 \mathrm{NP}_{4}$-binding IgG2a at $\mathrm{d} 7$ and $\mathrm{NP}_{30}$-binding IgG2a at d 14, but largely normal levels of NP-binding IgG1, IgG2b and IgG3 (Fig. 2F, fig. S10A). Total IgG2a as well as IgM and IgG3, but not IgG1 or IgG2b, were also reduced (fig. S10B), likely reflecting a role of B-cell IL-27 in Ig responses to the CGG and LPS components in the immunogen. The defects in IgG2a antibody responses were due to impairment in CSR to IgG2a, as shown by significant reduction in germline $I \gamma 2 a-C \gamma 2 a$ and post-recombination $I \mu$ $110 C \gamma 2 a$ transcripts, the molecular marker of ongoing and completed CSR to IgG2a, respectively, and classswitched IgG2a ${ }^{+}$B cells (Fig. 2E, fig. S11A). By contrast, B cell survival and differentiation into GC B cells and plasma cells were not affected in these mice; neither was expression of AID (Fig. 2E, fig. S11B).

Murine IgG2a and human IgG3 are the IgG isotype elicited first upon viral infections $(13,14)$. IgG2a 115 has potent anti-viral activities through complement activation, engagement of activating Fcy receptors and binding of viral antigens via both $\mathrm{F}(\mathrm{ab})$ arms due to their long-hinge region for effective neutralization - by contrast, IgG1 appears later and effects neutralization by high-affinity antigen-binding via a single $\mathrm{F}(\mathrm{ab})$. Accordingly, $m b 1^{+/ c r e} I l 27 p 28^{\mathrm{fl} / \mathrm{fl}}$ and $\mu \mathrm{MT}$ :Ebi3 ${ }^{-/-}$mice produced less virus-specific IgG2a (and IgG) antibodies upon i.n. $\mathrm{VV}_{\mathrm{WR}}$ infection (Fig. 2G, fig. S10C), consistent with their reduced 120 CSR to IgG2a (Fig. 2H). This resulted in persistence of viruses in $\mu \mathrm{MT}$ :Ebi3 $3^{-/}$mice in the lung, dissemination of such viruses into the brain, and loss of more body weight (Fig. 2J, fig. S11D). Thus, IL-27-producing B cells play an important role in CSR to IgG2a and optimal IgG2a responses, including those that effect the host anti-viral immunity. 


\section{Requirement for BATF3 in IL-27+B cell generation}

Concomitant with IL-27p28 induction in B cells, the chromatin accessibility in the promoter and a proximal enhancer (Enhancer I) of the Il27p28 locus was increased, as compared to LPS-stimulated B cells, in which two upstream enhancers (Enhancer II and III) were also accessible (Fig. 3A, fig. S12A, Table S3). To identify trans-factors recruited to such differentially accessible regions (DARs), transcriptome analysis of B cells stimulated to express $I l 27 p 28$ at different levels revealed that $I l 27 p 28$ expression showed a strong positive correlation with that of Batf3, the best among all transcription factor genes and top 5 among all genes $\left(\mathrm{R}^{2}=0.799\right.$; Fig. 3B, Table S4, Table S5) - vice versa, expression of Batf3 tightly correlated with that of $I l 27 p 28$ among all cytokine and chemokine-coding genes (fig. S12B, Table S6, Table S7). Importantly, DARs in IL- $27^{+}$B cells were enriched with the motif bound by the BATF family of factors (Fig. 3C, Table S8), among which BATF3 was induced in such B cells, in 135 contrast to the constitutive Batf expression and no Batf2 expression in B cells (fig. S12C, Table S5).

$\mathrm{Batf}^{-/-}$B cells were severely impaired in $1 / 27 p 28$ transcription induction and IL-27 secretion, exemplifying an overall defect in expressing genes involved in the IFN $\gamma$ and/or anti-viral responses (Fig. 3D-3F, fig. S13A, S13B, Table S9-S11). They, on the other hand, showed heightened expression of Il10 and genes involved in myeloid cell activation/differentiation, as characteristically upregulated by LPS by BATF3 (if induced) without affecting the overall chromatin accessibility (fig. S13C). Mice with B cell-specific Batf3 deficiency ( $\mu \mathrm{MT}:$ Batf $^{-/-}$) showed decreased Il27p28 expression and IL-27 secretion, but increased Il10 expression, upon immunization with NP-CGG plus alum and LPS (Fig. 3G, 3H). They were impaired in mounting specific $\operatorname{IgG} 2 \mathrm{a}$ responses, concomitant with reduced generation of $145 \quad \mathrm{IgG}_{2} \mathrm{a}^{+} \mathrm{B}$ cells and expression of $\mathrm{I} \gamma 2 \mathrm{a}-\mathrm{C} \gamma 2 \mathrm{a}$ and $\mathrm{I} \mu-\mathrm{C} \gamma 2 \mathrm{a}$ transcripts as well as Aicda (Fig. 3G-3I, fig. S14), showing a non-redundant B cell-intrinsic role of BATF3 in IL-27 induction and IgG2a responses. 


\section{Supporting role of $\mathrm{NF}-\mathrm{KB}$ in $\mathrm{IL-27}$ production}

BATFs belong to the leucine zipper transcription factor family, which also includes the AP-1 factor c-JUN and c-FOS. With a high-affinity DNA-binding domain but no transactivation domain, they 150 modulate gene expression by competing with c-JUN or c-FOS for the same AP-1 sites to inhibit transcription or by interacting with (positive or negative) transcription factors recruited to neighboring cis-elements to strengthen the activity of such partner factors (15-17). In addition to BATF-binding sites, $\kappa \mathrm{B}$ sites were enriched in DARs in IL-27-producing B cells (Fig. 3C, Table S8). Accordingly, the Il27p28 promoter region and three enhancers have consensus $\kappa \mathrm{B}$ motifs near an $\mathrm{AP}-1$ site, with three 155 putative BATF $/ \kappa \mathrm{B}$ composite sites in Enhancer I and III (fig. S15). In B cells stimulated to induce

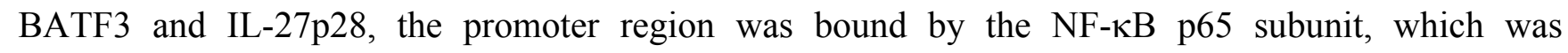
synergistically activated (as indicated by Ser536 phosphorylation, p65-S536p) by LPS and $\alpha$ CD40 (fig. S16A, S16B). In such B cells (as well as in LPS-stimulated B cells), genome-wide binding sites of NF$\kappa \mathrm{B}$ p65 were focused on promoters and proximal enhancers, as clustering around transcription start sites 160 (TSSs; fig. S16C). They were also enriched in BATF(3)-binding motifs (fig. S16D), suggesting corecruitment of NF-אB and BATF3 to the same cis-elements.

Engagement of CD40, which is a TNFR superfamily member, elicited stronger B-cell NF-кB p65 activation than TLR signals (Fig. S15B). Consistent with the dependency of B-cell CD40 (and TNFR in general) on linear ubiquitin assembly complex (LUBAC, composed of HOIL, HOIP and SHARPIN) for

165 signaling $(18,19)$, p65-S536p was reduced in $\operatorname{Sharpin}^{\text {cdmp }}$ B cells, in which a single nucleotide deletion causing frameshift and premature translation termination, resulting in no SHARPIN expression (fig. S17A). These B cells were defective in Il27p28 and Ebi3 expressing and IL-27 secretion (fig. S17B). Irrespective of their Sharpin genotype, B cells had highly concordant expression of Il27p28 and Batf3, the two most upregulated genes by IL-27-inducing stimuli (Fig. 3K, fig. S17C, S17D). 
As the target of B cell-produced IL-27, IL-27R $\alpha$, which is the IL-27-specific subunit of the receptor, was highly expressed in B cells - the other subunit GP130 is shared by receptors of IL-6 family cytokines (fig. S18A, S18B). In $C d 19^{+/ c r e} I l 27 r a^{f l f l}$ mice and mixed bone marrow chimera $\mu \mathrm{MT}: I l 27 r a^{-/-}$mice, specific IgG2a response to immunization with NP-CGG plus alum and LPS was reduced (Fig. 4A, fig. 175 S18C-S18E, fig. S19A, S19B), in agreement with previous studies (20). Accordingly, CSR to IgG2a was impaired in $\mu \mathrm{MT}: I l 27 \mathrm{ra}^{-/-}$mice (fig. S19C-S19G). Upon VV $\mathrm{WR}_{\mathrm{WR}}$ infection, $\mu \mathrm{MT}: I l 27 \mathrm{ra}^{-/-}$mice produced less virus-specific IgG2a and failed to eradicate virus in the lung or limit virus spreading, consistent with their reduced serum virus-neutralization activities (Fig. 4B-4F).

While significantly reduced (by $60 \%$ ) upon ablation of $\mathrm{IL}-27^{+} \mathrm{B}$ cells or $1 / 27 \mathrm{ra}$-expressing B cells as 180 their potential target cells, IgG2a responses, as well as CSR to IgG2a, were abrogated in mice with B cell-specific compound deficiency in Il27ra and Ifngrl ( $\mu \mathrm{MT}$ :Il27ra ${ }^{-/}$Ifngrl ${ }^{-/}$; Fig. 4G, 4H, fig. S20A). The complementary roles of B-cell IL-27 and IFN $\gamma$ receptor signals would provide a parsimonious explanation for IgG2a elimination in whole-body knockout (KO) $I l 27 \mathrm{ra}^{-/}$Ifngrl ${ }^{-/-}$mice (fig. S20B). They, together with possible reciprocal induction of IL-27 and IFN $\gamma$ in all cell sources, would also explain 185 the $90 \%$ reduction in IgG2a levels in whole-body single $\mathrm{KO} \mathrm{Il2}_{\mathrm{ra}}^{-/}$and $\mathrm{Ifngrl}^{-/}$mice (fig. S20B), as compared to the $60 \%$ reduction in B cell-specific single $\mathrm{KO} \mu \mathrm{MT}: I l 27 \mathrm{ra}^{-/}$and $\mu \mathrm{MT}: I f n g r 1^{-/-}$mice.

Unexpectedly, $\mu \mathrm{MT}: I l 27 \mathrm{ra}^{-/}$Ifngr $I^{-/-}$mice were defective in the IgG1 response to immunization, concomitant with impairment in the generation of $\mathrm{GL}-7^{+} \mathrm{FAS}^{+} \mathrm{GC}$ B cells and $\mathrm{CD} 138^{+}$plasma cells as well as Tfh cells (Fig. 4G, 4H, fig. S20A). Accordingly, whole-body KO Il27ra $\mathrm{rfngrl}^{-/-}$mice displayed reduced homeostatic IgG1 levels, in addition to the abolished IgG2a levels, and early mortality

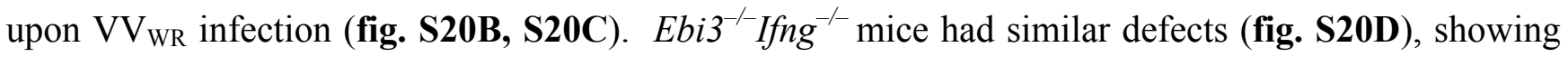
the synergy of IL-27 and IFN $\gamma$ in effecting anti-viral responses by B cells and CD $8^{+} \mathrm{T}$ cells $(21,22)$. 


\section{IL-27 and IFNy receptor signals modulate $B$ cell differentiation}

Consistent with the synergy their respective receptor signals in B cells in vivo, IL-27 and IFN $\gamma$ 195 synergized to activate STAT1, but did not activate any other STATs analyzed, in CD154-primed B cells (fig. S21A). These cells could also be conditioned by IL-21 to display more STAT1 activation in response to IL-27 or IFN $\gamma$, which together, however, abrogated activation of STAT3 by IL-21 (fig. S21A). Such divergent activation of different STATs was associated with differential regulation of many gene sets, as exemplified by IL-27 and IFN $\gamma$ upregulation of "Type 1" genes (particularly Tbx21, Cxcr3, $200 C x c l 9$ and $C x c l 10)$ and germline $\mathrm{I} \gamma 2 \mathrm{a}-\mathrm{C} \gamma 2 \mathrm{a}$ transcription while downregulating germline I $\gamma 1-\mathrm{C} \gamma 1$ transcription and Aicda expression (Fig. 5A, 5B, fig. S21B, Table S13, S14). IL-27 enhanced plasma cell differentiation in B cells stimulated with CD154 plus IL-21 and, together with IFN $\gamma$, rescued such B cells from the killing effect of IL-21 (Fig. 5C, fig. S21C, S21D). Nevertheless, IL-27 and IFN $\gamma$ failed to direct CSR to IgG2a in B cells stimulated with CD154 or CD154 plus IL-21, consistent with lack of 205 robust Aicda expression in either case (Fig. 5D, fig. S21B).

In contrast to T-dependent "primary" stimulus CD154 (1), alone or with IL-21, the T-independent primary stimulus LPS programmed B cells to undergo CSR to IgG2a and concomitant reduction of CSR to IgG3 in response to IL-27 or IFN $\gamma$ despite effect of IFN $\gamma$ in killing TLR-activated B cells, with little impact on plasma cell differentiation (Fig. 5C, 5D, fig. S21C, S21E). These effects were associated with 210 IL-27 and IFN $\gamma$ activation of STAT1 and their additive effect in STAT3 activation - with IL-27 and IFN $\gamma$ also synergized with IL-4 to induce moderate levels of STAT5 activation (fig. S21A). They were likely underpinned by the induction of Aicda and germline I $\gamma 2 \mathrm{a}-\mathrm{C} \gamma 2 \mathrm{a}$ transcription together with $T b \times 21$ and other Type 1 genes (Fig. 5A, fig. S21B, Table S15). Finally, IL-27, but not IL-35, antagonized the effect of IL-4 in LPS-stimulated B cells by downregulating induction of Type 2 gene expression and CSR to 215 IgG1 without affecting B cell proliferation or survival (fig. S22, Table S16, S17). 


\section{$I L-27^{+} B$ cells synergize with IFN $\gamma$ to induce CSR to IgG2a}

Owing to their production of IL-27 that inhibited CSR to non-IgG2a isotypes, LPS and CD154stimulated B cells underwent little CSR to IgG2b, IgG3 or - in the presence of IL-4 or TGF $\beta$ - IgG1, IgE or IgA (Fig. 5E, 5F, fig. S23A-S23C). IL-27 ${ }^{+}$B cells were also refractory to plasma cell differentiation and IgM secretion, consistent with reduced expression of $\operatorname{Prdm} 1$, which encodes BLIMP1, the master transcription factor of plasma cell differentiation (fig. S23D-S23F). Batf3 and Sharpin, while important for IL-27 induction, were dispensable for CSR and plasma cell differentiation (fig. S24).

B cells stimulated to produce IL-27 failed to switch to IgG2a, but could to do so in the presence of IFN $\gamma$ (Fig. 5E, fig. S25A, S25B). They upregulated selected adhesion molecules and formed doublets 225 with LPS-stimulated B cells (Fig. 5G, fig. S25C, Table S18). They also induced IgG2a switching and secretion in target $\mathrm{B}$ cells activated by LPS, and remained $\mathrm{IgG} 2 \mathrm{a}^{-\mathrm{ve}}$ per se (Fig. 5H, fig. S25D). In target B cells activated by CD154 or $\alpha \mathrm{CD} 40$ (alone or with IL-21), IL-27 ${ }^{+}$B cells - unlike recombinant IL-27 - induced CSR to IgG2a (Fig. 5I, fig. S26A). By contrast, "control” B cells stimulated with LPS was inefficient in inducing IgG2a in target B cells (fig. S26B). In addition, IL-27 ${ }^{+} \mathrm{B}$ cells and IFN $\gamma$ together enhanced survival and proliferation of target B cells activated by CD154 and IL-21 (fig. S26C).

The ability of IL-27 ${ }^{+}$B cells to direct T-dependent CSR to IgG2a was synergistically enhanced by IFN $\gamma$ (but not IL-4), leading to switching in $25 \%$ and $50 \%$ of target B cells activated by CD154 and aCD40, respectively, plus IL-21 in a manner dependent on IL-27p28 and EBI3 (Fig. 5I, 5J, fig. S26A). Such a synergy was independent of the boost of target B cells proliferation, as the increase was across 235 cell divisions, with early divisions also displaying high IgG2a levels (fig. S26B). Finally, the synergistic IgG2a induction was maximized (over 60\%) when target B cells were activated by LPS plus CD154 and IL-21, the same stimuli that also induced IL-27 $7^{+}$B cells (Fig. 5I, fig. S26), showing that IL-27 "regulatory" B cells could be induced to differentiate into IgG2a-expressing “effector" B cells in trans. 


\section{Discussion}

Here, we have reported the identification of IL-27-producing B cells, their induction and function in the T-dependent antibody response, and underlying mechanisms. These include the pivot role of BATF3 in controlling the B cell cytokine production program and a new paradigm of induction of CSR to IgG2a, the IgG isotype immensely relevant to the host defense against viral infections.

Since its discovery (23), IL-27 has been thought to be produced mainly by monocytes. As unveiled 245 here, IL-27 $7^{+}$B cells could be robustly induced by a unique condition, i.e., priming by TLR signals (which can likely be substituted by other innate signals) and then exposure to adaptive CD40 signals, and potentiated proliferation, survival and differentiation of target $\mathrm{B}$ cells in response to $\mathrm{T}$ cell stimuli, thereby bridging the innate phase and adaptive phase of the T-dependent IgG2a and IgG1 responses. TLR ligands, whether co-encapsulated with antigens in nanoparticles or co-delivered with them as separate entities $(4,24)$, enhance IgG2a and IgG1 responses by acting directly on B cells (and DCs). They would allow B cells to induce AID/CSR upon BCR engagement to effect T-independent antibody responses or readily produce IL-27 upon engagement by Tfh cells to boost T-dependent responses. Absent such adaptive signals from BCR or Tfh cells, TLR-primed B cells may secrete IL-10, IL-6 or even IL-35, thereby inhibiting the antibody response, as previously suggested (10). In TLR-licensed B 255 cells, BATF3, upon induction by Tfh cell factors, skewed the B cell cytokine program in favor of IL-27 and away from IL-10. Such opposing effects could be underpinned by the activities of BATF3 as both a transcription activator and inhibitor, as previously shown also in $\mathrm{CD} 4^{+} \mathrm{T}$ cells (25). The marginal impact of BATF3 on CSR highlights its difference and BATF, which - like EBI3 - was originally identified in EBV-transformed human B cells (26) and promotes CSR (27), but is partially redundant at best with 260 BATF3 in IL-27 induction. Likewise, the role of SHARPIN in NF- $\mathrm{B}$ activation for IL-27 induction, but not CSR, contrasts the role of RAB7 in promoting NF- $\mathrm{BB}$ activation for CSR induction (28). 
TLR priming would allow both antigen-specific and bystander B cells to receive Tfh cell help, most likely by upregulating CD40 expression. This can tighten the CD40:CD154 engagement and sustain the “entanglement" between B cells and Tfh cells, thereby strengthening the MHC II:TCR interaction 265 involving cognate B cells or making up for it in bystander B cells - upregulated surface CD40 would be internalized upon engagement by CD154 (28). IL-27 $7^{+} \mathrm{B}$ cells, despite losing the ability in secreting antibodies, produced IL-27 that may in turn act on Tfh cells to upregulate IL-21 (29), consistent with a putative B cell function in governing Tfh cell dynamics in GCs, in addition to providing ICOSL (30). This also raises the possibility of a positive feedback loop involving IL- $21^{+}$Tfh cells and IL- $27^{+} \mathrm{B}$ cells to nearly synchronize IL-21 and IL-27 production for the antibody response to viral infections, after an earlier DC-produced IL-27 peak that would mobilize $\mathrm{CD}^{+} \mathrm{T}$ cells for rapid anti-viral responses - IL-27 may also be dependent on BATF3 may also mediate production of IL-27 in DCs, within which it is recruited to Enhancer II and III in the Il27p28 locus (ChIP-Seq data from Ref. (31)).

As helper cells and likely distinct from age-associated B cells or T-bet ${ }^{+}$B cells (32), IL-27 $7^{+}$B cells, 275 through secreted IL-27 and likely also other elements, such as adhesion molecules involved in "synapsis", promoted the growth of target B cells activated by T-dependent stimuli and their differentiation into effector plasma cells that produced large amounts of $\operatorname{IgG} 2 \mathrm{a}$ and $\operatorname{IgG} 1$ antibodies. Such enhancement effects were reciprocally reinforced, complemented and/or backed up by those of a powerful collaborator in IFN $\gamma$, most notably CSR to IgG2a at a high level (up to 50\%), which rivals the high efficiency of CSR 280 to IgG1 induction. IFN $\gamma$ may be derived from $\mathrm{Th} 1 \mathrm{CD} 4^{+} \mathrm{T}$ cells or type $1 \mathrm{Tfh}$ cells, leading to a cytokine milieu rich in IL-21, IL-27 and IFN $\gamma$ and essential for CSR to IgG2a - within this milieu, even IL-27 $\mathrm{B}$ cells can potentially differentiate into $\mathrm{IgG}_{2} \mathrm{a}^{+} \mathrm{B}$ cells. IL- $27^{+} \mathrm{B}$ cells may be engaged in tri-cellular interactions with CD40-activated target B cells and IL- $21^{+} \mathrm{IFN} \gamma^{+} \mathrm{Tfh}$ cells to strengthen the GC response, consistent with an important role of IFN $\gamma$ (and STAT1) in antibody and autoantibody responses (33, 34). 
285 The importance of such cellular interactions is also emphasized by the inability of recombinant IL-27 and IFN $\gamma$ in the induction of CSR to IgG2a. Finally, IL-27 and IFN $\gamma$ collaborate to mediate host responses to viral infections and likely the generation and function of Tregs and ILCs (35-37).

The effect of IL-27 (as produced by BATF3 ${ }^{+} \mathrm{B}$ cells in vivo) and IFN $\gamma$ in optimizing the response of their target B cells to T cell stimuli is likely underpinned by the unique pattern of STAT signal outputs, 290 with IL-21 playing a key role. IL-21 is critical for GC B cell as well as Prdm1 induction and plasma cell differentiation in a STAT3-dependent manner $(38,39)$, but promotes B cell death, as previously reported and re-emphasized here (40). While IL-27 or IFN $\gamma$-mediated STAT1 activation was enhanced by IL-21, IL-21-activated STAT3 is synergistically abrogated by IL-27 and IFN $\gamma$. The different effects on STAT activation would ultimately differentially affect the target genes of IL-27 and IFN $\gamma$. The impact of 295 abrogated STAT3 activation on Prdm 1 expression and plasma cell differentiation may be mitigated by STAT1 and other transcription factors. As AID promotes GC B cell death by generating double-strand DNA breaks (DSBs) that, if overwhelming the DNA repair machinery, can instigate apoptosis $(41,42)$, downregulation of this genotoxic enzyme by IL-27 and IFN $\gamma$ likely contribute to their pro-survival effect on target B cells, as assisted by the upregulation of the anti-apoptotic genes. That IL-4 and IL-21 together trigger massive death of CD154-stimulated B cells suggests that these two cytokines do not function on the same B cell in vivo without strong B-cell survival factors (such as BAFF or combined IL-27 and IFN $\gamma$ ), consistent with their different induction kinetics and the notion that most CSR to IgG1 completes before the GC reaction initiates (43-45). Nevertheless, plasma cell differentiation of $\operatorname{IgG} 1^{+} \mathrm{B}$ cells would still dependent on the full-blown GC reaction and be defective in $\mu \mathrm{MT}: I l 27 \mathrm{ra}^{-/}$Ifngr ${ }^{-/-}$mice. Overall, 305 IL-27 ${ }^{+}$B cells, together with IFN $\gamma$, coordinate innate and adaptive immune elements, either on the cell surface or secreted, to regulate antibody responses and likely diverse pathophysiological conditions owing to pleiotropic functions of IL-27 as both a pro-inflammatory and anti-inflammatory cytokine (46). 


\section{References and Notes}

310 1. Z. Xu et al. Nat. Rev. Immunol. 12, 517-531 (2012).

2. E. J. Pone et al. Nat. Commun. 3, 767 (2012).

3. M. Akkaya et al. Nat. Immunol. 19, 255-266 (2018).

4. S. P. Kasturi et al. Nature 470, 543-547 (2011).

5. W. Wojciechowski et al. Immunity 30, 421-433 (2009).

315 6. T. F. Tedder. J. Immunol. 194, 1395-1401 (2015).

7. P. Shen et al. Nat. Rev. Immunol. 15, 441-451 (2015).

8. E. C. Rosser et al. Immunity 42, 607-612 (2015).

9. A. Yoshizaki et al. Nature 491, 264-268 (2012).

10. P. Shen et al. Nature 507, 366-370 (2014).

320 11. O. Devergne et al. J. Virol. 70, 1143-1153 (1996).

12. J. Martinez et al. Proc. Natl. Acad. Sci. U.S.A. 107, 6442-6447 (2010).

13. J. P. Coutelier et al. J. Exp. Med. 165, 64-69 (1987).

14. A. Ferrante et al. Pediatr. Infect. Dis. J. 9, S16-S24 (1990).

15. K. Hildner et al. Science 322, 1097-1100 (2008).

325 16. B. C. Betz et al. J. Exp. Med. 207, 933-942 (2010).

17. T. L. Murphy et al. Nat. Rev. Immunol. 13, 499-509 (2013).

18. F. Tokunaga et al. Nature 471, 633-636 (2011).

19. F. Ikeda et al. Nature 471, 637-641 (2011).

20. D. Vijayan et al. J. Immunol. 197, 3008-3017 (2016).

330 21. J. Goulding et al. J. Immunol. 189, 2432-2440 (2012).

22. N. D. Pennock et al. Proc. Nat. Acad. Sci. U.S.A. 111, 16472-16477 (2014).

23. S. Pflanz et al. Immunity 16, 779-790 (2002).

24. B. Hou et al. Immunity 34, 375-384 (2011).

25. A. Iwata et al. Nat. Immunol. 18, 563-572 (2017).

335 26. M. J. Dorsey et al. Oncogene 11, 2255-2265 (1995).

27. W. Ise et al. Nat. Immunol. 12, 536-543 (2011).

28. H. Yan et al. J. Immunol. 204, 1146-1157 (2020).

29. M. Batten et al. J. Exp. Med. 207, 2895-2906 (2010).

30. D. Liu et al. Nature 517, 214-218 (2015).

340 31. G. E. Grajales-Reyes et al. Nat. Immunol. 16, 708-717 (2015).

32. M. S. Naradikian et al. J. Immunol. 197, 1023-1028 (2016).

33. S. W. Jackson et al. J. Exp. Med. 213, 733-750 (2016).

34. P. P. Domeier et al. J. Exp. Med. 213, 715-732 (2016).

35. A. O. Hall et al. Immunity 37, 511-523 (2012).

345 36. K. Moro et al. Nat. Immunol. 17, 76-86 (2016).

37. T. H. Nguyen et al. J. Immunol. 200, 237-247 (2018).

38. K. Ozaki et al. Science 298, 1630-1634 (2002).

39. H. Kwon et al. Immunity 31, 941-952 (2009).

40. H. Jin et al. J. Immunol. 173, 657-665 (2004).

350 41. R. T. Phan et al. Nature 432, 635-639 (2004).

42. A. Zaheen et al. Blood 114, 547-554 (2009).

43. J. S. Weinstein et al. Nat. Immunol. 17, 1197-1205 (2016).

44. M. Gaya et al. Cell 172, 517-533 e520 (2018).

45. J. A. Roco et al. Immunity 51, 337-350 e337 (2019).

46. H. Yoshida et al. Ann. Rev. Immunol. 33, 417-443 (2015). 
Acknowledgments: We wish to thank: Dr. Zhijie Liu for providing anti-NF-кB p65 rabbit polyclonal $\mathrm{Ab}$, Dr. Elizabeth Leadbetter for $I l 10^{I R E S-G f p}$ mice, Dr. William Kaiser for Sharpin ${ }^{c p d m / c p d m}$ mice, and Mr. Daniel Chupp for technical help (UTHSCSA); and Dr. Joseph Craft for advice (Yale).

Funding: supported by NIH AI 135599, AI131034, AI124172, DOD BC170448 (Z.X.), NIH AI105813, AI079705, AI138994 (P.C.), NIH AI079217, AI133589 (Y.X.) and CPRIT RR170055 (S.Z.). The UTHSCSA Flow Cytometry Core facility is supported by NIH P30 CA054174, and Genome Sequencing Facility supported by NIH P30 CA054174, S10 OD021805, and CPRIT Core RP160732 grants. Author contributions: Conceptualization(H.Y., R.W. and Z.X.), investigation (H.Y., R.W., J.W., S.W., 365 M.F., C.E.R., C.C., J.B.M., X.-D. L., N.Z., X.M., F.Z. and Z.X.), resources (R.K., B.M., C.A.H. and Y.X.), visualization (H.Z., S.Z. and Y.C.), funding acquisition (P.C. and Z.X.) and supervision (Z.X.).

Competing interests: Authors declare no competing interests. Data and materials availability: All materials are available upon request, pending material transfer agreements. Deep-sequencing data (RNASeq, ATAC-Seq and ChIP-Seq) are being deposited to the NCBI. All data, code, and materials are 370 available to any researcher for purposes of reproducing or extending the analysis.

\section{Supplementary Materials:}

Materials and Methods

Figures S1-S26

Tables S1-S21

375 Supplementary References and Notes (47-49) 


\section{Figure Legends}

Fig. 1. Induction of IL-27 in B cells. (A,B) Heatmap and Venn diagram of 127 differentially expressed genes (DEGs) in B cells after stimulation with LPS plus CD154 or LPS plus CD154 and IL-21, but not LPS or CD154, for $24 \mathrm{~h}$. Data are from two independent stimulation and RNA-Seq experiments. Genes expressed in at least one sample were analyzed. (C) Intracellular staining of IL-27p28 in CD19 ${ }^{-}$and $\mathrm{CD}_{1} 9^{+}$cells after total spleen cells were stimulated for $48 \mathrm{~h}$. (D) qRT-PCR analysis of $I l 27 p 28$ and Ebi3 expression in purified B cells stimulated, as indicated. Data were normalized to $C d 79 \mathrm{~b}$ expression and expressed as the ratio to values of freshly isolated B cells $(0$ h). (E) Flow cytometry analysis of GFP 385 expression in purified $\operatorname{Tg}(I l 27 p 28-G f p)$ B cells co-stimulated or sequentially stimulated, as the readout of transcription activity in the $I l 27 p 28$ locus. Representative of three independent experiments. (F) ELISA of IL-27 secreted into the supernatants of B cells after stimulation for $48 \mathrm{~h}$. (G) Percentages of $\mathrm{GFP}^{+}$cells by flow cytometry after purified $\operatorname{Tg}(I l 27 p 28-G f p)$ B cells were primed with a TLR ligand, as indicated, for $24 \mathrm{~h}$ and then stimulated with $\alpha \mathrm{CD} 40$ and IL-21 for $24 \mathrm{~h}$.

390 Fig. 2. Induction and function of IL-27-producing B cells in vivo. (A) Intracellular staining of IL27 p28 in CD19 ${ }^{+}$cells in C57 mice before and after immunization, as indicated. Representative of three independent experiments. (B) Flow imaging analysis of B220, IL-27p28 and GFP (indicating Ill0 transcription) in immunized $I l 10^{I R E S-G f p}$ mice (representative of three mice in two independent experiments). (C) Flow cytometry analysis of IL-27p28 protein and GFP expression to quantify IL27 p28 expression and $I l 27 p 28$ gene transcription in the spleen and mediastinal lymph node B cells from $\operatorname{Tg}(I l 27 p 28-G f p)$ mice after virus infection. (D) qRT-PCR analysis of transcript levels of cytokine genes, as indicated, in the spleen of C57 and $\mu \mathrm{MT}$ mice $7 \mathrm{~d}$ after immunization. Data were normalized to Gapdh expression. (E) qRT-PCR analysis of cytokine gene expression and CSR-related transcripts in the spleen of $m b 1^{c r e} I l 27 p 28^{f l f l}$ and $m b 1^{c r e} I l 27 p 28^{+/ f l}$ mice after immunization. Data were normalized to $C d 79 b$ expression and expressed as the fold of values in $m b 1^{c r e} I l 27 p 28^{+/ f l}$ mice (mean and s.d.). (F) ELISA of 
IL-27 production in the spleen (left panel) and NP-binding IgG2a in the circulation (right panel) in $m b 1^{\text {cre }} I l 27 p 28^{f l f l}, \mu \mathrm{MT}: E b i 3^{-/-}$and their respective "wildtype" mouse counterparts, as indicated, after immunization. (G) ELISA of virus-specific IgG2a in the circulation in $m b 1^{\text {cre }} I l 27 p 28^{f l / f l}, \mu \mathrm{MT}: I l 27 p 28^{-}$ ${ }^{\text {/- }}, \mu \mathrm{MT}: E_{b i 3^{-/}}$and their respective "wildtype" mouse counterparts $10 \mathrm{~d}$ after $\mathrm{VV}_{\mathrm{WR}}$ infection. (H) 405 Quantification of $\operatorname{IgG} 2 \mathrm{a}^{+}$B cells and CD $138^{\text {hi }}$ plasma cells/plasmablasts in mediastinal lymph nodes and the spleen by flow cytometry in $\mathrm{VV}_{\mathrm{WR}}-$ infected $m b 1^{\text {cre }} I l 27 p 28^{\text {fllfl }}$ and $m b 1^{\text {cre }} I l 27 p 28^{+/ f l}$ mice. Quantification of $\mathrm{VV}_{\mathrm{WR}}$ titers in different organs in $\mathrm{VV}_{\mathrm{WR}}-$ infected $\mu \mathrm{MT}:$ Ebi3 $^{+/+}$and $\mu \mathrm{MT}:$ Ebi3 $^{-/-}$mice. Fig. 3. BATF3 mediates in IL-27 induction in B cells. (A) ATAC-Seq analysis of the chromatin accessibility of the Il27p28 locus in stimulated B cells. (B) RNA-Seq and correlation analysis of the 410 expression level of $I l 27 p 28$ and that of 441 expressed transcription factors in duplicate B cells stimulated in vitro with nil (0 h), (CSR-inducing) LPS, CD154 and CD154 plus IL-4, as well as (IL-27-inducing) LPS plus CD154, CD154 and IL-21, and LPS plus CD154 and IL-4, for $24 \mathrm{~h}$. (C) Venn diagram depiction of DARs in B cells stimulated with LPS plus CD154 and IL-21, as compared to those in LPSstimulated B cells (left) and motifs enriched in such DARs (right). (D) Heatmap of cytokine-encoding 415 genes differentially expressed in stimulated Batf $^{+/+}$and $B a t f 3^{-/}$B cells. (E,F) qRT-PCR analysis of gene expression in $\mathrm{Batf}^{+/+}$and $\mathrm{Batf3}^{-/-} \mathrm{B}$ cells stimulated for $48 \mathrm{~h}(\mathbf{E})$ and ELISA of IL-27 secretion by these cells (F). (G-I) qRT-PCR analysis of gene expression $(\mathbf{G})$, ELISA of IL-27 in the spleen $(\mathbf{H})$ and flow cytometry analysis of switched $\operatorname{IgG}_{2} \mathrm{a}^{+} \mathrm{B}$ cells (I) in $\mu \mathrm{MT}: B a t f 3^{+/+}$and $\mu \mathrm{MT}:$ Batf $^{-/-}$mice immunized with NP-CGG plus alum with LPS for $12 \mathrm{~d}$. (K) Correlation analysis of Il27p28 expression 420 with that of Batf3 or Batf in $\operatorname{Sharpin}^{+/+}$and Sharpin ${ }^{\text {cpdm/cpdm }}$ B cells stimulated to induce CSR or IL-27 production, as indicated (Batf2 was not expressed in B cells in any condition).

Fig. 4. Function of IL-27 receptor signals in B cells. (A) ELISA of NP-binding IgM and IgG isotypes in $\mu \mathrm{MT}: I l 27 r \alpha^{-/-}$and $\mu \mathrm{MT}: I l 27 r \alpha^{+/+}$mice immunized with NP-CGG plus alum with LPS. (B-F) ELISA of virus specific IgG2a and IgG1 in serum samples in $\mathrm{VV}_{\mathrm{WR}}$-infected $\mu \mathrm{MT}: I l 27 r \alpha^{-/-}$and $\mu \mathrm{MT}: I l 27 r \alpha^{+/+}$ 
425 mice (B), the virus-neutralizing activity of serum samples (C), virus titers in organs (D), and lung histopathology $(\mathbf{E})$ and scores $(\mathbf{F})$. (G,H) ELISA of NP-binding IgG isotypes and FACS analysis of B cells in mixed bone marrow chimera mice with B cell-specific double and single KO of Il27ra and Ifngrl, as indicated, after immunization with NP-CGG, alum and LPS.

Fig. 5. Synergy of $\mathrm{IL}-27^{+} \mathrm{B}$ cells and IFN $\gamma$ on target $\mathrm{B}$ cell differentiation. (A) Venn diagram 430 depiction of DEGs induced by IL-27 and IFN $\gamma$ in B cells stimulated with CD154 for $24 \mathrm{~h}$ (top) or DEGs induced by IL-27 in B cells stimulated with CD154 or LPS for $24 \mathrm{~h}$ (bottom). (B) GSEA analysis of DEGs upregulated (top) or downregulated (bottom) by IL-27 and IFN $\gamma$ in B cells stimulated with CD154 for $24 \mathrm{~h}$. (C,D) Flow cytometry analysis of plasma cell differentiation (C) and CSR to IgG2a (D) in B cells stimulated, as indicated, for 96 h. (E) Flow cytometry analysis of CSR to different Ig isotypes in B 435 cells stimulated, as indicated, for $96 \mathrm{~h}$. (F) Flow cytometry analysis of CSR to IgG1 in $\mathrm{Il}_{27 \mathrm{ra}^{+/+}}$and $\mathrm{Il}_{27 \mathrm{ra}^{-/}} \mathrm{B}$ cells stimulated, as indicated, for $96 \mathrm{~h}$. (G) Heatmap of adhesion molecule-encoding genes upregulated in IL-27 $7^{+}$B cells (top two rows), as compared to B cells stimulated with CD154 plus nil or IL-4 (bottom rows). (H) ELISA of IgM, IgG2a and IgG3 secreted by $\mathrm{Aicda}^{-/}, \mathrm{C} 57$ and $\mathrm{Il27ra}{ }^{-/-} \mathrm{B}$ cells, as target B cells, stimulated with LPS for $96 \mathrm{~h}$ in the presence of cytokine IL-27 or IFN $\gamma$ (top 2 clusters), 440 or IL-27 $7^{+}$B cells (bottom cluster), as generated from B cells of different genotypes (as indicated) and then co-cultured with target B cells for $72 \mathrm{~h}$. (I) FACS analysis of CD45.2 ${ }^{+}$target B cells labeled with CellTrace ${ }^{\mathrm{TM}}$ (to track proliferation) and activated, as indicated, $16 \mathrm{~h}$ before co-cultured for $72 \mathrm{~h}$ with nil, $\mathrm{CD} 45.1^{+} \mathrm{IL}-27^{+} \mathrm{B}$ cells, IFN $\gamma$ or both $\mathrm{CD} 45.1^{+} \mathrm{IL}-27^{+} \mathrm{B}$ cells and IFN $\gamma$, as indicated ( $\mathrm{n}=3$ ). (J) FACS analysis in CD $45.1^{+}$target B cells, as indicated, $16 \mathrm{~h}$ before co-cultured with CD $45.2^{+} \mathrm{B}$ cells of different 445 genotypes that had been stimulated with LPS plus $\alpha$ CD40 and IL-21 for $36 \mathrm{~h}$ as inducing B cells plus IFN $\gamma$. Representative of two independent experiments. 
$A-B$

LPS+CD154 LPS+CD154+IL21

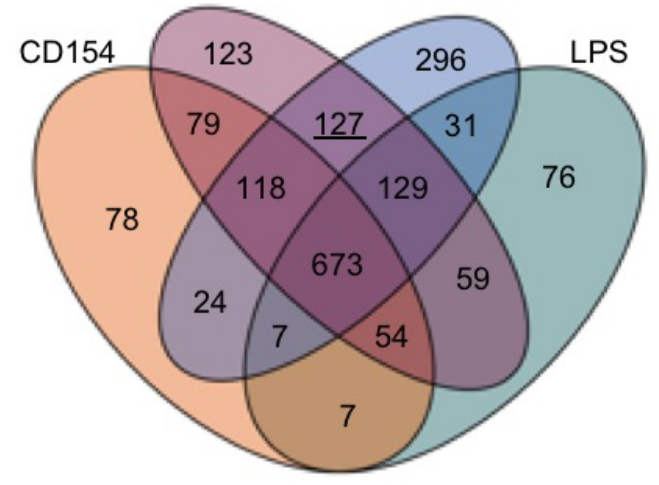

C
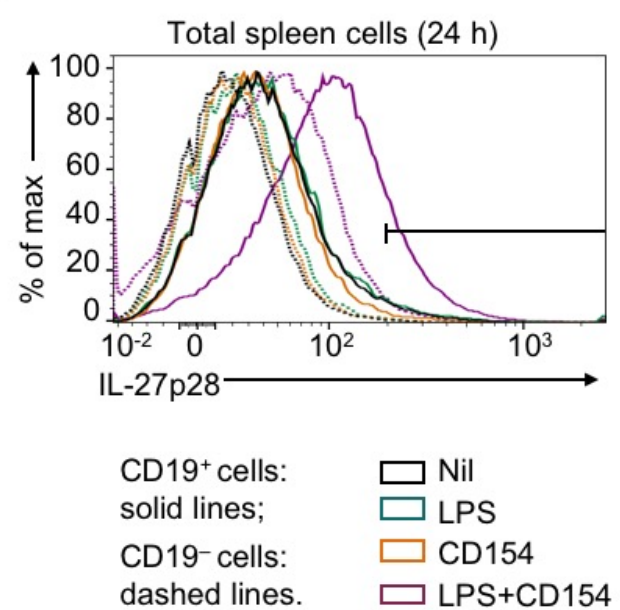

dashed lines. $\square$ LPS+CD154

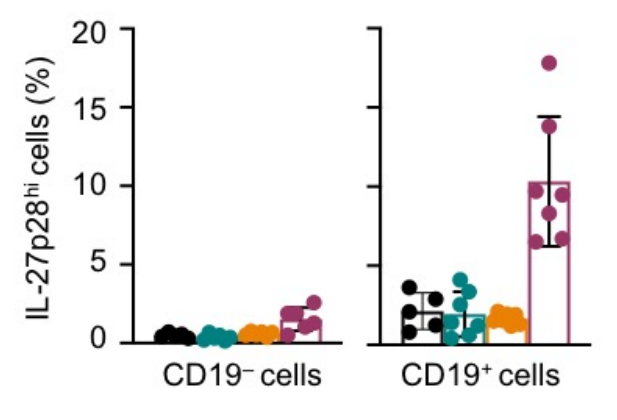

CD154+IL-21- re-
IL-27 in supernatants ( $\mathrm{ng} / 10^{6}$ cells)

$\begin{array}{lllll}0 & 0.6 & 1.2 & 1.8 & 2.4\end{array}$

$\begin{array}{lllllll}0 & 10 & 20 & 30 & 40 & 50 & 60\end{array}$

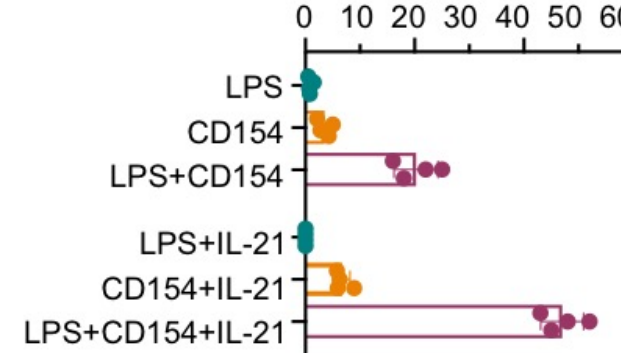

Ebi3 expression (relative) $\begin{array}{llllll}0 & 20 & 40 & 60 & 80 & 100\end{array}$ LPS CD154 LPS + CD154

LPS+IL-21 LPS+CD154+IL-21-

E

LPS

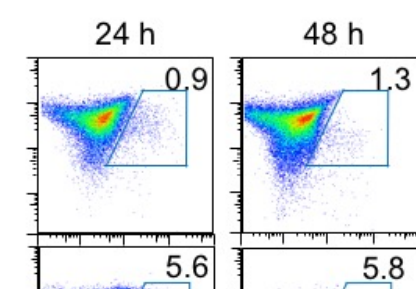

CD154+IL-21
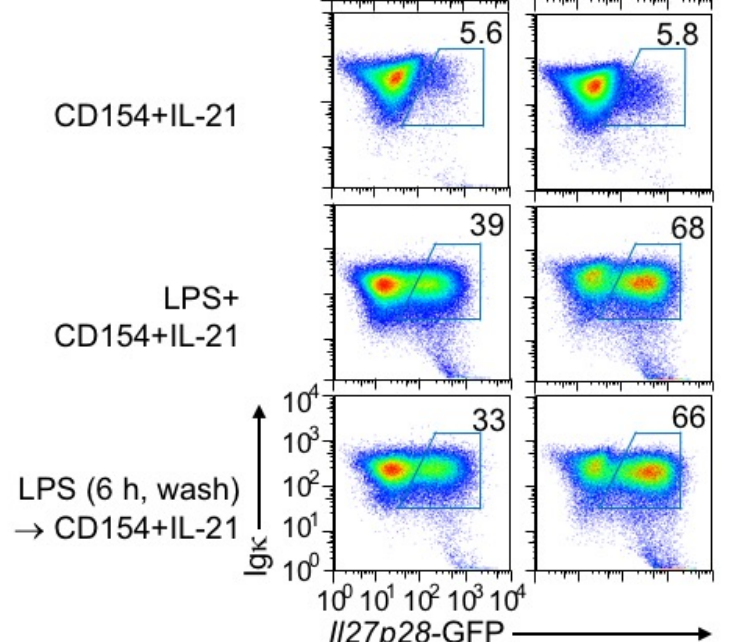

C57 B cells

C57 B cells

CD154+IL-21
LPS+CD154+IL-21

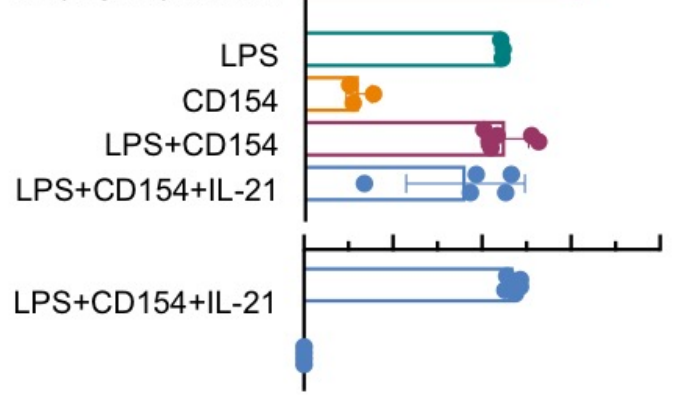

C57 bone

marrow-derived macrophages

II27p28 $8^{+/+} \mathrm{B}$ cells II27p28-- B cells

G

h 24 h 48 h GFP+ II27p28-Gfp B cells (\%)

$\longrightarrow \quad 01020304050 \quad 60$

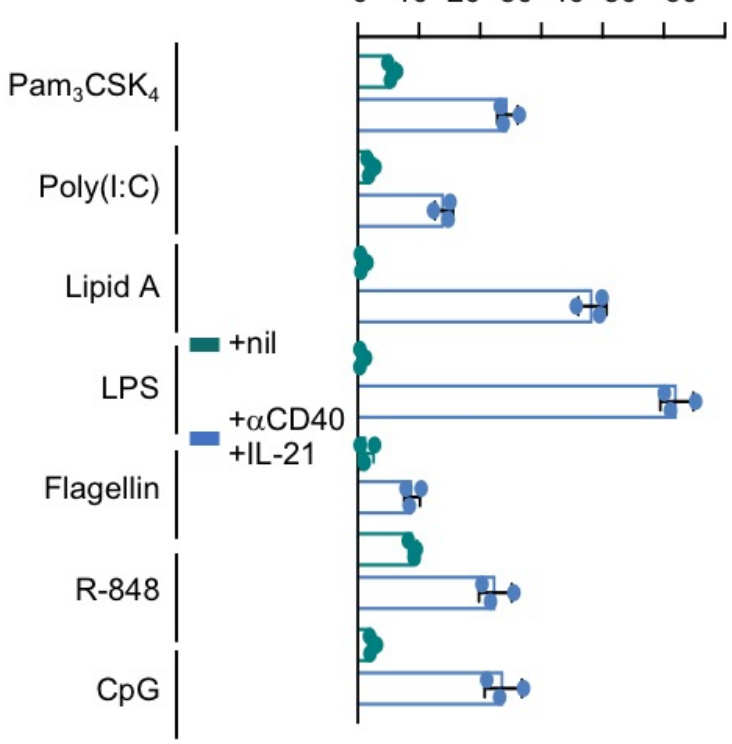


A

C57 mice (NP-CGG/alum/LPS, spleen)
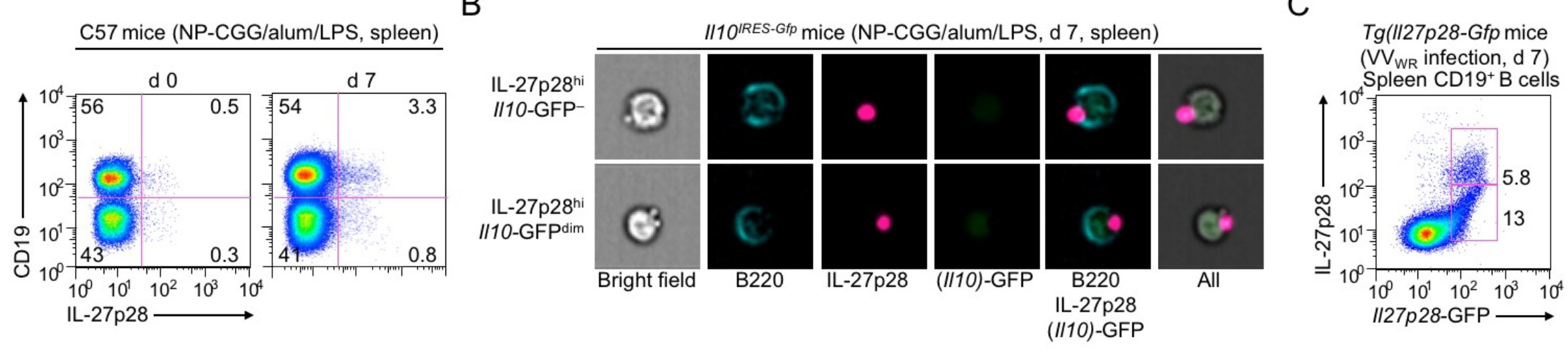

$E$

D

$p=\begin{array}{llllll}0.037 & 0.022 & 0.001 & 0.001 & 0.17 & 0.12\end{array}$

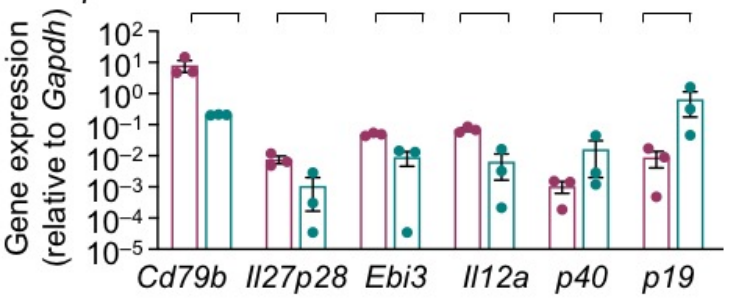

- 57 + NP-CGG/

- $\mu \mathrm{MT}$ alum/LPS (d 14)

G

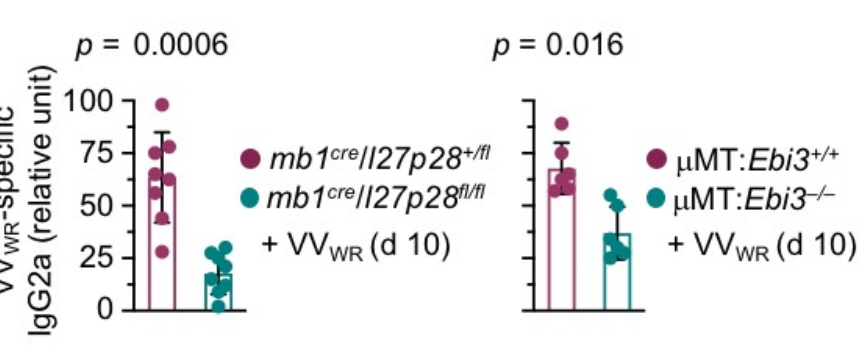

$\mathrm{F}$

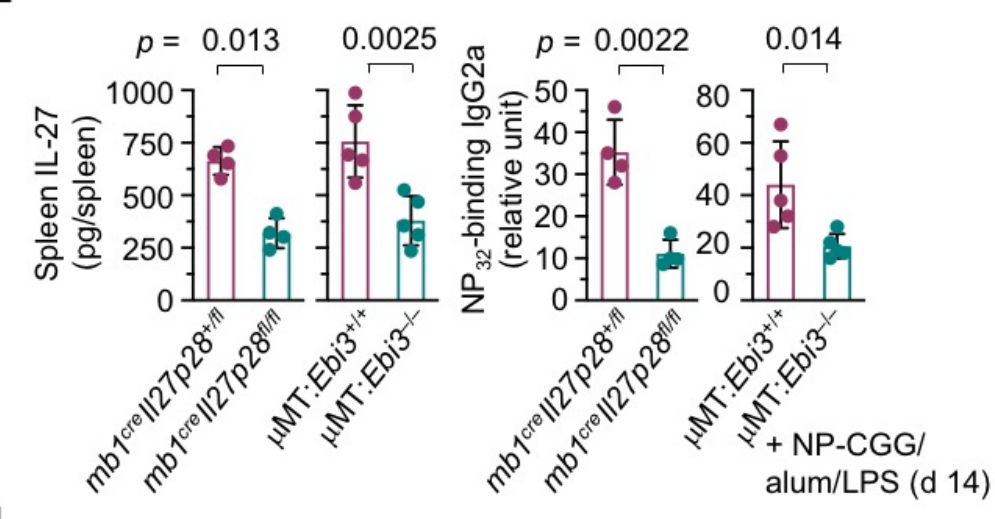

$\mathrm{H}$

$$
p<0.0001 \quad p=0.021 \quad p=0.045 \quad p=0.169
$$

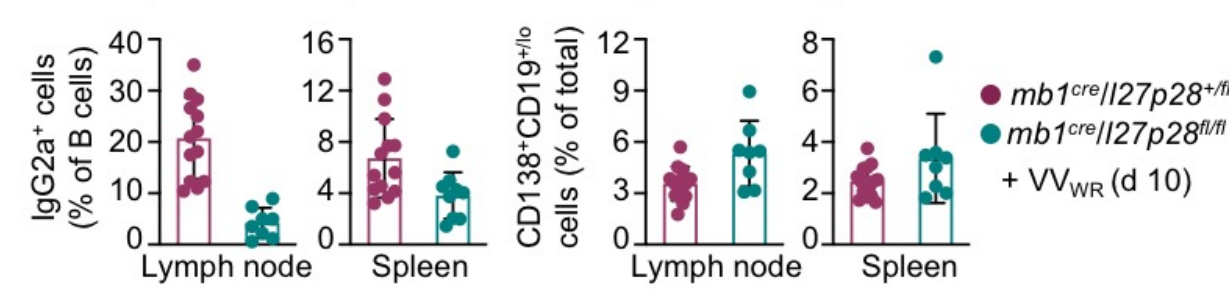

$\operatorname{Tg}$ (II27p28-Gfp mice

$+V_{\text {WR }}$ infection

Mediastinal
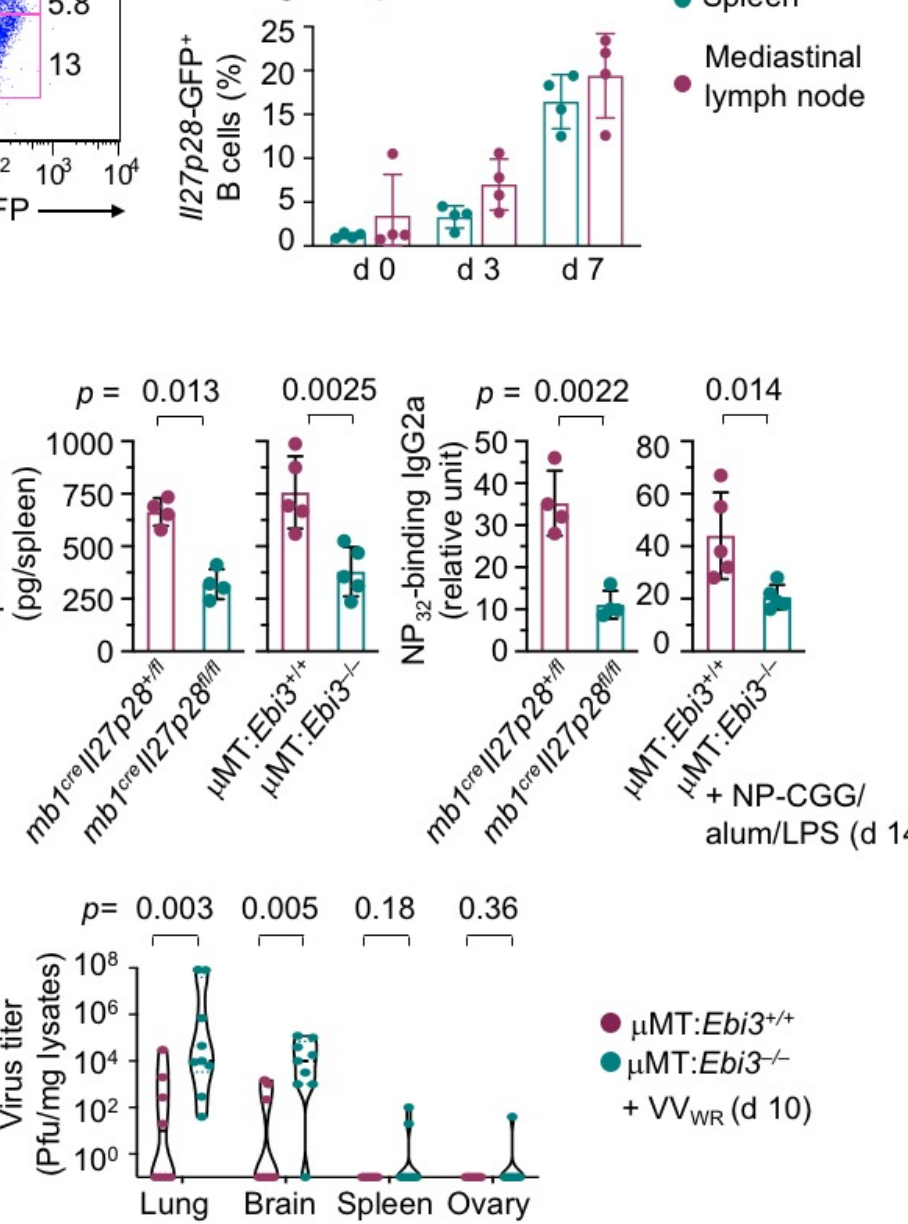


\section{A}

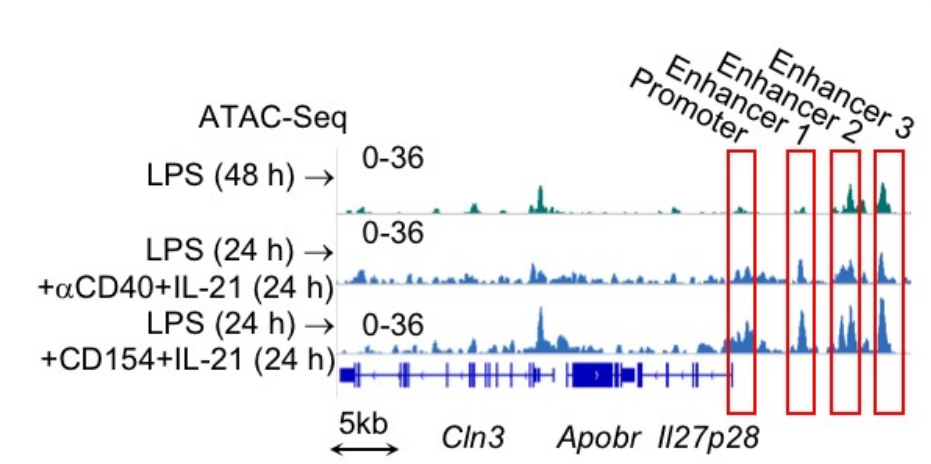

D

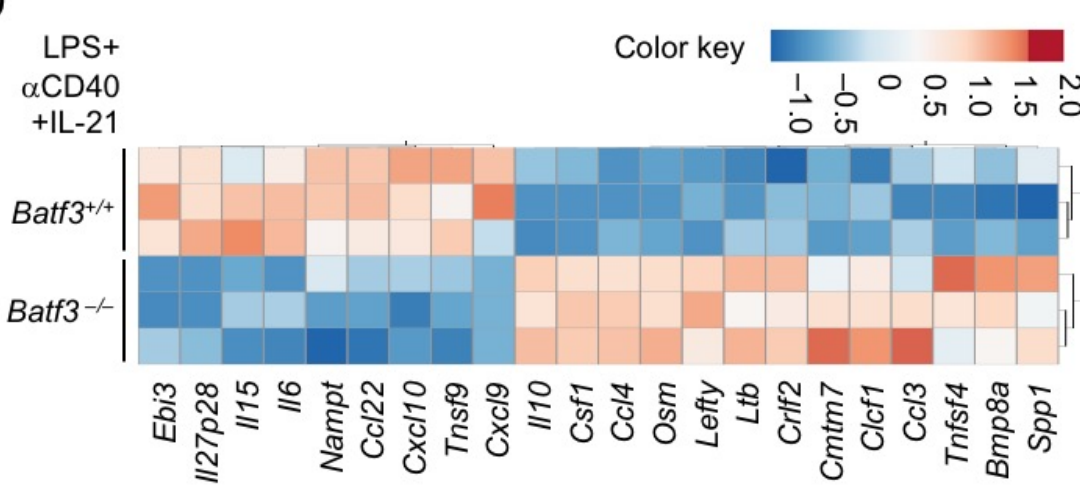

G

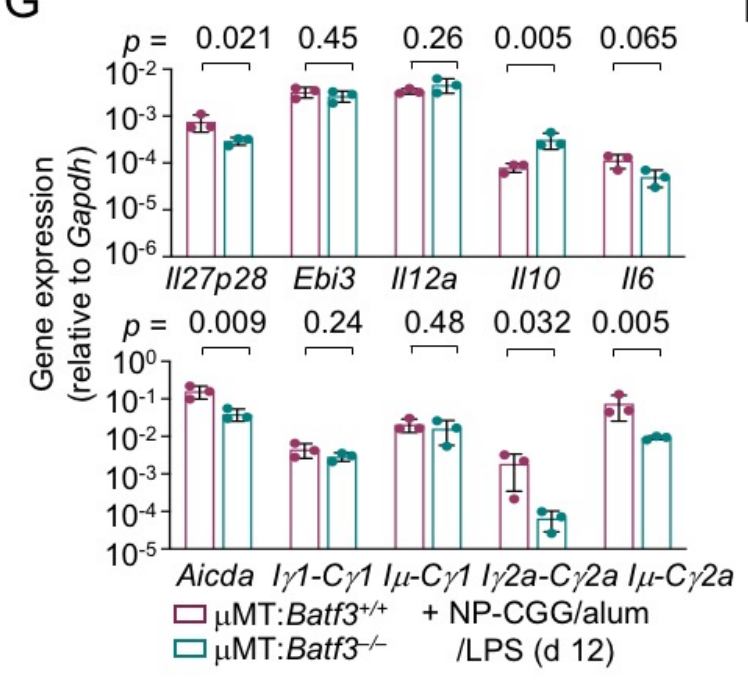

H I

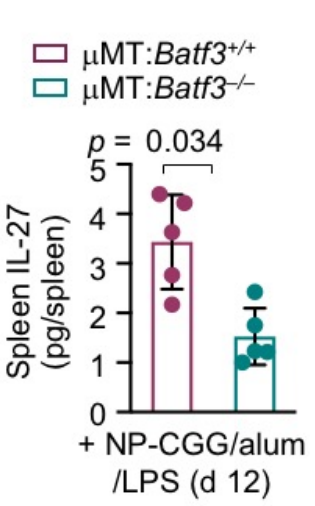

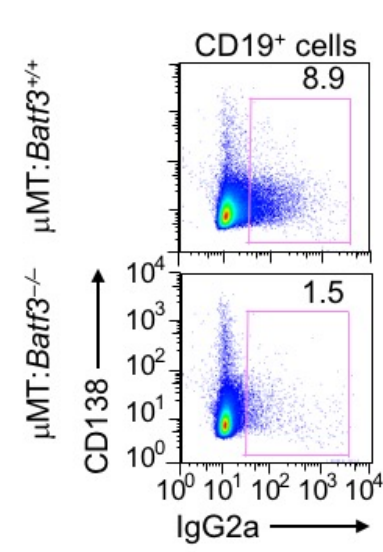

E
C LPS+CD154 LPS

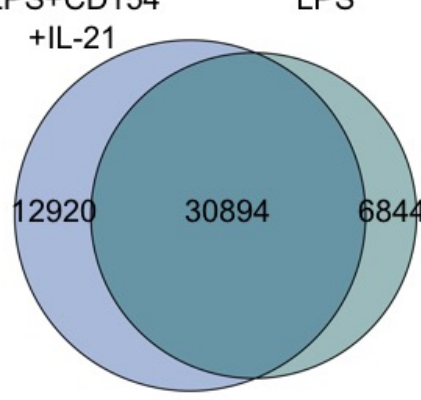

DARs (ATAC-Seq)

DARs in $\mathrm{IL}-27^{+} \mathrm{B}$ cells

Rank (12920 peaks)

1 CCCCCCTAGIGG 1e-764 CTCFL

2 ACTTCCICC 1 e-510 Sfpi1

3 ATGACTCATC 1e-471 Fra1

4 GGAAATCCCC 1e-391 REL

CTCTCC - 1e-207 PolII 7. 7 1  9 ZCCCCCAGGGGA솟 1e-77 E2A

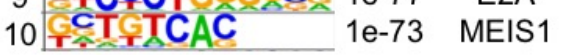

$\mathrm{F}$

$\square$ Batf3 $3^{+/+} \quad$ IL-27 (ng/10 cells)

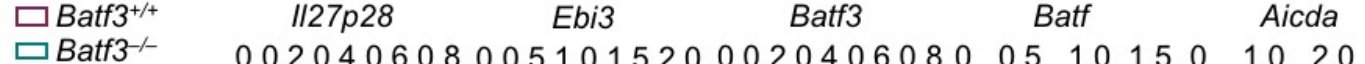

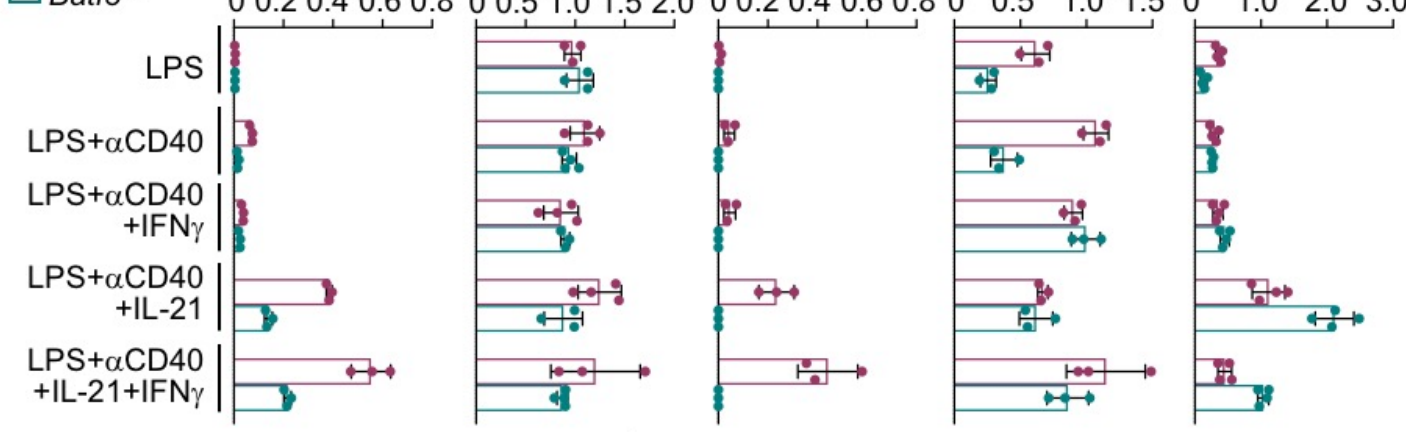

$\square$ Batf3

$\begin{array}{lllllll}0 & 0.3 & 0.6 & 0.9 & 1.2 & 1.5\end{array}$

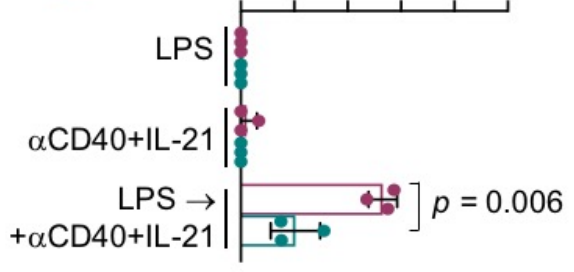

$\mathrm{J}$

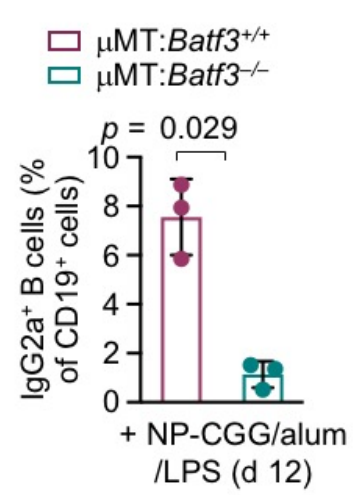

Correlation coefficient: 0.799 $p$ value: $3.3 \times 10^{-10}$

Correlation coefficient: 0.237
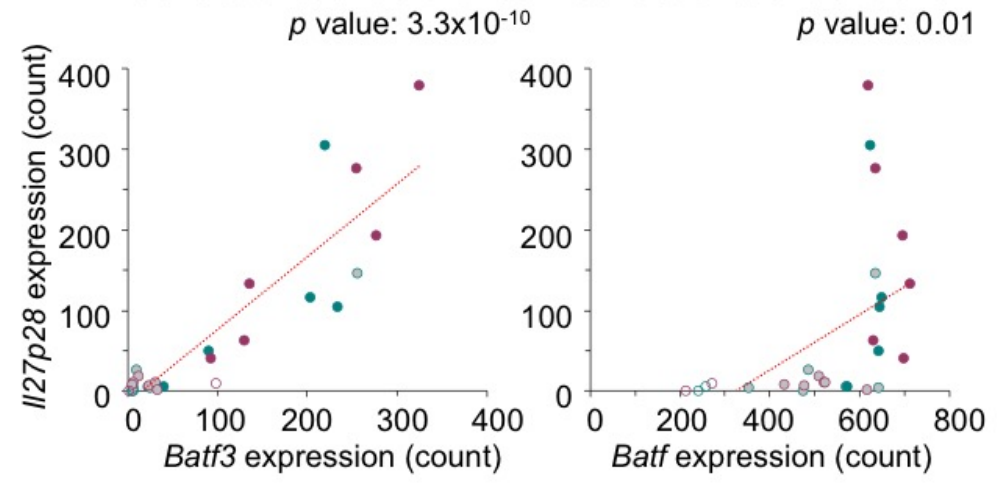

Stimulation: OO None: $\mathrm{d} 0$. OOCSR induction: LPS; CD154; IL-27 induction: LPS+CD154; CD154+IL-21; LPS+CD154+IL-21. o०@Sharpin ${ }^{+/+}$ ○०९Sharpin ${ }^{\text {cpdm/cpdm }}$ 
A

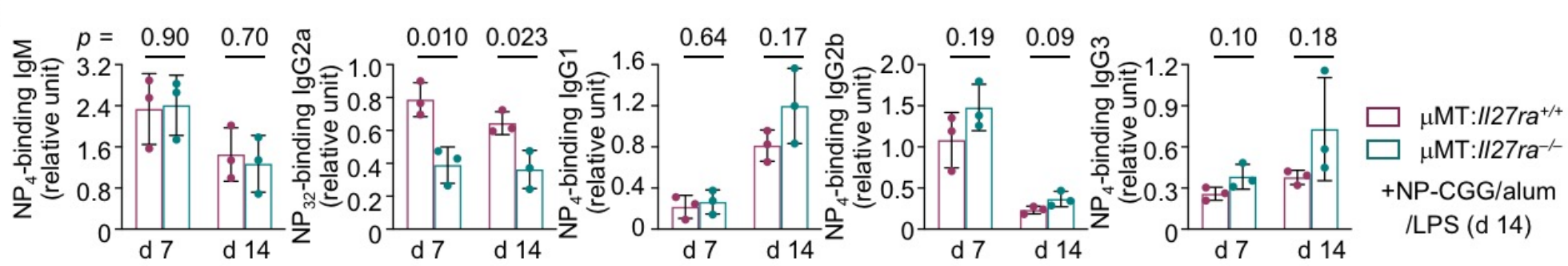

D

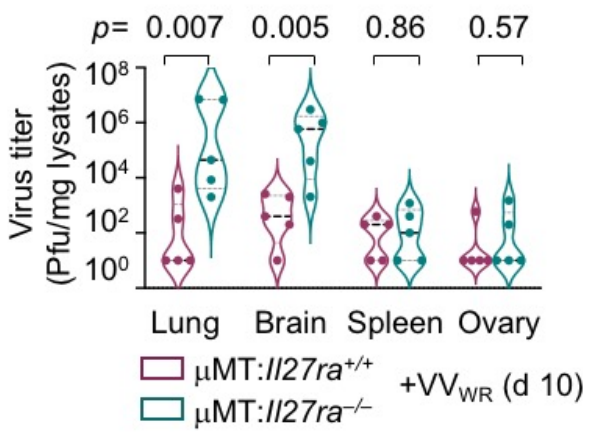

E

G

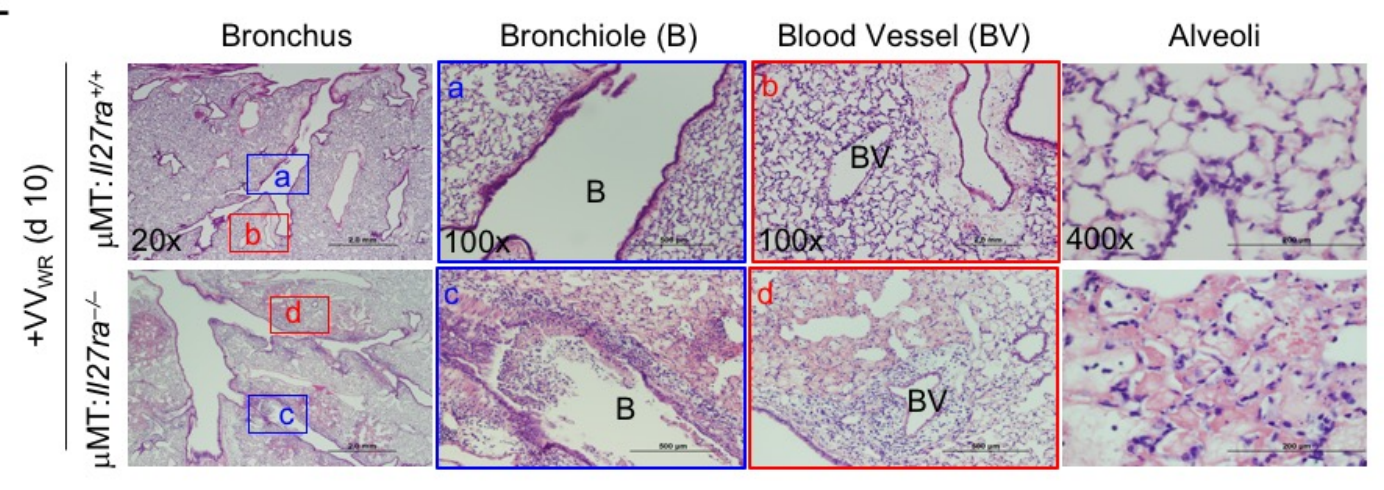

$\frac{1.7 \times 10^{-7}}{4.6 \times 10^{-6}}$

$8.7 \times 10^{-5}$

$\frac{0.0004}{0.83}$

0.83

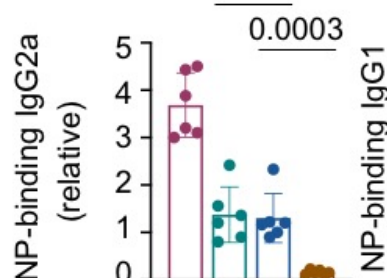

$\square$ MMT:wildtype

$\square^{\mu \mathrm{MT}}:$ :III $7 \mathrm{ra}^{-1}$

$\square \mu \mathrm{MT}: / / 27 \mathrm{ra}^{-\mid}$-lfngri-1-

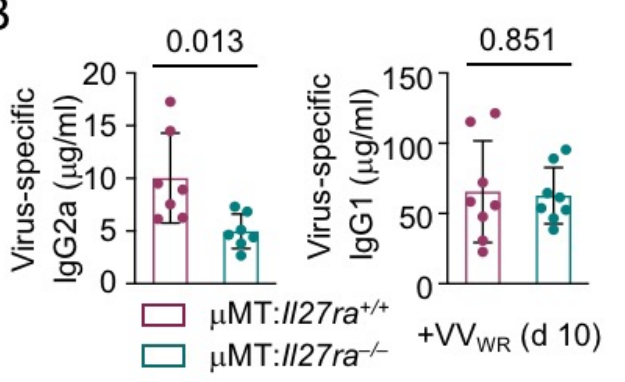

C
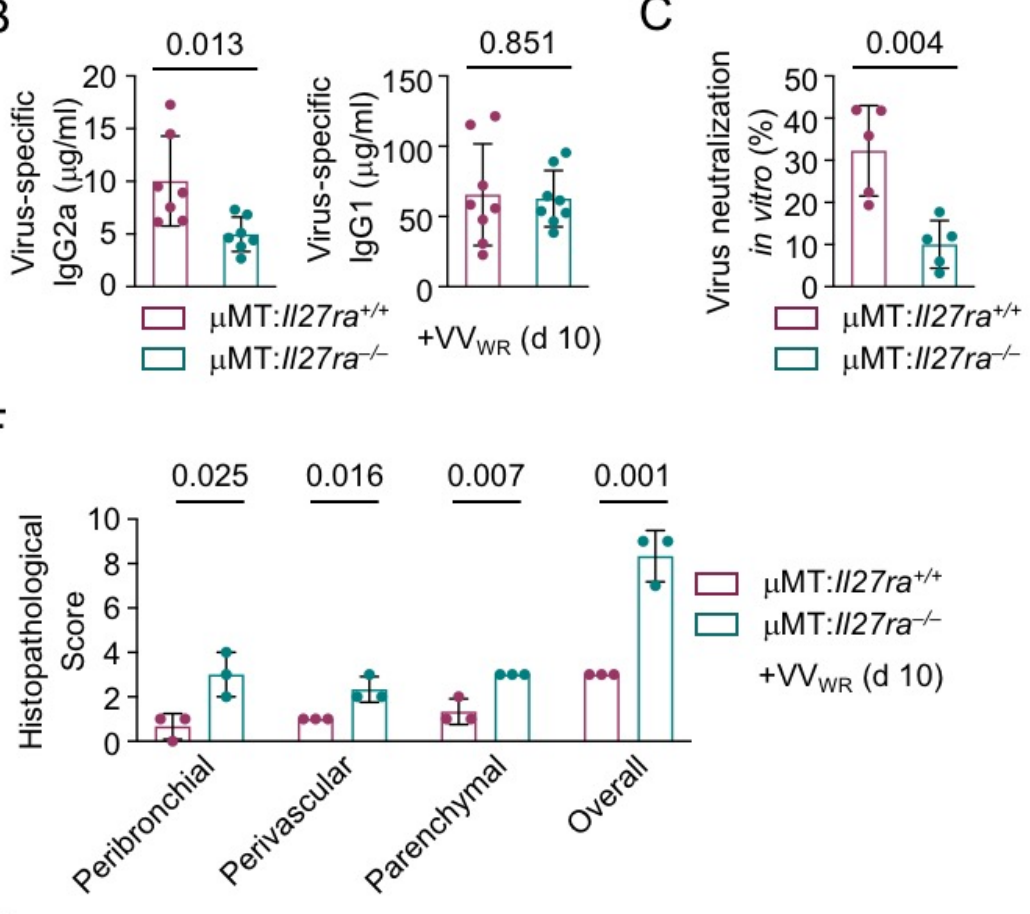

$\mathrm{H}$
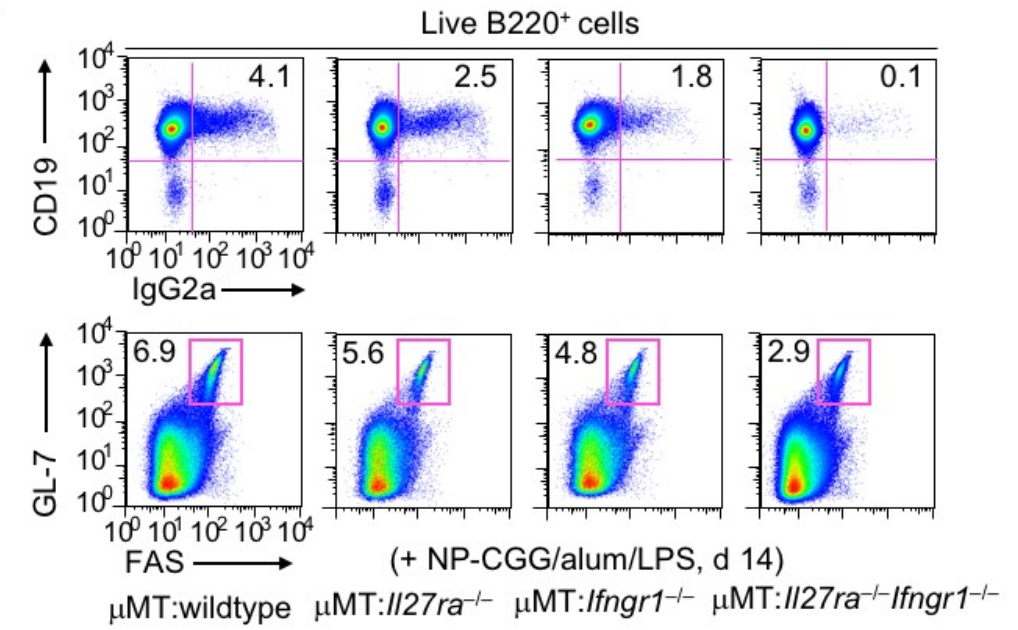
Upregulated genes

B

B

C

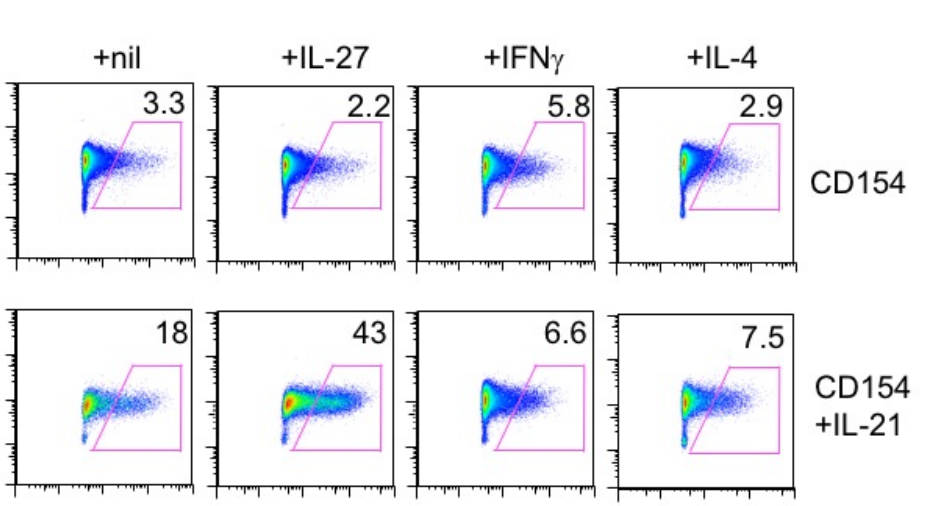

D CD154+IL-27 CD154+IFN vs CD154 vs CD154 vs CD154 vs LPS
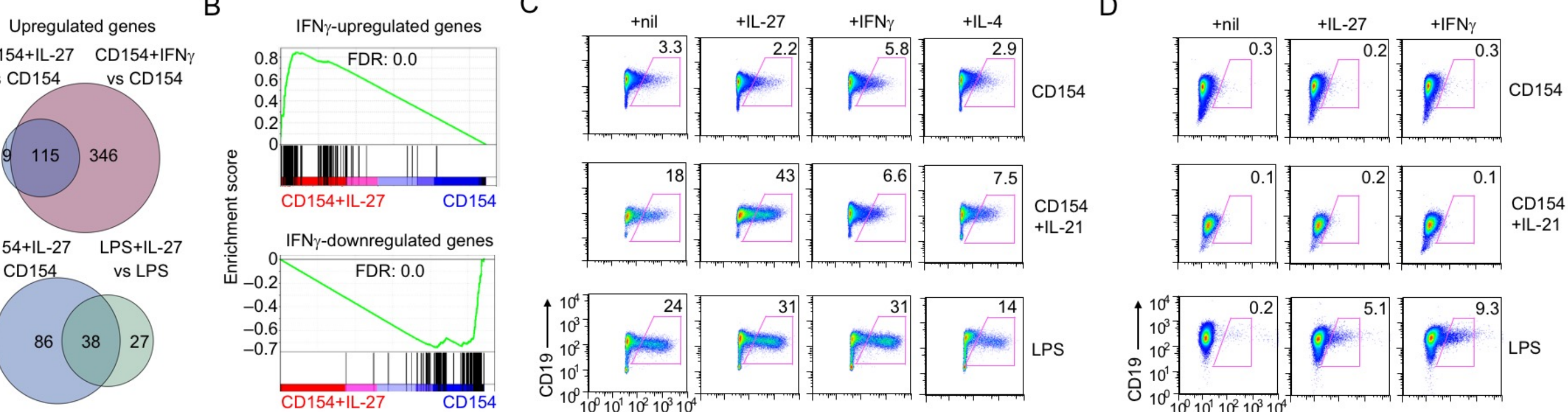

$\square$ LPS
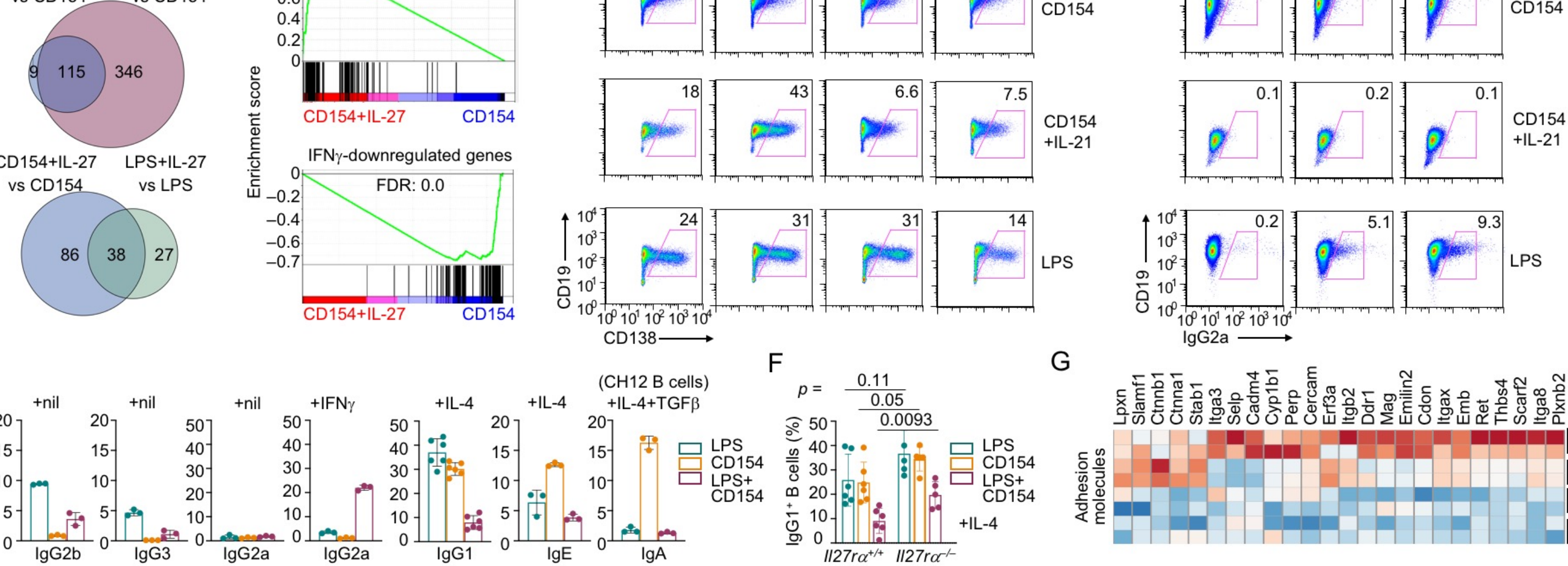

$p=0.0 .0001$

E

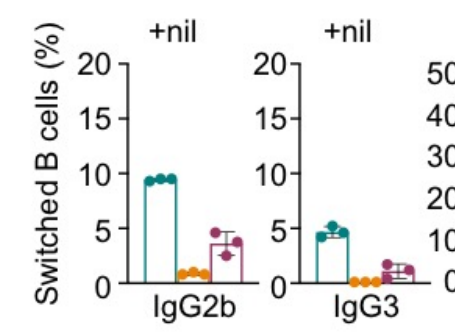

+ nil $\quad+$ IFN $\gamma$

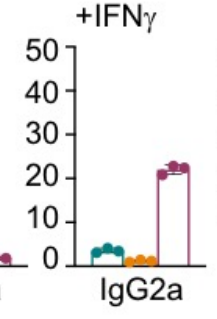

$F$

$p=\frac{0.11}{0.05}$
G

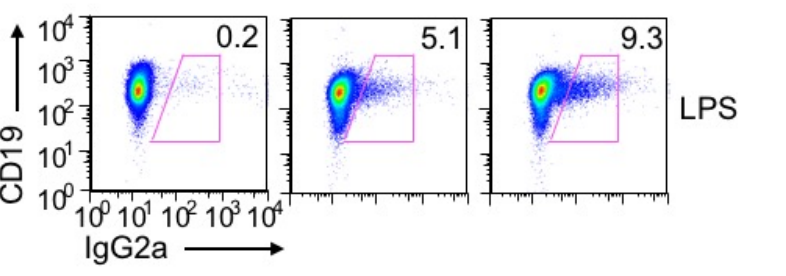

$\infty 20$

我

్ㅡㅇㅛ.

$\begin{array}{ll}0 & 5 \\ 0\end{array}$

0.00004 $20 \underline{0.002}$

○ 15 .

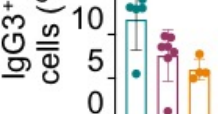

๑50 $\underline{0.0093}$

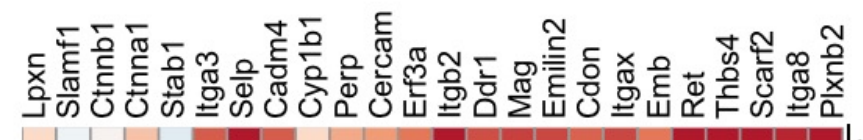

$\square$ LPS

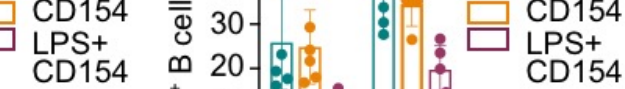

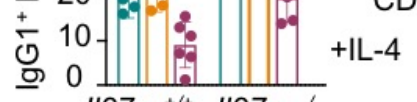

LPS+CD154+IL-2

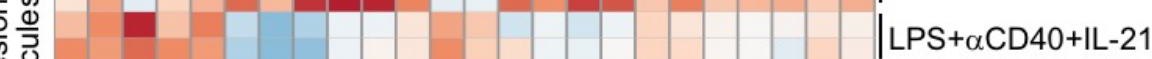
|CD154+IL-4

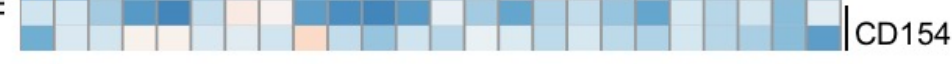
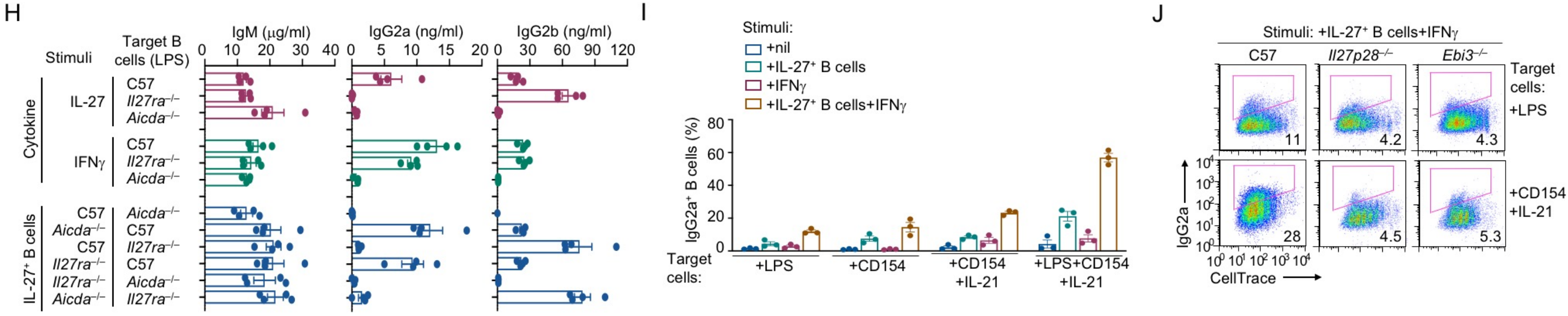


\section{BATF3-dependent induction of IL-27 in B cells bridges the innate and adaptive stages of the antibody response}

Short title: B cells produce IL-27 to mediate the antibody response

One-sentence summary: B cells integrate innate TLR and adaptive CD40 signals to induce BATF3

5 transcription factor for production of IL-27, which together with INF $\gamma$ optimizes antibody responses.

Authors: Hui Yan ${ }^{1}$, Rui Wang ${ }^{1,2,3}$, Jingwei Wang ${ }^{1,2,4}$, Shuai $\mathrm{Wu}^{1,3}$, Maria Fernandez ${ }^{1}$, Carlos E. Rivera ${ }^{1}$, Christian Cervantes ${ }^{1}$, Justin B. Moroney ${ }^{1}$, Xiao-Dong $\mathrm{Li}^{1}$, Nu Zhang ${ }^{1}$, Hong Zan ${ }^{1}$, Xiangzhi Meng ${ }^{1}$, Fushun Zhang ${ }^{1}$, Siyuan Zheng ${ }^{5,6}$, Yidong Chen ${ }^{5,6}$, Zhinan Yin $^{7}$, Ross Kedl ${ }^{8}$, Booki Min ${ }^{9}$, Christopher A. Hunter $^{10}$, Yan Xiang ${ }^{1}$, Paolo Casali ${ }^{1}$ and Zhenming $\mathrm{Xu}^{1, *}$

\section{Supplementary Materials:}

Materials and Methods

Figures S1-S26

Tables S1-S21

Supplementary References and Notes (47-49) 


\section{Materials and Methods}

Mouse strains. C57BL/6J (C57, JAX, stock \#000664), Ebi3 ${ }^{-/-}(\# 008691)$, Batf3 $^{-/-}(\# 013755), \mathrm{Il2}_{\mathrm{ra}}^{-/-}$ (\#018078), Ifng ${ }^{-/-}(\# 002287)$, Ifngrl $^{-/-}(\# 003288)$, Sharpin $^{\text {cpdm/cpdm }}$ (\#007599), Illo ${ }^{\text {IRES-Gfp }}$ (\#008379), $\mu \mathrm{MT}(\# 002288)$ and CD45.1 ${ }^{+}$(Ptprc $\left.{ }^{a}, \# 002014\right)$ were originally from the Jackson Laboratory. Il27p2 $8^{f l f l}$, $20 T g(I l 27 p 28-G f p)$ and $I l 27 r a^{f l f l}$ mice were as described (47-49). Il27p2 fllfl mice were bred with $m b 1^{+/ c r e}$ mice (but not $C d 19^{+/ \text {cre }}$ mice, as the $I l 27 p 28$ and $C d 19$ genes are $<200 \mathrm{~Kb}$ apart on the Chromosome 7) to generate B cell-specific $\mathrm{KO} m b 1^{+/ c r e} I l 27 p 28^{f l f l}$ mice, and $I l 27 \mathrm{ra}{ }^{f l f l}$ mice with $C d 19^{+/ c r e}$ mice to generate

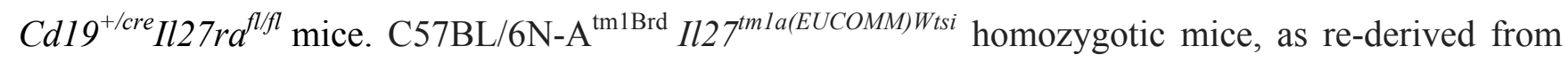
frozen sperms (EMMA Mouse Repository, strain \# 10189), were confirmed to be unable to express

$25 \quad$ Il27p28 transcripts and secrete IL-27 by qRT-PCR and ELISA, respectively, due to the interference of gene expression by the large LacZ-Neo ${ }^{r}$ selection cassette inserted between Exon 1 and 2 (fig. S9A). Hence, they were used as $1127 p 28^{-/}$mice. They were also bred with a C57 mouse strain that expressed Flipase under the control of the Actb locus (JAX, stock \#003800) to delete the cassette, thereby yielding a mouse line carrying the $I l 27^{t m l c}$ allele, as equivalent to the $I l 27 p 28^{f l}$ allele used in this study (fig. S9A).

To generate mice with B cell-specific deficiency in Il27p28, Ebi3, Batf3 or Il27ra using the mixed bone marrow chimera mouse approach, age- and sex-matched recipient C57 mice from the same breeding batch in JAX were treated with neomycin sulfate $(2 \mathrm{mg} / \mathrm{ml}$ in drinking water) for one week before being $\gamma$-irradiated with a lethal dose (1000 rad, or $10 \mathrm{~Gy}, 12.5 \mathrm{~min})$ via a cesium source. After $24 \mathrm{~h}$, mice were randomized into two groups and injected through the tail vein $2.5 \times 10^{6}$ mixed bone marrow cells, as 35 comprised of $80 \%\left(2 \times 10^{6}\right)$ cells from a single $\mu \mathrm{MT}$ mouse and $20 \%\left(0.5 \times 10^{6}\right)$ cells from either a $\mathrm{KO}$ mouse or its age- and sex-matched C57 mouse counterpart ("wildtype"). Prior to mixing, bone marrow cells isolated form tibia and fibula of donor mice were depleted of T cells by incubation with biotinylated anti-CD3 mAb and Magnisort ${ }^{\circledR}$ Streptavidin Negative Selection Beads (eBioscience). Chimeric mice 
were monitored by flow cytometry analysis of CD45, CD19, CD4, CD8, CD11b, CD11c and/or Gr1 expression of mononuclear cells in the circulation for at least 6 weeks to confirm the immune system reconstitution. To verify B cell-specific gene deficiency in mice, $\mathrm{CD} 19^{+} \mathrm{CD} 4^{-} \mathrm{CD} 8^{-}, \mathrm{CD} 19^{-} \mathrm{CD} 4^{+} \mathrm{CD} 8^{-}$ and $\mathrm{CD}^{-} \mathrm{CD}^{+} \mathrm{CD}^{-}$peripheral blood cells were sorted (BD FACSAria ${ }^{\mathrm{TM}}$ II) for genomic DNA extraction and genotyping using PCR involving specific primers. All mice were maintained in a pathogen-free vivarium of the University of Texas Health Science Center at San Antonio (UTHSCSA) 45 and were used for experiments at 8-12 weeks of age, without any apparent infection or disease. While female mice were used in most experiments, in the experiments in which male mice were also used, they did not display significant differences from female mice in the same group.

Mouse immunization and vaccinia virus infection. For immunization, mice were injected intraperitoneally (i.p.) with $100 \mu \mathrm{g}$ of NP-CGG (in average 16 molecules of NP, 4-hydroxy-3-nitrophenyl 50 acetyl, conjugated to one molecule of CGG, chicken $\gamma$-globulin; Biosearch Technologies) in the presence of $100 \mu$ of alum (Imject ${ }^{\circledR}$ Alum adjuvant, ThermoFisher) in the central-left abdomen area and $10 \mu \mathrm{g}$ of LPS (from E. coli, serotype 055:B5 and deproteinized; Sigma-Aldrich) in $100 \mu$ of PBS in the centralright abdomen area. For intranasal route (i.n.) infection of the wildtype Western Reserve (VV $\mathrm{VR}_{\mathrm{WR}}$ ) strain of vaccinia virus (VV), $5 \times 10^{4}$ plaque forming units (Pfu) of infectious viral particles (in $20 \mu 1$ PBS) were

55 delivered into the nostrils $(10 \mu \mathrm{l}$ each) in infect mice, as anesthetized by ketamine and xylazine (50 $\mathrm{mg} / \mathrm{Kg}$ and $5 \mathrm{mg} / \mathrm{Kg}$ of body weight, respectively). Infected mice were kept on a warm pad until waking up and being transferred to original cages. Each mouse was weighed one day before infection and daily after infection until moribund (e.g., losing $25 \%$ or more of the initial body weight) or sacrificing at predetermined end-points. All protocols were in accordance with the rules and regulations of the 60 Institutional Animal Care and Use Committee (IACUC) of UTHSCSA.

Vaccinia virus. HeLa cells $\left(\mathrm{ATCC}^{\circledR} \mathrm{CCL} 2.2^{\mathrm{TM}}\right.$ ) were maintained in monolayer in $150 \mathrm{~cm}^{2}$ tissue culture 
flask in DMEM supplemented with fetal bovine serum (FBS, 10\% v/v, Invitrogen) and penicillinstreptomycin/amphotericin B (1\% v/v). At the time of infection, $10^{9}$ cells growing at the log phase were infected with $\mathrm{VV}_{\mathrm{WR}}$ virus according to a standard protocol at an m.o.i. of 1 for $72 \mathrm{~h}$ at $37^{\circ} \mathrm{C}$ in the presence of DMEM and FBS. Infected cells were pelleted and resuspended in $14 \mathrm{ml}$ of buffer (10 mM Tris-Cl, $\mathrm{pH}$ 9.0). After homogenization in a glass Dounce homogenizer, nuclei were removed by centrifugation $\left(900 \mathrm{~g}, 5 \mathrm{~m}, 5^{\circ} \mathrm{C}\right)$. The supernatant was sonicated and layered onto a cushion of $17 \mathrm{ml}$ of $36 \%$ sucrose. After centrifugation $\left(32,900 \mathrm{~g}, 80 \mathrm{~min}, 4^{\circ} \mathrm{C}\right)$, the virus pellet was resuspended in $1 \mathrm{ml}$ of 1 $\mathrm{mM}$ Tris- $\mathrm{Cl}(\mathrm{pH} 9.0)$. After titration, viruses were stored in $100 \mu 1$ aliquots at $-80^{\circ} \mathrm{C}$ until thawed to be used only once for infection.

For titration of virus titers in tissues, a portion of the lung, spleen as well as brain and ovary (two target organs) isolated from infected mice were weighed and homogenized in $1 \mathrm{ml}$ PBS. The homogenates were 10-fold serially diluted and added to Vero cells (ATCC ${ }^{\circledR}$ CCL-81 ${ }^{\mathrm{TM}}$ ) grown at the log-phase in 6-well plates in the presence of DMEM supplemented with $1 \%$ FBS and $0.5 \%(\mathrm{w} / \mathrm{v})$ 75 methylcellulose (Sigma-Aldrich). After $2 \mathrm{~d}$ of incubation at $37^{\circ} \mathrm{C}$, the medium was removed and cells were washed once before addition of crystal violet $(0.1 \%)$ for incubation at RT for $1 \mathrm{~h}$. After drying, the number of plaques was counted for quantification.

For virus neutralization assay, purified $\mathrm{VV}_{\mathrm{WR}}$ was mixed with serum samples from virus-infected mice at $4^{\circ} \mathrm{C}$ for $1 \mathrm{~h}$ (in a total volume of $1 \mathrm{ml}$ ). For each neutralization condition, three independent mixtures were set up and inoculated onto monolayers of Vero cells in 6-well plate. After incubation at $37^{\circ} \mathrm{C}$ for $1 \mathrm{~h}$, the inoculum was replaced with $2 \mathrm{ml}$ DMEM supplemented with $1 \% \mathrm{FBS}$ and $0.5 \%(\mathrm{w} / \mathrm{v})$ methylcellulose. The plates were incubated at $37^{\circ} \mathrm{C}$ for $2 \mathrm{~d}$ before being processed for plaque counting in the same way as the virus titration analysis.

Mouse B cell isolation and purification. Mouse leukocytes were isolated from single cell suspensions 
85 prepared from the spleen, mediastinal lymph node, and pooled axillary, inguinal and cervical lymph nodes using a 70- $\mu \mathrm{M}$ cell strainer. Lymph node cells were collected in RPMI 1640 medium (Invitrogen) supplemented with FBS (10\% v/v, Invitrogen), penicillin-streptomycin/amphotericin B (1\% v/v) and 50 $\mu \mathrm{M} \beta$-mercaptoethanol (RPMI-FBS) and resuspended in PBS for purification. Spleen cells were resuspended in ACK Lysis Buffer (Lonzo) to lyse red blood cells and, after quenching with RPMI-FBS, 90 were resuspended in PBS for purification. To prepare leukocytes from mouse peripheral blood, freshly collected blood $(100 \mu \mathrm{l})$ were treated with heparin $(5,000 \mathrm{U})$ to avoid aggregation and then subjected to red blood cell lysis using ACK Lysis Buffer (100 $\mu$ l) twice and suspended with RPMI-FBS.

To purify B cells, spleen and lymph node cells were subjected to negative selection (against CD43, CD4, CD8, CD11b, CD49b, CD90.2, Gr-1 or Ter-119) using the EasySep ${ }^{\mathrm{TM}}$ Mouse B cell Isolation Kit 95 (StemCell ${ }^{\mathrm{TM}}$ Technologies). Cell preparations were stained with fluorophore-conjugated antibodies specific for CD45 or CD45.2, CD19, CD4, CD8, CD11b, CD11c and/or Gr1 at $4^{\circ} \mathrm{C}$ for $30 \mathrm{~m}$ and verified by FACS analysis to contain $>95 \% \mathrm{CD} 19^{+} \mathrm{CD} 11 \mathrm{~b}^{-} \mathrm{Gr} 1^{-} \mathrm{CD} 11 \mathrm{c}^{-} \mathrm{B}$ cells and no $\mathrm{CD} 4^{+}$or $\mathrm{CD}^{+} \mathrm{T}$ cells. For further purification, these B cell preparations were sorted (BD FACSAria IITM) to yield $99 \%$ of $\mathrm{CD}_{19} \mathrm{CD}^{+} 1 \mathrm{~b}^{-} \mathrm{Gr} 1^{-} \mathrm{CD} 11 \mathrm{c}^{-}$cells. To purify $\mathrm{CD} 9^{+} \mathrm{B}$ cells by positive selection, single cell suspensions 100 containing $5 \times 10^{6}$ splenocytes were incubated with $2 \mu \mathrm{g}$ biotinylated anti-CD19 mAb before reacted with Magnisort ${ }^{\circledR}$ Streptavidin Positive Selection Beads (eBiosciences) following manufacturer's instructions. In vitro B cell culture. To culture mouse B cells purified from the spleen or lymph nodes, or total splenocytes, cells were cultured $\left(3 \times 10^{5}\right.$ cell $/ \mathrm{ml}, 2 \mathrm{ml}$ in general in a 24-well plate) in RPMI-FBS with the following stimuli at the dose indicated unless specified otherwise: CD154 (3 U/ml), agonistic anti$105 \mathrm{CD} 40 \mathrm{mAb}$ (clone FGK4.5, $5 \mu \mathrm{g} / \mathrm{ml})$, LPS $(3 \mu \mathrm{g} / \mathrm{ml}), \mathrm{TLR} 1 / 2$ ligand $\mathrm{Pam}_{3} \mathrm{CSK}_{4}(100 \mathrm{ng} / \mathrm{ml}$, Invivogen), TLR4 ligand lipid A (1 $\mu \mathrm{g} / \mathrm{ml}$, Invivogen), TLR7 ligand R-848 (30 ng/ml, Invivogen), TLR9 ligand ODN1826 (sequence 5'-TCCATGACGTTCCTGACGTT-3') with a phosphorothioate backbone (CpG, 
$1 \mu \mathrm{M}$; Eurofins), F(ab')2 of a goat anti-mouse $\mu$ chain antibody (anti- $\mu \mathrm{F}\left(\mathrm{ab}^{\prime}\right) 2,1 \mu \mathrm{g} / \mathrm{ml}$; Southern Biotech), which crosslinks IgM BCR, or anti-Ig $\delta$ mAb (clone 11-26c conjugated to dextran, anti- $\delta /$ dex, $110100 \mathrm{ng} / \mathrm{ml}$; Fina Biosolutions), which crosslinks IgD BCR, alone or in combination. CD154 was prepared as membrane fragments isolated from Sf21 insect cells infected by CD154-expressing baculovirus, as we described $(2,28)$ - membrane fragments from non-infected Sf21 cells failed to activate B cells to proliferate. Other stimuli added to the culture included recombinant IL-21 (2 ng/ml), IL-27 (100 ng/ml, R\&D Systems), IFN $\gamma(30 \mathrm{ng} / \mathrm{ml})$ and IL-4 (4 ng/ml), at the indicated dose unless 115 specified otherwise. In sequential stimulation experiments, B cells were cultured in the presence of the first stimulus (or stimuli) for $24 \mathrm{~h}$ before the second stimulus (or stimuli) were added to the culture.

In B cell co-culture experiments, B cells prepared from CD45. $1^{+}$or $\mathrm{CD} 45.2^{+}$mice were stimulated with LPS, CD154 (or $\alpha$ CD40) and/or IL-21 for 36 h, washed twice with RPMI-FBS to remove the stimuli and resuspended in RPMI-FBS before used as stimulating B cells. Target B cells were labeled with 120 CellTrace ${ }^{\mathrm{TM}}$ Yellow and activated for 16-24 $\mathrm{h}$ by stimuli, as indicated, before mixed with stimulating B cells $\left(\mathrm{CD} 45.2^{+}\right.$or $\left.\mathrm{CD} 45.1^{+}\right)$at the $1: 1$ ratio $\left(3 \times 10^{5}\right.$ cells $/ \mathrm{ml}$ each $)$, alone or with other cytokines, and cocultured for additional $72 \mathrm{~h}$ before FACS analyses. Stimulating and target B cells were also co-cultured at different ratios for different time periods, as indicated, before doublet formation analysis.

Bone marrow derived macrophage differentiation and stimulation. Bone marrow cells $\left(10^{7}\right)$ isolated 125 from tibia and fibula were cultured for $10 \mathrm{~d}$ in RPMI-FBS in the presence of conditioned media from L929 cells that express macrophage colony-stimulating factor (which promotes differentiation into macrophages), resulting in the generation of $\mathrm{CD}_{11} \mathrm{~b}^{+}$cells in over $90 \%$ of all cells. After stimulation with LPS (3 $\mu \mathrm{g} / \mathrm{ml}$ or as indicated), CD154 (3 U/ml or as indicated) and/or IL-21 (2 $\mathrm{ng} / \mathrm{ml})$, for $24 \mathrm{~h}$, supernatants were collected for ELISA of IL-27 and cells for RNA extraction and transcript analysis. 
130 Flow cytometry and intracellular staining. To analyze B cells stimulated in vitro, cells were harvested, stained with fluorochrome-conjugated mAbs in Hank's Buffered Salt Solution plus 0.1\% BSA (BSAHBSS) for $20 \mathrm{~m}$ and analyzed for $\operatorname{Ig} \gamma 3$, Ig $\gamma 1$, Ig $2 \mathrm{~b}$ and $\operatorname{Ig} \gamma 2 \mathrm{a}$ expression (CSR to $\operatorname{IgG} 3, \operatorname{IgG} 1, \operatorname{IgG} 2 \mathrm{~b}$ and IgG2a), CD138 (plasma cell marker) and other B cell surface molecules on a flow cytometer (BD LSR II ${ }^{\mathrm{TM}}$ or Celesta $\left.{ }^{\mathrm{TM}}\right)$. To analyzed immune cells in vivo, spleen or blood cells $\left(2 \times 10^{6}\right)$ were first stained 135 with fluorophore-labeled mAbs to surface markers in the presence of mAb Clone 2.4G2, which blocks Fc $\gamma I I I$ and Fc $\gamma I I$ receptors, and FVD-506. After washing, cells were resuspended in HBSS for FACS.

To analyze expression of IL-27p28 and EBI3, cells (2x10 cells) were incubated with Brefeldin A $(100 \mu \mathrm{M})$ in RPMI-FBS $(2 \mathrm{ml})$ at $37^{\circ} \mathrm{C}$ for $4 \mathrm{~h}$. After pelleting, cells were stained with fluorophorelabeled mAbs to surface markers and FVD. After washing, cells were resuspended in the BD 140 Cytofix/Cytoperm ${ }^{\mathrm{TM}}$ buffer $(250 \mu \mathrm{l})$ and incubated at $4^{\circ} \mathrm{C}$ for $20 \mathrm{~m}$. After washing twice with the BD Perm/Wash ${ }^{\mathrm{TM}}$ buffer, cells were counted again and $10^{6}$ cells were resuspended in $100 \mu \mathrm{l}$ of BD Cytofix/Cytoperm ${ }^{\mathrm{TM}}$ buffer for staining with APC- or PE-Cy7-conjugated anti-IL27p28 mAb, PE-Cy7conjugated anti-EBI3 mAb or isotype-matched control $\mathrm{IgG} \mathrm{Abs}$ at $4^{\circ} \mathrm{C}$ for $30 \mathrm{~m}$. After washing with $\mathrm{BD}$ Perm/Wash ${ }^{\mathrm{TM}}$ buffer, cells were analyzed by FACS. All data were analyzed by FlowJo ${ }^{\circledR}(\mathrm{BD})$.

145 B cell proliferation and survival. For flow cytometry analysis of B cell proliferation in vivo, mice were injected twice i.p. with $2 \mathrm{mg}$ of bromodeoxyuridine (BrdU) in $200 \mu \mathrm{PBS}$, with the first and second injection at $24 \mathrm{~h}$ and $20 \mathrm{~h}$ prior to sacrificing, respectively. Spleen B cells were analyzed for BrdU incorporation (by anti-BrdU mAb staining) and DNA contents (by 7-AAD staining after cells were permeabilized) using the APC BrdU Flow kit ${ }^{\circledR}(\mathrm{BD})$ following the manufacturer's instructions. For B 150 cell proliferation analysis in vitro, B cells were labeled with CellTrace ${ }^{\mathrm{TM}}$ CFSE or CellTrace ${ }^{\mathrm{TM}}$ Yellow (ThermoFisher) following the manufacturer's instructions and then cultured for $96 \mathrm{~h}$. Cells were analyzed by flow cytometry for CellTrace ${ }^{\mathrm{TM}}$ CFSE or CellTrace ${ }^{\mathrm{TM}}$ Yellow intensity, which was reduced 
by half after completion of each cell division, as CellTrace ${ }^{\mathrm{TM}}$ CFSE or CellTrace ${ }^{\mathrm{TM}}$ Yellow-labeled cell constituents were equally distributed between daughter cells. The number of B cell divisions was 155 determined by the Flow $\mathrm{Jo}^{\circledR}$ software to calculate the average cell divisions that B cells had completed. To analyze survival of in vitro stimulated B cells, cells were collected and stained with anti-CD19 Ab and $\mathrm{FVD}$ or 7-AAD to distinguish live cells $\left(\mathrm{FVD}^{-}\right.$or $\left.7-\mathrm{AAD}^{-}\right)$from cells undergoing apoptosis/necrosis. ELISA. To determine titers of total IgM, IgG1, IgG2a, IgG2b and IgG3, sera were first diluted 4- to 128-fold with PBS (pH 7.4) plus 0.05\% (v/v) Tween-20 (PBST), and cell culture supernatants were diluted 2- to 10-fold with PBS. Two-fold serially diluted samples and standards for each Ig isotypes were incubated in 96-well plates pre-treated with sodium carbonate/bicarbonate buffer ( $\mathrm{pH} 9.6)$ and coated with pre-adsorbed goat anti-IgM or anti-IgG (to capture IgG1, IgG2a, IgG2b and IgG3) Abs (all $1 \mathrm{mg} / \mathrm{ml})$. After washing with PBST, captured Igs were detected with biotinylated anti-IgM, $-\operatorname{IgG} 1,-$ IgG2a, -IgG2b or -IgG3 Abs, followed by reaction with horseradish peroxidase (HRP)-labeled 165 streptavidin (Sigma-Aldrich), development with o-phenylenediamine (OPD) and measurement of absorbance at $492 \mathrm{~nm}$. Ig concentrations were determined using Prism ${ }^{\circledR}$ (GraphPad) or Excel.

To analyze titers of antigen-specific antibodies, sera were diluted 100- to 1,000-fold in PBST. Twofold serially diluted samples were incubated in a 96-well plate pre-blocked with BSA and coated with $\mathrm{NP}_{32}$-BSA (in average $32 \mathrm{NP}$ molecules on one BSA molecule, to capture all NP-specific antibodies) or $170 \mathrm{NP}_{4}$-BSA (in average $4 \mathrm{NP}$ molecules on one BSA molecule, to capture high-affinity NP-specific antibodies). Captured Igs were detected with biotinylated Ab to IgM, IgG1, IgG2a, IgG2b or IgG3. Data are relative values based on end-point dilution factors or area under curve (AUC).

ELISA of IL-27 in the spleen or circulation in vivo or secreted into the supernatants of cultured B cells in vitro, total spleen lysates, serum samples or supernatants were analyzed by the Mouse IL-27 175 ELISA Read-Set-Go ${ }^{\circledR}$ Kit (Invitrogen) following the manufacturer's' instructions. Briefly, samples were 
diluted two-fold with the diluent and incubated in 96-well plates pre-coated with an anti-IL-27p28 polyclonal Ab. After washing, samples were reacted with a biotin-conjugated anti-EBI3 polyclonal Ab followed by the color development using TMB substrate. IL-27 was quantified using a standard curve derived from the reading of serially diluted purified recombinant IL-27, with a sensitivity of $16 \mathrm{pg} / \mathrm{ml}$.

180 ELISPOT. For ELISPOT analysis of total $\mathrm{IgM}^{+}$and $\mathrm{IgG1}^{+}$antibody secreting cells (ASCs) as well as NP-binding $\operatorname{IgM}^{+}$and $\operatorname{IgG1}^{+}$ASCs, Multi-Screen ${ }^{\circledR}$ filter plates (Millipore) were activated with 35\% ethanol, washed with PBS and coated with pre-adsorbed goat anti-IgM or anti-IgG, or NP $4-B S A(5$ $\mu \mathrm{g} / \mathrm{ml}$ ) in PBS. Single spleen or bone marrow cell suspensions were cultured at 50,000 cells/ml in RPMIFBS at $37^{\circ} \mathrm{C}$ for $16 \mathrm{~h}$. After supernatants were removed, plates were incubated with biotinylated goat 185 anti-mouse IgM or anti-IgG1 Ab, as indicated, for $2 \mathrm{~h}$ and, after washing, incubated with HRP-conjugated streptavidin. Plates were developed using the Vectastain AEC peroxidase substrate kit (Vector Laboratories). The stained area in each well was quantified using the CTL Immunospot software (Cellular Technology) and depicted as the percentage of total area of each well for ASC quantification.

Flow imaging and immunofluorescence microscopy. Splenocytes from immunized $I l 10^{I R E S-G f p}$ mice were first stained for surface B220 and then intracellular IL-27p28 before resuspended in BSA-HBSS at the concentration of $2 \times 10^{7}$ cells $/ \mathrm{ml}$. They were analyzed using an ImageStream X multispectral imaging flow cytometer $\left(\right.$ Amnis $^{\circledR}$ ), with $>10,000$ events collected in each sample. Images were analyzed using IDEAS image-analysis software $\left(\right.$ Amnis $\left.^{\circledR}\right)$, with single-stained samples being used for compensation of fluorescence signals. To visualize IL-27p28 expression and GCs, spleens were embedded in OCT 195 (Tissue-tek) and snap-frozen on dry ice. Cryostat sections $(7 \mu \mathrm{m})$ were fixed in pre-chilled acetone at $-80^{\circ} \mathrm{C}$ for $30 \mathrm{~m}$ and air dried at $25^{\circ} \mathrm{C}$. Sections were stained with fluorochrome-conjugated $\mathrm{Abs}$ at $25^{\circ} \mathrm{C}$ for $1 \mathrm{~h}$ in a moist chamber. After washing with HBSS, sections were mounted using ProLong ${ }^{\circledR}$ Gold Anti-Fade Reagent (Invitrogen) and examined under a Zeiss LM710 confocal microscope. 
200 freshly isolated and purified by positive selection (see above) were pelleted and total RNA was extracted using the RNeasy Mini Kit (Qiagen). For ex vivo analysis of gene expression in the spleen, 1/6 of spleen freshly isolated from each mouse was immediately preserved in the RNAlater ${ }^{\mathrm{TM}}$ stabilization reagent until cDNA synthesis. First-strand cDNA was synthesized from $1 \mu \mathrm{g}$ of RNAs suing the Superscript ${ }^{\mathrm{TM}}$ III system (Invitrogen) and qPCR was performed using SYBR Green (Dynamo HS kit; New England 205 Biolabs) kit in a QuantStudio ${ }^{\text {TM }} 3$ Reak-Time PCR Instrument (Applied Biosystems). The $\Delta \Delta$ Ct method was used to analyze levels of transcripts and data were normalized to the levels of Gapdh or as indicated.

For RNA-Seq, after RNA integrity was verified using an Agilent Bioanalyzer 2100 (Agilent), RNA was processed using an Illumina TruSeq RNA sample prep kit v2 (Illumina). Clusters were generated using TruSeq Single-Read Cluster Gen. Kit v3-cBot-HS on an Illumina cBot Cluster Generation Station. 210 After quality control procedures, individual RNA-Seq libraries were pooled based on their respective 6$\mathrm{bp}$ index portion of the TruSeq adapters and sequenced at $50 \mathrm{bp} /$ sequence using an Illumina HiSeq 3000 sequencer. Resulting reads, typically 20 million reads per sample, were checked by assurance (QA) pipeline and initial genome alignment (Alignment). After sequencing, demultiplexing with CASAVA was employed to generate a Fastq file for each sample. All sequencing reads were aligned with the 215 (GRCm38/mm10) reference genome using HISAT2 default settings, yielding Bam files, which were then processed using HTSeq-count to obtain counts for each gene. RNA expression levels were determined using GENCODE annotation. Differential expression analysis was performed using the Deseq2 package in R post-normalization based on a Benjamini-Hochberg false discovery rate (FDR)-corrected threshold for statistical significance of $p_{a d j}<0.05$ or raw $p$ value $<0.01$. Transcript read counts were transformed 220 to $\ln (\mathrm{x}+1)$ used to generate heatmaps in Clustvis. Volcano plots depicting $\log _{2}$-fold change and raw or adjusted $p$ values were generated in $\mathrm{R}$. 
Assay for Transposase-Accessible Chromatin using Sequencing (ATAC-Seq). For ATAC-Seq library preparation, Omni-ATAC, which minimizes mitochondrial DNA contamination, was used to increase the library complexity and the signal-to-noise ratio. Briefly, stimulated B cells were centrifuged at 500 $225 \mathrm{~g}$ for $5 \mathrm{~min}$ at $4^{\circ} \mathrm{C}$, washed with $50 \mu \mathrm{l}$ cold PBS and centrifuged again at $500 \mathrm{~g}$ for $5 \min$ at $4^{\circ} \mathrm{C}$. The cell pellet was then resuspended in $50 \mu$ cold lysis buffer $(10 \mathrm{mM}$ Tris $\mathrm{pH} 7.4,10 \mathrm{mM} \mathrm{NaCl}, 3 \mathrm{mM}$ $\mathrm{MgCl}_{2}, 0.1 \%$ NP-40, 0.1\% Tween-20, 0.01\% digitonin), incubated on ice for 3 min, quenched with 500 $\mu$ cold Tween-only-buffer (10 mM Tris $\mathrm{pH} 7.4,10 \mathrm{mM} \mathrm{NaCl}, 3 \mathrm{mM} \mathrm{MgCl}, 0.1 \%$ Tween-20). After pelleting by centrifugation $\left(500 \mathrm{~g}, 30 \mathrm{~min}\right.$ at $\left.4^{\circ} \mathrm{C}\right)$, nuclei were immersed in tagmentation reaction mixture $230 \quad\left(5 \mu \mathrm{l} 5 \mathrm{X}\right.$ insertion buffer, $2 \mu \mathrm{l}$ Tn5-50 transposase, $8 \mu \mathrm{l}$ sterile $\mathrm{PBS}, 10 \mu \mathrm{l}$ nuclease-free $\mathrm{H}_{2} \mathrm{O}$ ) at $37^{\circ} \mathrm{C}$ for $45 \mathrm{~min}$, resulting in a $30 \mu \mathrm{l}$ mixture. After dilution in $30 \mu \mathrm{l}$ of $2 \mathrm{X}$ nuclei lysis/deactivation buffer $(300 \mathrm{mM} \mathrm{NaCl}, 100 \mathrm{mM}$ EDTA, $0.6 \% \mathrm{SDS}, 1.6 \mu \mathrm{g}$ Proteinase $\mathrm{K})$ and incubation for $30 \mathrm{~min}$ at $40^{\circ} \mathrm{C}$, DNA was purified using Qiaquick columns (Qiagen) and amplified using Illumina-compatible index primers to prepare the libraries, which were normalized through qPCR analysis. DNA was enriched for 235 fragments $<1000$ bp using Zymo Select-a-Size DNA Clean and Concentrator MagBead kit according to manufacturer's instructions. After the quality check on an Agilent Bioanalyzer 2100, samples were pooled at an equimolar ratio for 50-bp single-end run on a HiSeq 3000. For sequence analysis, ATACSeq data sets were aligned to the mouse genome (GRCm38/mm10) by Bowtie2 with the default parameters. Uniquely mapped reads were masked using Samtools. Peaks were identified by MACS2 240 with FDR (q-value 0.05) comparing specific ATAC reads with unspecific control reads derived from sequencing matching input samples without building the shifting model and set extension size $200 \mathrm{bp}$. Peaks were annotated by annotatePeaks.pl (-d option) in HOMER using the peak file. The unique peaks of each sample were identified by MAnorm 1.3.0. For motif enrichment analysis, unique peaks was selected for extraction of $150 \mathrm{bp}$ centered on the summit, and subjected to motif analysis using HOMER. 
245 Chromatin immunoprecipitation (ChIP) and ChIP-Seq. B cells $\left(1 \times 10^{7}\right)$ were treated with disuccinimidyl glutarate $(1 \mathrm{mM})$ for $30 \mathrm{~min}$ and then washing with cold PBS before chromatin crosslinking by $1 \%$ formaldehyde in $\mathrm{PBS}$ at $25^{\circ} \mathrm{C}$ for $10 \mathrm{~min}$. After quenching with $100 \mathrm{mM}$ of glycine ( $\mathrm{pH}$ 8.0) and washing with cold PBS containing a cocktail of protease inhibitors (Sigma-Aldrich), B cells were resuspended in SDS-lysis buffer $(20 \mathrm{mM}$ Tris-HCl, pH 8.0, $200 \mathrm{mM} \mathrm{NaCl}, 2 \mathrm{mM}$ EDTA, 0.1\% $250(\mathrm{w} / \mathrm{v})$ sodium deoxycholate, $0.1 \%(\mathrm{w} / \mathrm{v})$ SDS, and protease inhibitor cocktail). Chromatin was sonicated to yield approximately 200-600-bp DNA fragments, pre-cleared with agarose beads conjugated with protein A (ThermoFisher) and then incubated with polyclonal rabbit Ab to BATF3 or NF-кB p65. After overnight incubation, immune complexes were isolated using agarose-beads conjugated with protein A, washed and eluted in buffer containing $50 \mathrm{mM}$ Tris-HCl, 0.5\% SDS, $200 \mathrm{mM} \mathrm{NaCl}$ and $100 \mu \mathrm{g} / \mathrm{ml}$ 255 Proteinase $\mathrm{K}(\mathrm{pH} 8.0)$. Eluates were incubated at $65^{\circ} \mathrm{C}$ for $4 \mathrm{~h}$ to reverse formaldehyde crosslinks. DNA was purified using a QIAquick PCR purification column (Qiagen).

For ChIP-Seq, after the size and quality check on an Agilent Bioanalyzer 2100, DNA samples were used to prepare the library for sequencing at a HiSeq 3000. As a control, input DNA was reverse crosslinked and prepared using the same library preparation kit. After mapping of sequencing reads to 260 the mouse mm10 genome and removal of duplicated reads using bowtie2, peak calling was performed by MACS2 using default settings except not building the shifting model and set extension size $200 \mathrm{bp}$. Bigwig files were generated for visualization in the Integrative Genomics Viewer (IGV 2.3.81). Reads in peaks were counted by annotatePeaks.pl (-d option) from HOMER.

Bioinformatics. To investigate biological pathways associated with DEGs in the core transcriptional 265 signature of B cells stimulated in vitro with selected conditions, the ingenuity pathway analysis (IPA, Qiagen) was performed using aggregate mRNA profile was analyzed to identify statistically overrepresented pathways based on FDR-adjusted $\mathrm{p}$ values $<0.05$ and to infer pathway activation status based 
on $\mathrm{Z}$ scores. Alternatively, DEGs were manually curated and compared to multiple public databases, including Gene Ontology (GO) and Kyoto Encyclopedia of Genes and Genomes (KEGG), for enrichment analysis. DEGs were also directly compared to curated gene signatures to identify statistically significant concordance in the expression using the gene set enrichment analysis (GSEA) algorithm. To identify from 1500 curated transcription factors those that were inducible by the same IL-27-inducing stimuli and mediated IL-27 induction, expression of 411 genes with at least 5 counts per million reads in any of the 14 samples of in vitro stimulated B cells and that of $I l 27 p 28$ were subjected to unsupervised pair-wise 275 correlation analysis using the cor function in R, yielding a matrix of correlation coefficients, which were visualized by a heatmap generated using the ggplot2 package in R. For scRNA-Seq, DEGs were subjected to principle component analysis (PCA) to identify subsets.

For ChIP-Seq, analysis of enriched motifs was performed with HOMER to identify bona fide NF- $\kappa \mathrm{B}$ p65 target sites as well as cis-elements that were close to such target sites and occupied by a different transcription factor(s), with which NF- $\mathrm{B}$ interacted with. Known motif enrichment in the peak sets for each stimulus was compared against the whole genome, whereas enrichment in the different differentially bound (DB) groups was compared against all the NF- $\mathrm{kB}$ p65 peaks. Peak location comparison between conditions was done with the mergePeaks command from HOMER using -d 500 setting and -venn option. To identify NF- $\kappa$ B binding to the promoter region of induced B cells, the BETA algorithm was used to integrate the ChIP-Seq and RNA-Seq datasets and return a score of interested genes based on their distance to TSSs (based on ChIP-Seq) and RNA-Seq expression profile.

Immunoblotting. B cells $\left(2 \times 10^{6}\right)$ were resuspended in lysis buffer supplemented with Halt ${ }^{\mathrm{TM}}$ Protease \& Phosphatase Inhibitors Cocktail (ThermoScientific). After sonication, cell lysates were separated by SDS-PAGE and transferred onto PVDF membranes for immunoblotting. Membranes were then stripped with Restore ${ }^{\mathrm{TM}}$ PLUS Western Blot Stripping Buffer (ThermoScientific) for re-blotting. 
Histopathology. $\mathrm{VV}_{\mathrm{WR}}$-infected mice were sacrificed at the pre-determined endpoint by cervical dislocation and infused with $2 \mathrm{ml}$ of $4 \%$ paraformaldehyde through intra-tracheal injection. The fixed lung tissues were harvested and then paraffin embedded for staining of tissue sections $(5 \mu \mathrm{m})$ by hematoxylin and eosin staining. Lung pathology was evaluated by two independent investigators blinded 295 to the identity to examine: 1. alveolar septa widened by swollen mononuclear cells which contain granular eosinophilic cytoplasm; 2. necrosis of cells within alveolar septa, karyorrhexis and mild infiltration of alveolar walls by neutrophils, fibrin; 3. exudation of leukocytes into alveolar air spaces; 4. edema (eosinophilic proteinaceous material within distal air sacs (intra alveolar edema) sometimes intermixed with aggregates of red cells (hemorrhage); 5. aggregates of mononuclear cells 300 (lymphocytes and mononuclear/macrophage) in peribronchiolar and perivascular spaces; 6. the mucosa of bronchioles hyperplastic (pilling up) or extended (type II pneumocyte hyperplasia); desquamated or necrotic epithelial cells occasionally present in the lumens; 7. fibrosis; 8. blood vessel changes, including perivascular and interstitial edema, thrombosis and margination of neutrophils.

Statistical analysis. All statistical analyses were performed using Excel (Microsoft), GraphPad Prism ${ }^{\circledR}$ or R software environment. Differences in RNA transcript expression were determined by Deseq2, which estimate variance-mean dependence in count data and test for differential expression based on a model using the negative binomial distribution. Spearman correlation analysis was used to measure the strength and direction of gene expression correlations. The student $t$-test was used to analyze the group differences, assuming equal variances. The Mantel-Cox test was used for survival analysis.

310 Reagents. All antibodies are listed in Table S19, and the sequence of all primers are listed in Table S20.

\section{Supplementary References and Notes}

47. S. Zhang et al. Hepatology 57, 1620-1631 (2013).

48. J. Do et al. Proc. Natl. Acad. Sci. U.S.A. 114, 10190-10195 (2017).

49. A. M. Kilgore et al. Immunohorizons 2, 1-11 (2018). 


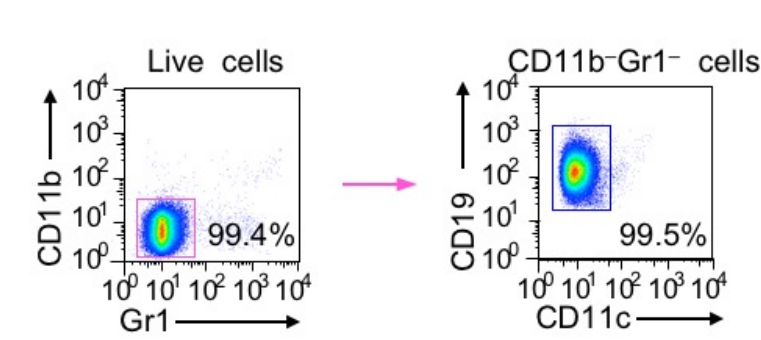

B

$$
\text { Gr1 } \longrightarrow
$$

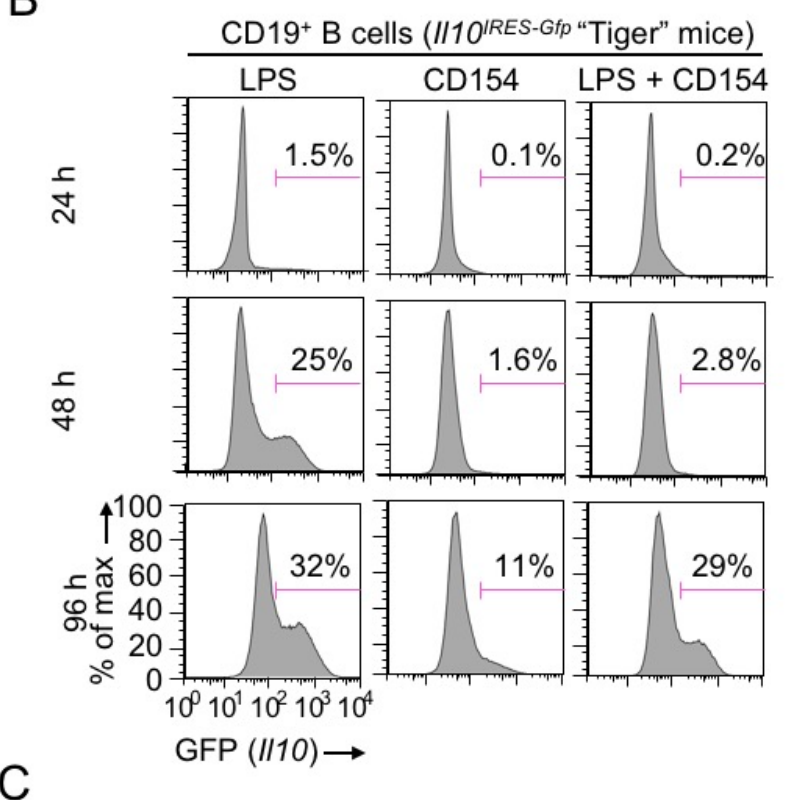

$$
\text { C }
$$

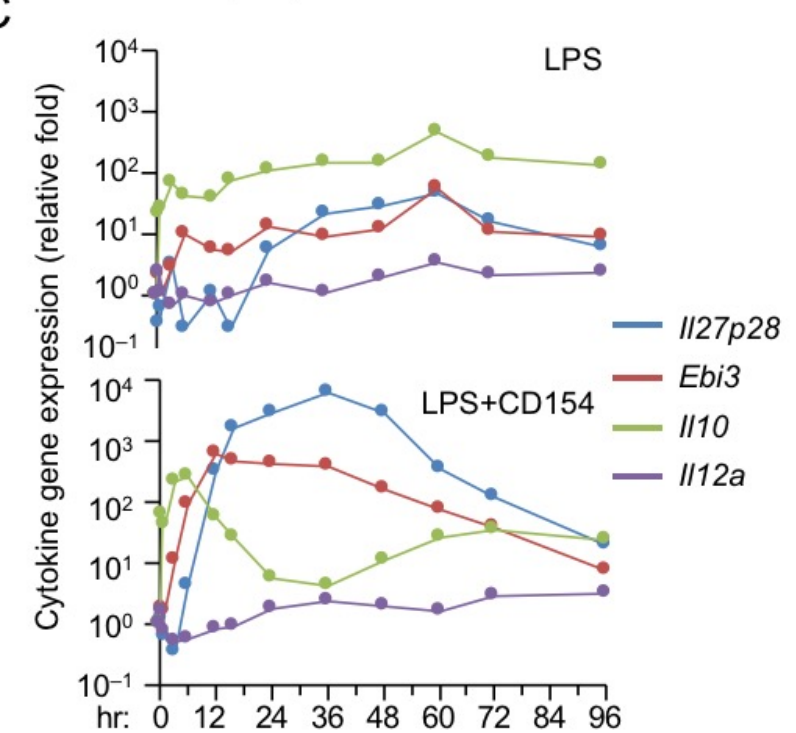

Purified B cells $(24 \mathrm{~h})$
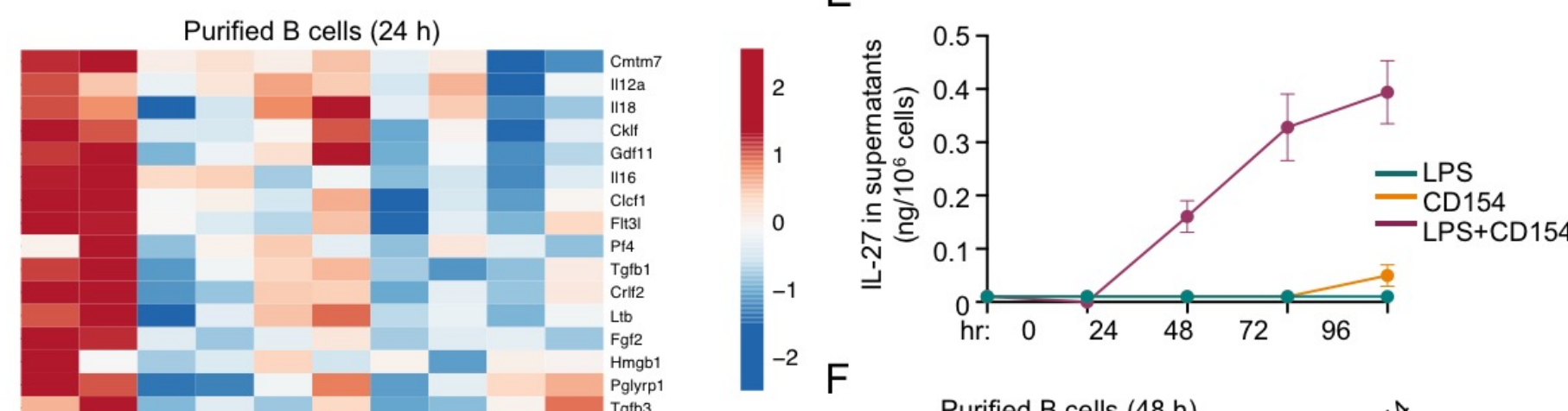

$\mathrm{F}$

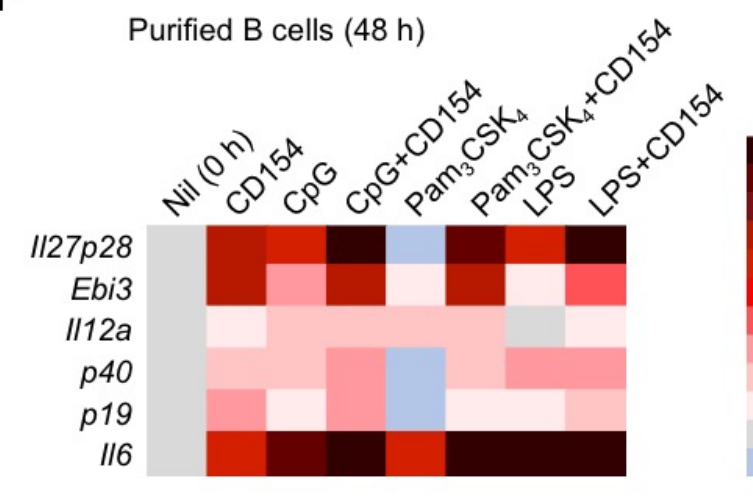

G

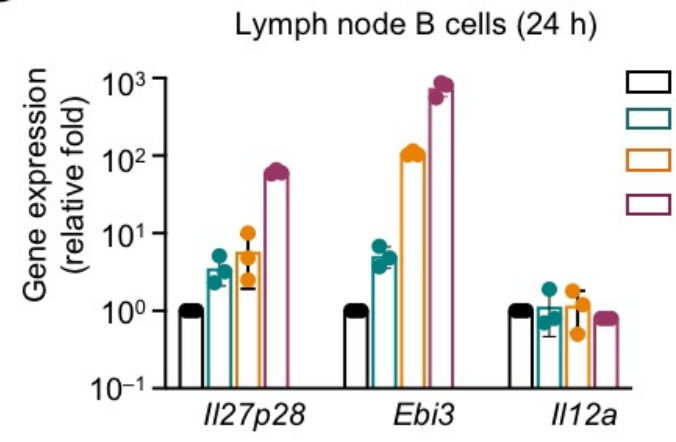

in $B$ cells by TLR ligands plus CD154. (A) Purity of B cells after vitro stimulation. (B) FACS analysis of GFP induction in II10 IRES-Gfp B cells (from II10 1Fly mice, JAX \#008379). (from IIf experiments. (C) qRT-PCR analysis of cytokine gene transcript levels in purified spleen B cells after stimulation at time points as $C d 79 b$ expression and expressed as the ratio to values of freshly isolated B cells $(0 \mathrm{~h})$. Representative of two cells $(0 \mathrm{~h})$. Representative of two independent experiments. (D) Heatmap of expression of genes with
cytokine and chemokine activities (JAXInformatics) differentially expressed in B cells after stimulation, as indicated, for $24 \mathrm{~h}$. Data are from two independent RNASeq experiments. Only genes that were differentially expressed as compared to those in freshly isolated $B$ cells (nil, h 0 ) and showed $>=50$ reads in at least one sample were depicted. (E) ELISA of IL-27 secreted into the supernatants of purified spleen B cells after stimulation, as indicated (mean and s.d., triplicates). (F) qRT-PCR analysis of cytokine transcript levels in purified spleen B cells, as induced by indicated stimuli after $48 \mathrm{~h}$ of stimulation. Data were normalized to stimulation. Data were nossion and expressed as the ratio to values of freshly isolated $B$ cells $(0 \mathrm{~h})$. Representative of two independent experiments. (G) qRTPCR analysis of gene transcript levels in purified lymph node B cells $48 \mathrm{~h}$ after stimulation. Data were normalized to Col79b expression and normalized to Cd7gb expression and freshly isolated B cells. freshly isolated B cells. experiments.

fig. S1 
B

Bone marrowderived macrophages

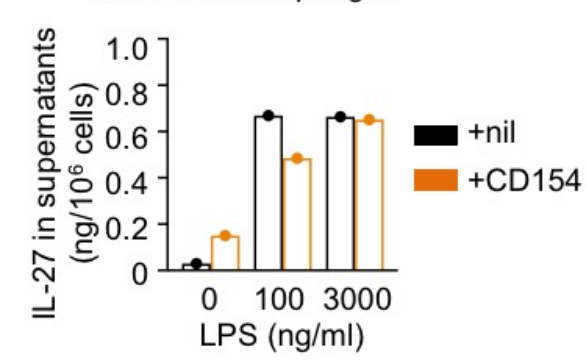

LPS

흥 $10^{0}$

응 $10^{-1}$

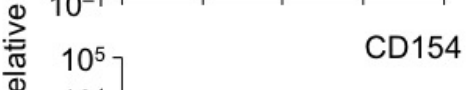

ब $10^{4}$

드 $10^{3}$

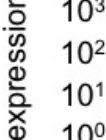

$10^{-1}$

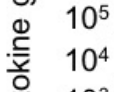

仓े $10^{3}$

$10^{2}$
$10^{1}$
$10^{\circ}$

$10^{0}$
$10^{-1}$

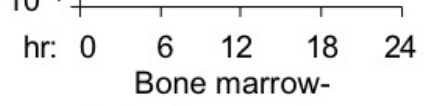

derived macrophages

\section{- $1127 p 28$ \\ - Ebi3 \\ - $1112 a$ \\ - 116}

fig. S2. IL-27 induction in B cells and macrophages. (A) qRT-PCR analysis of gene induction in purified spleen B cells after stimulation, as indicated, for at 24 or $48 \mathrm{~h}$. Data were normalized to $C d 79 \mathrm{~b}$ expression and expressed as the ratio to values of freshly isolated $\mathrm{B}$ cells $(0 \mathrm{~h})$. Representative of two independent experiments. (B) ELISA of IL-27 secreted into the supernatants of bone marrow-derived macrophages after stimulation, as indicated, for 48 h. Representative of two independent experiments. (C) qRT-PCR analysis of cytokine transcript levels, as indicated, in bone marrow-derived macrophages, as induced by indicated stimuli after $48 \mathrm{~h}$ of stimulation. Data were normalized to Gapdh expression and expressed as the ratio to values of non-stimulated macrophages. Representative of two independent experiments. 

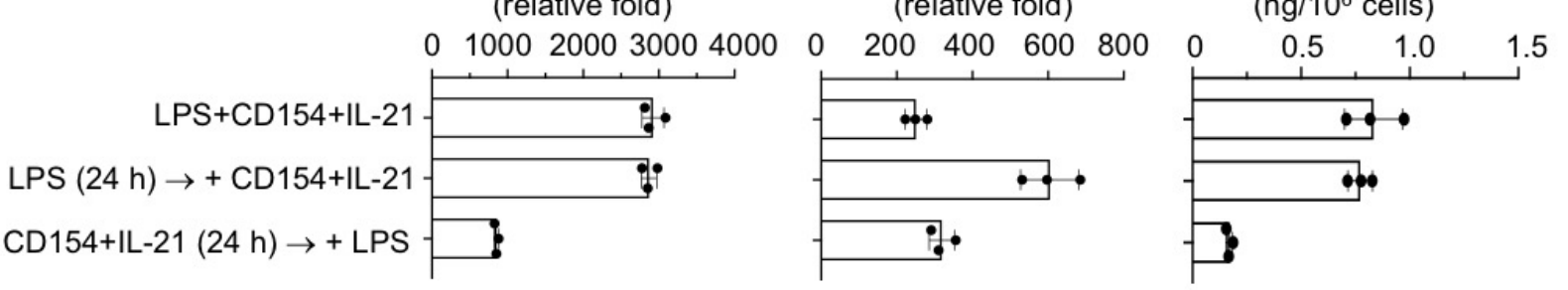

+TLR ligand
(0-48 hr)
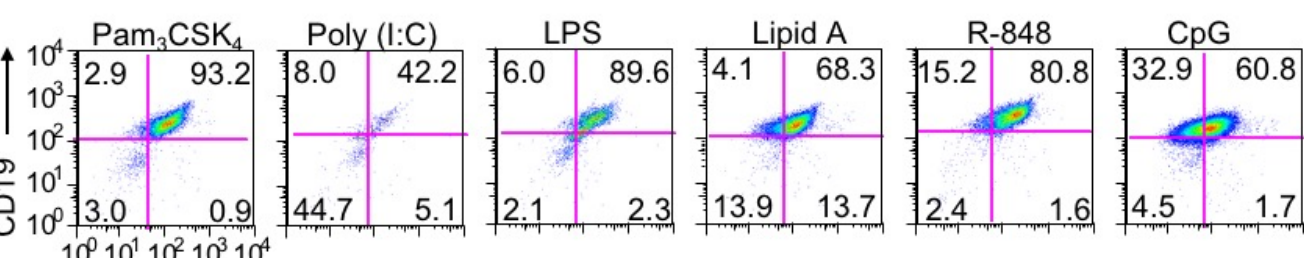
$10^{0} 10^{1} 10^{2} 10^{3} 10^{4}$

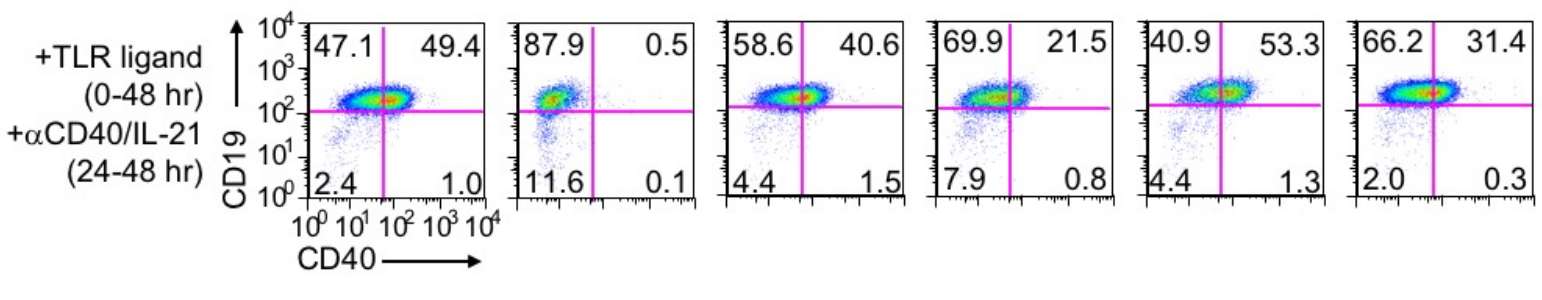

D

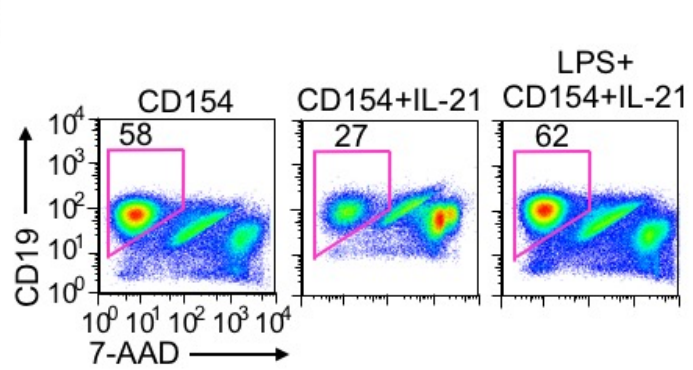

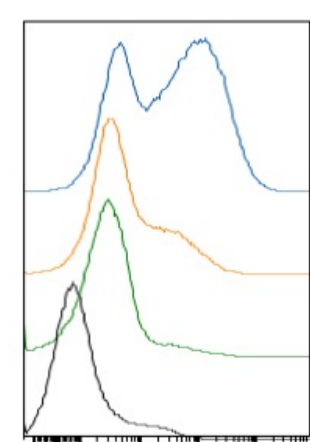

II27p28-GFP

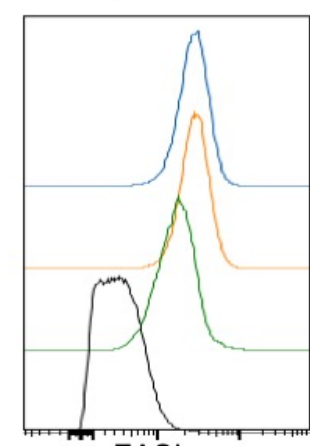

FASL

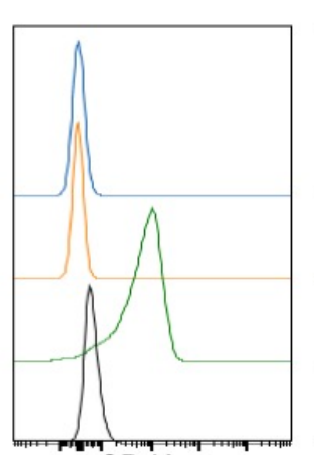

CD40

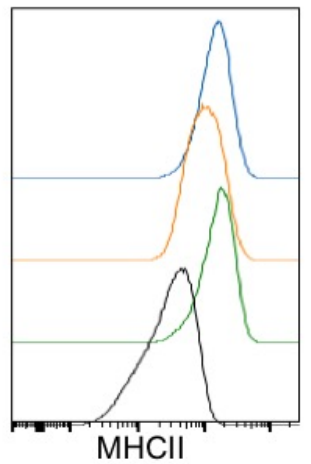

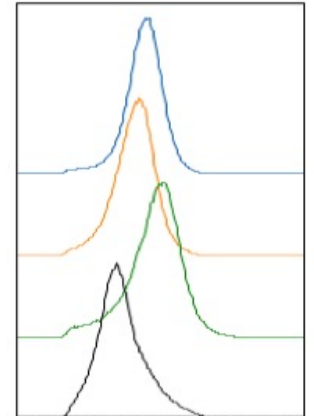

CD19

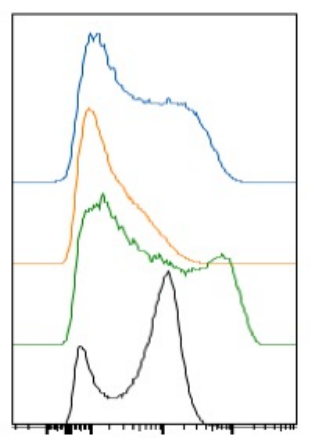

$\lg D$

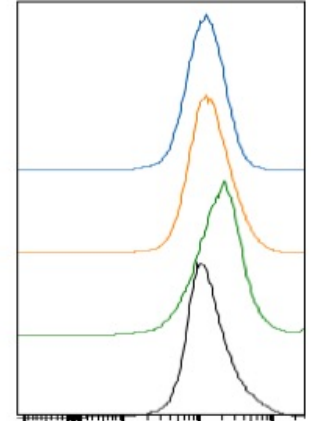

CD38

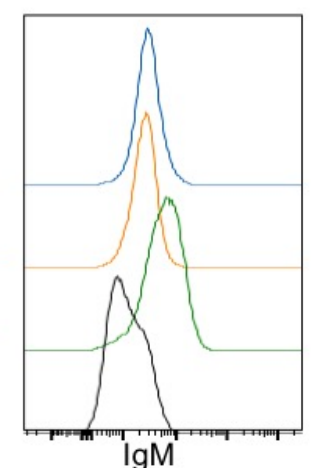

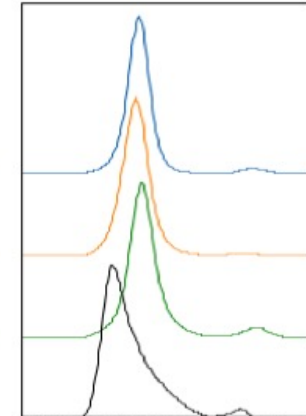

CD11b

$\square$ LPS+CD154+IL-21

$\square$ CD154+IL-21

$\square$ LPS

$\square \mathrm{Nil}$

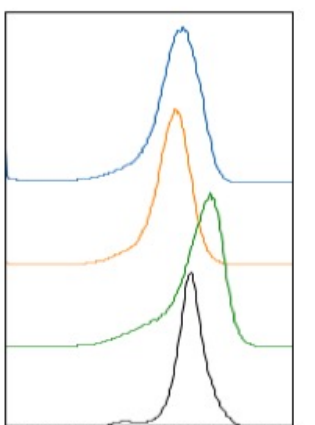

$\lg \kappa$

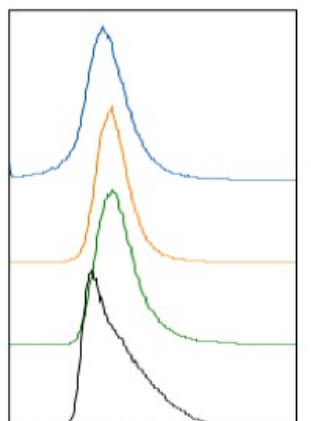

CD1d

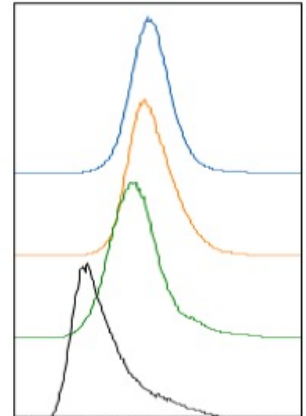

CD11c

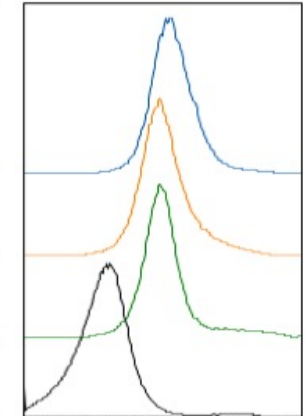

CD138

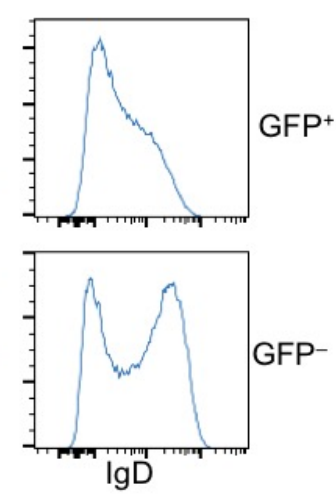

$\lg D$

fig. S3. Induction of IL-27 in B cells by sequential stimulation of LPS and CD154. (A) qRTPCR analysis of II27p28 and Ebi3 transcript levels (left two panels) and ELISA of IL-27 secreted into the supernatants (right panel) of purified spleen B cells after co-stimulation with LPS, CD154 or IL-21, or sequential stimulation, as indicated (mean and s.d., triplicates). (B) Flow cytometry analysis of cell viability (7-AAD-) in B cells stimulated, as indicated, for $48 \mathrm{~h}$. Representative of five independent experiments. (C) Flow cytometry analysis of CD40 surface expression in B cells stimulated, as indicated, for $24 \mathrm{~h}$. (D) Flow cytometry analysis of expression of CD40 and selected surface markers in B cell stimulated, as indicated, for $48 \mathrm{~h}$. Representative of two independent experiments. 
A

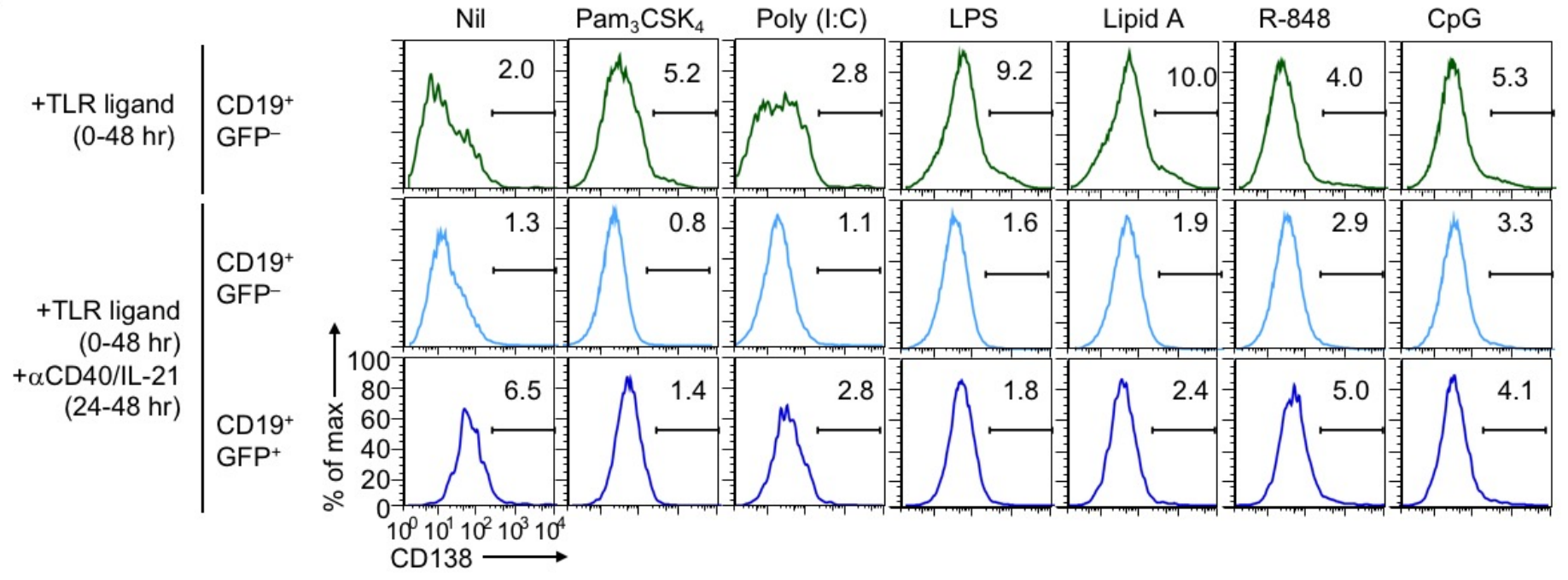

C

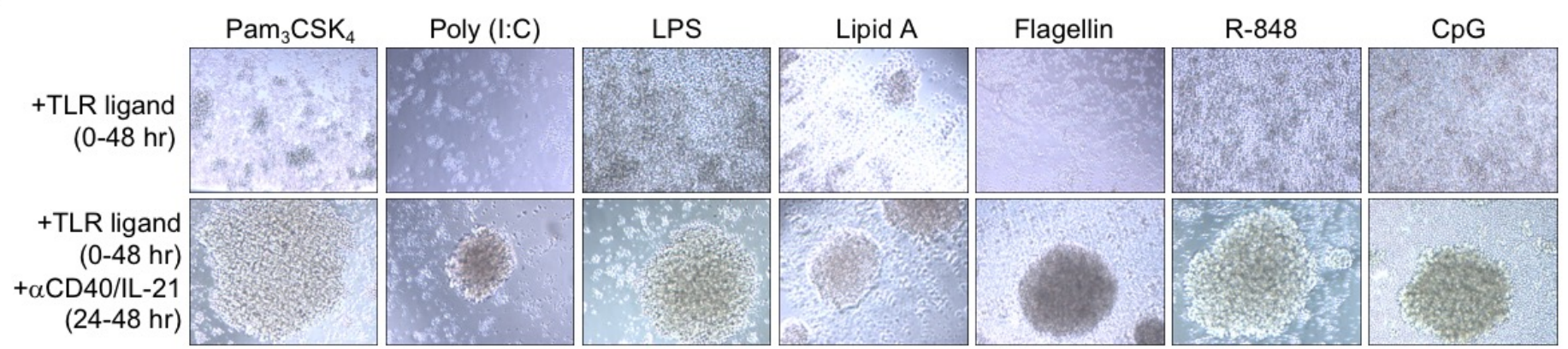

B

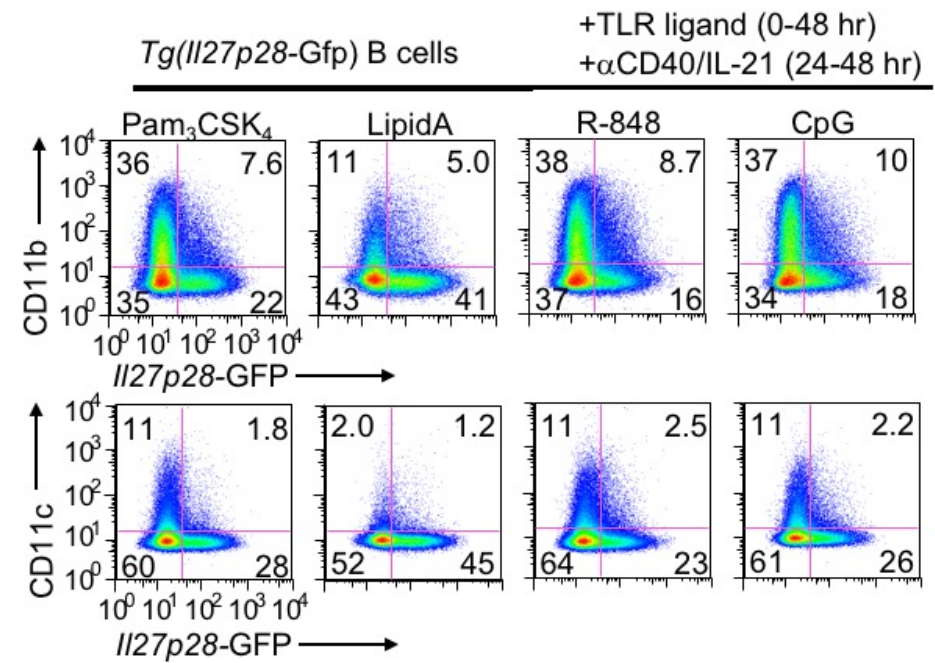

D

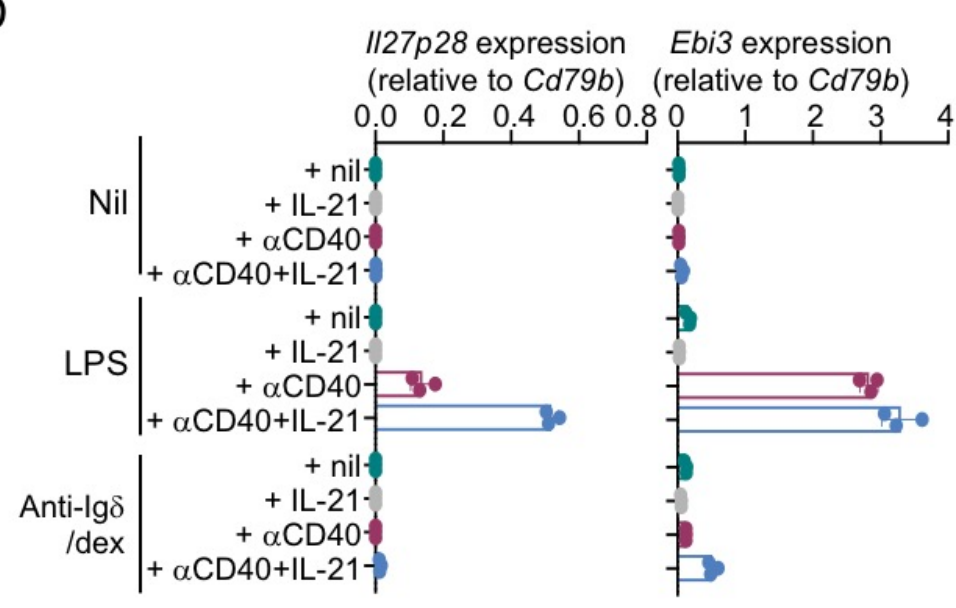

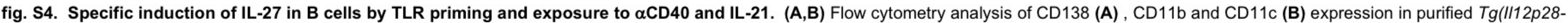

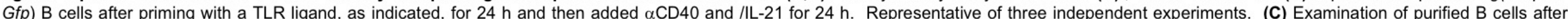

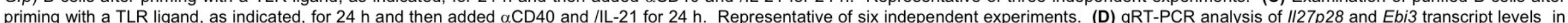

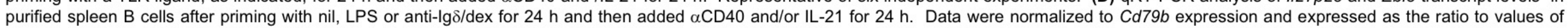
freshly isolated $B$ cells $(0 h)$. 
A

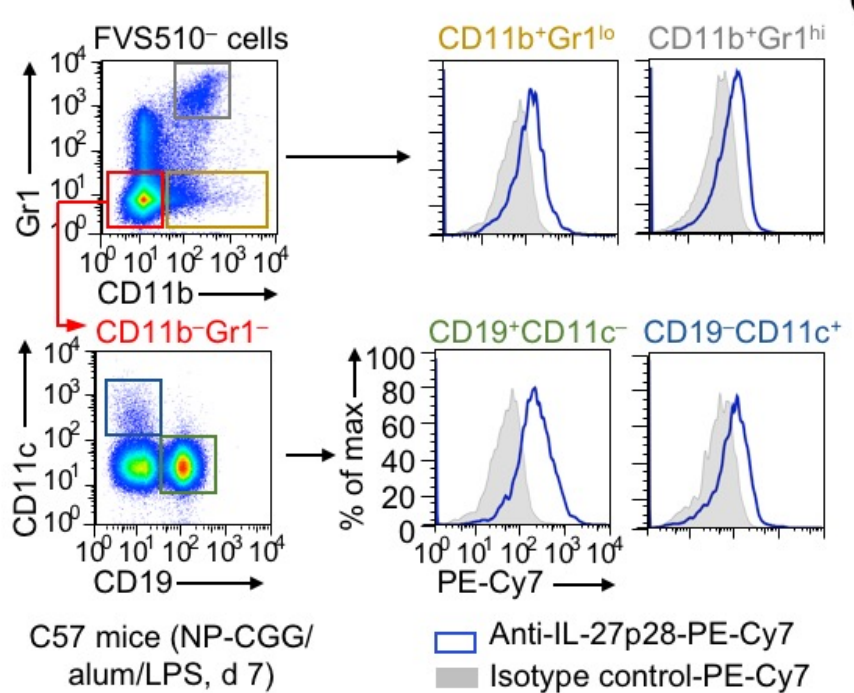

B

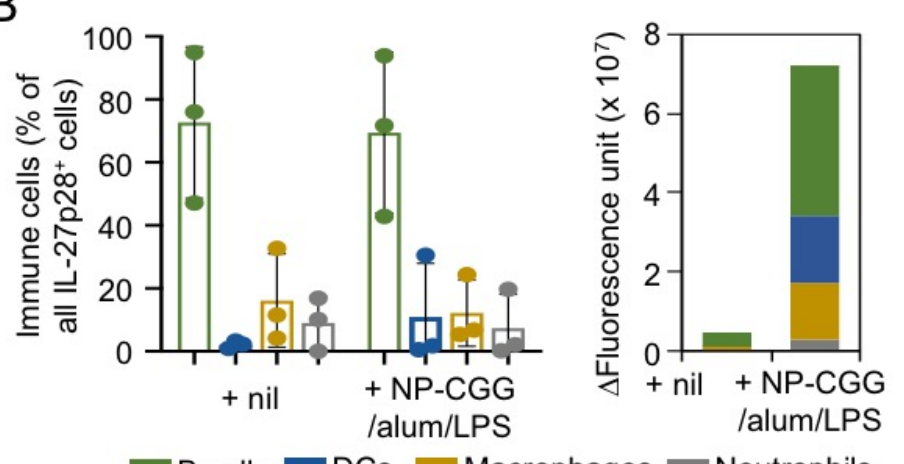

B cells
C
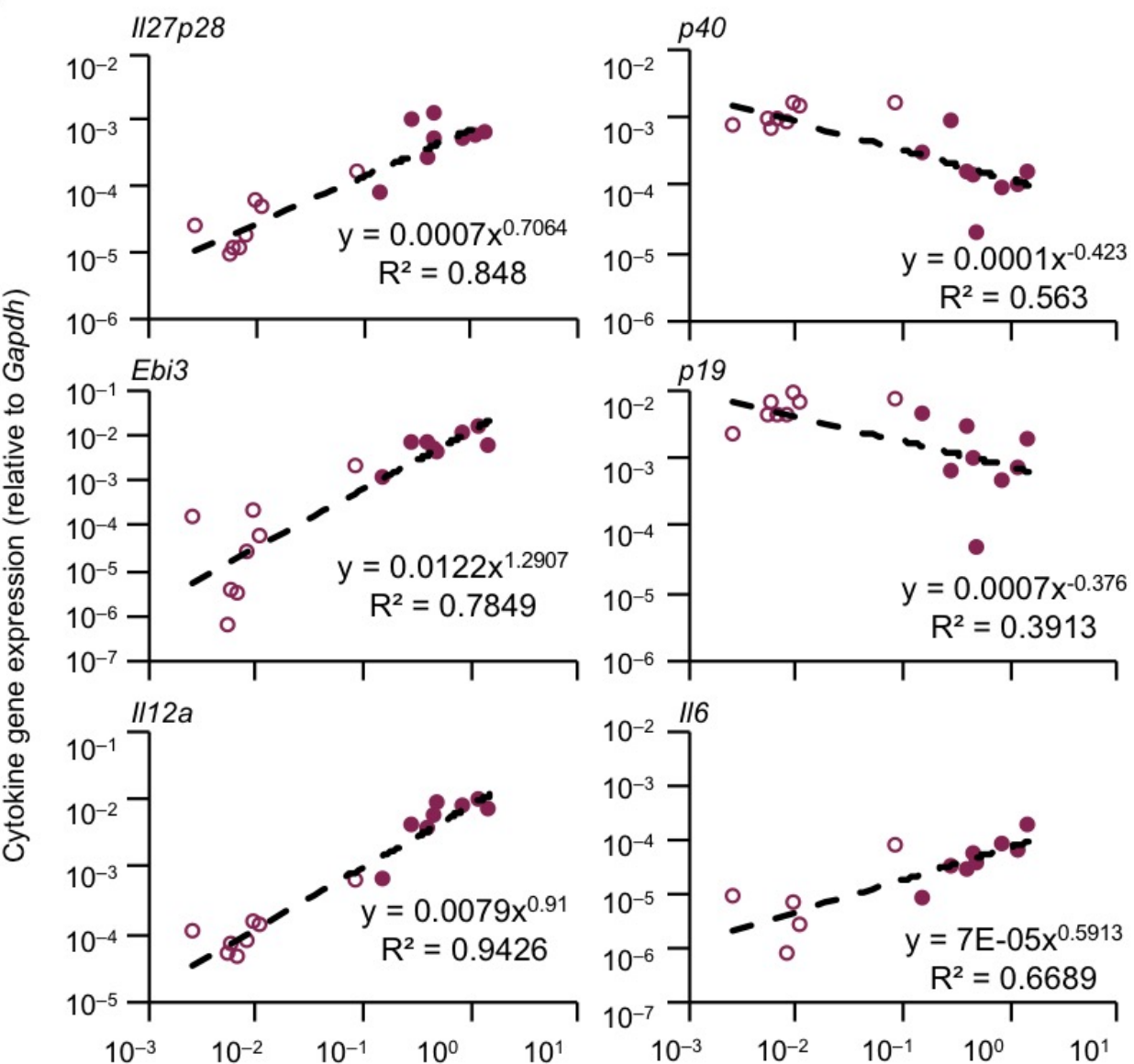

$10^{-2}$

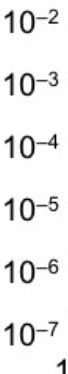

$\begin{aligned} & 0^{-3}- \\ & 0^{-4}- \\ & 0^{-5}- \\ & 0^{-6} \\ & 0^{-7}\end{aligned}-$

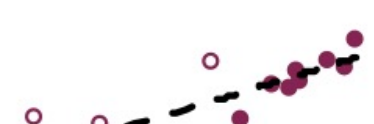

을 $y=7 E-05 x^{0.5913}$ $\mathrm{R}^{2}=0.6689$

Cd79b expressio

$10^{-3}$

3-month old

20-month old

fig. S5. Expression of IL-27 by B cells in vivo. (A) Intracellular staining with a IL-27p28-specific Ab and isotype-matched IgG control Ab and flow

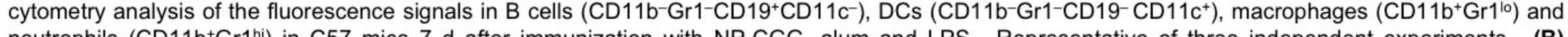
neutrophils $\left(C D 11 b^{+} G r 1{ }^{\text {hi }}\right)$ in C57 mice $7 \mathrm{~d}$ after immunization with NP-CGG, alum and LPS. Representative of three independent experiments. (B) Proportions of different immune cell populations, as defined in (A), in IL-27p28+ cells, as analyzed by flow cytometry, in C57 mice before or $7 \mathrm{~d}$ after immunization (left panel); also depicted is the cumulative fluorescence unit of each population, as calculated by multiplication of the number of cells with the subtraction of fluorescence arising from isotype-matched IgG Ab staining from that of anti-IL-27p28 staining (right panel). (C) Correlation analysis of transcript levels of cytokine-encoding genes, as indicated, with that of Cd79b, as determined by qRT-PCR, in 8 young (3-month old) and 8 aging (20-month old) mice that were not immunized. Data were normalized to Gapdh expression for the calculation of power regression and $R^{2}$ value (Excel). 
A
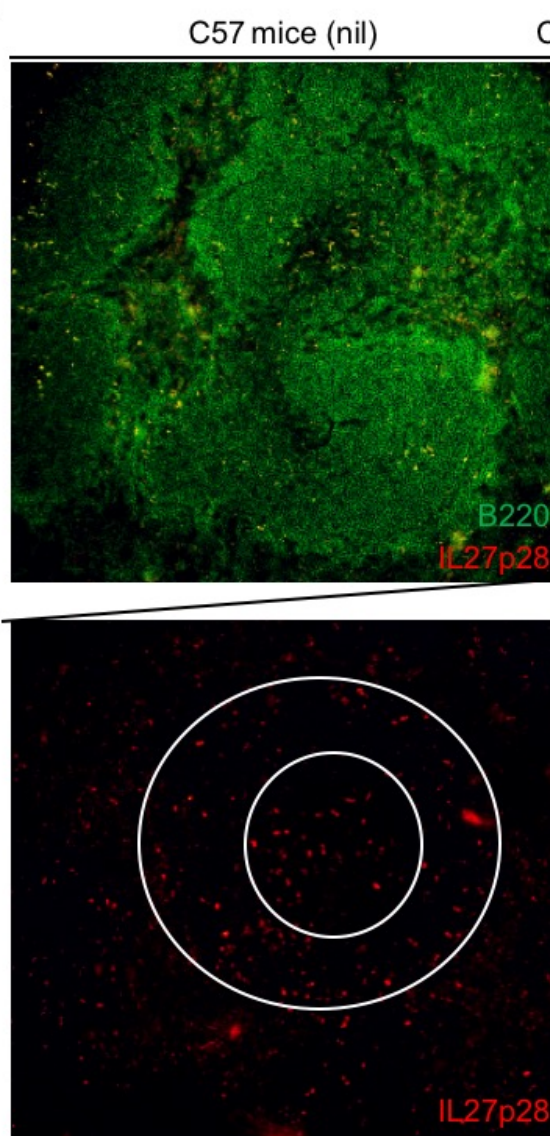

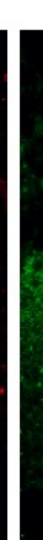

C57 mice (NP-CGG/alum/LPS, spleen, d 7)
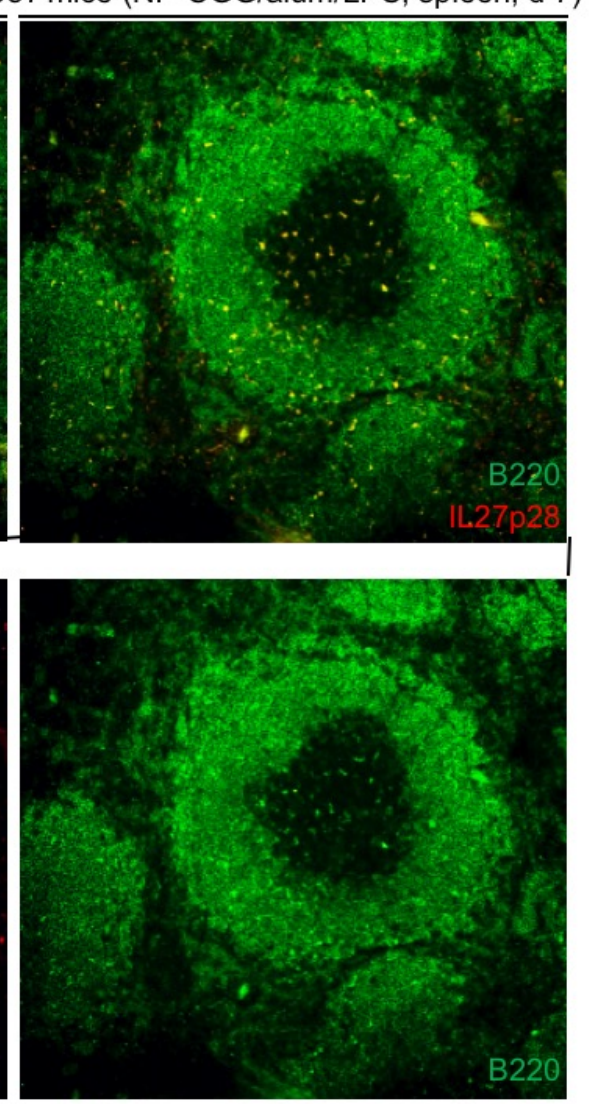

\section{B}

II110IRES-GIP mice

(NP-CGG/alum/LPS, d 7)
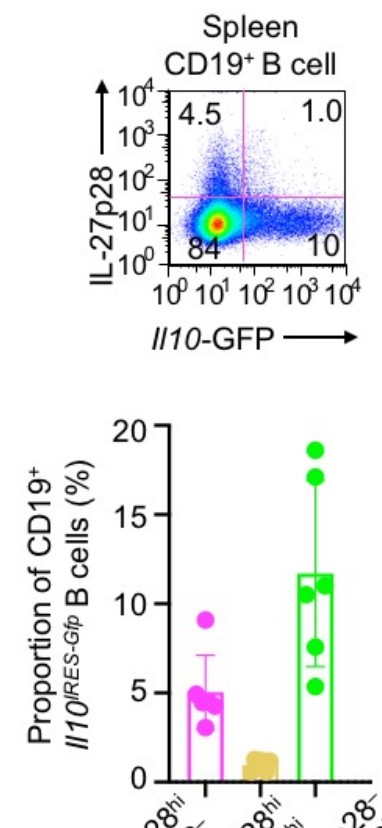

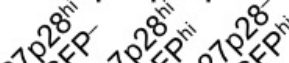

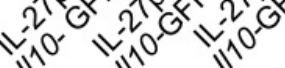

fig. S6. IL-27-producing B cells in mice upon immunization. (A) Immunofluorescence microscopy of IL$27 \mathrm{p} 28^{+} \mathrm{B} 220_{+}$cells in the spleen of $\mathrm{C} 57$ mice after immunization. A representative follicle in the filed is shown. (B) Flow cytometry analysis of IL-27p28 and GFP (indicating //10 transcription) in B cells in immunized I/10 IRES-Gtp mice. Data quantification (lower panel) are from three independent experiments. 
A

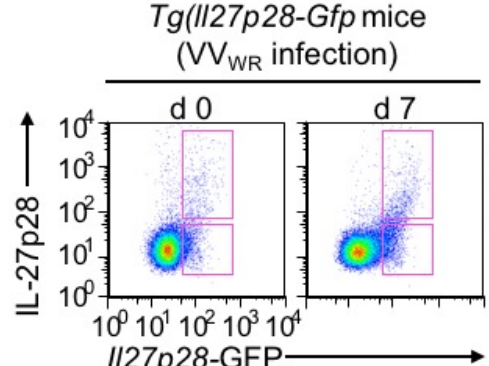

B

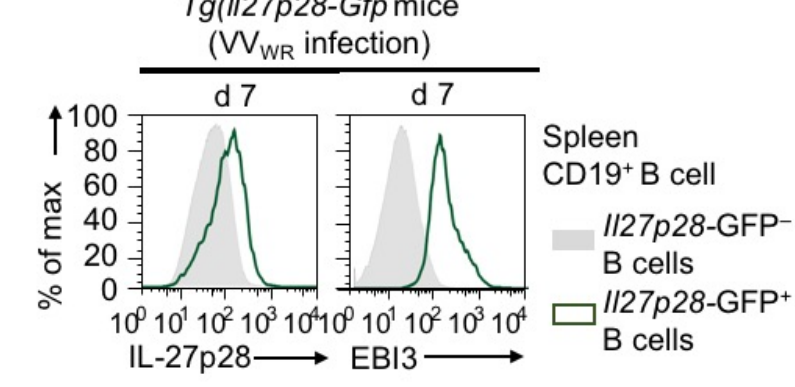

C II10/RES-GIp mice $\left(V_{\text {WR }}\right.$ infection, $\left.d 7\right)$ $\mathrm{D}$ Mediastinal lymph

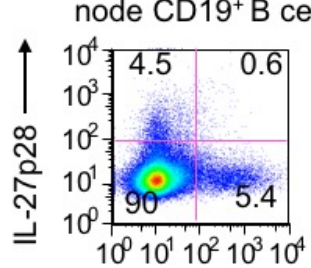
I10-GFP

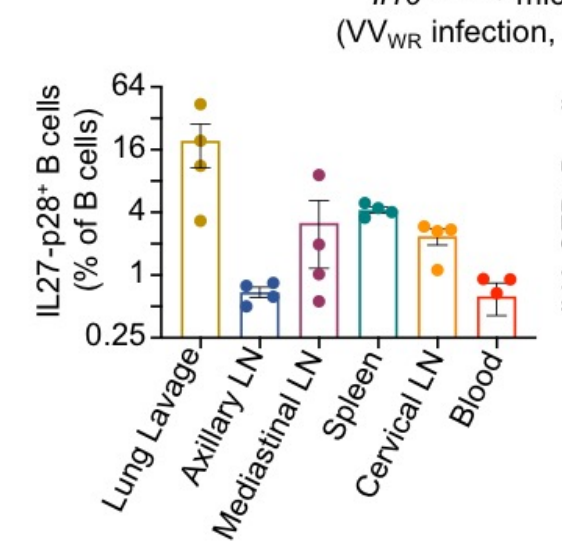

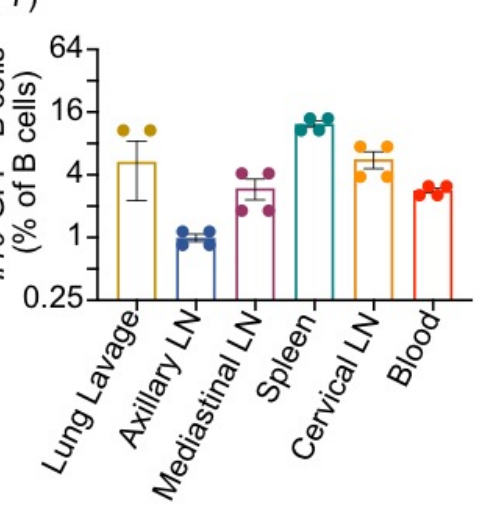

fig. S7. IL-27-producing $B$ cells are induced in mice upon $\mathrm{VV}_{\mathrm{WR}}$ infection. (A) Flow cytometry analysis of IL-27p28 and GFP (indicating I/27p28 transcription) expression to quantify IL-27p28 induction in mediastinal lymph node $B$ cells $T g$ (II27p28-Gfp) mice before and after infection with $V V_{W R}$ infected. (B) Flow cytometryanalysis of IL-27p28 and EBI3 in infected Tg(II27p28-Gfp) mice. Representative of three independent experiments. (C,D) Flow cytometry analysis of IL-27p28 and GFP (indicating $/ 110$ transcription) expression (C, representative flow cytometry plot) to quantify IL-27p28 protein and I/10 transcription induction in infected $/ / 10^{R E S S-G f p}$ mice (D, and quantification of data from different tissues and organs). 
A

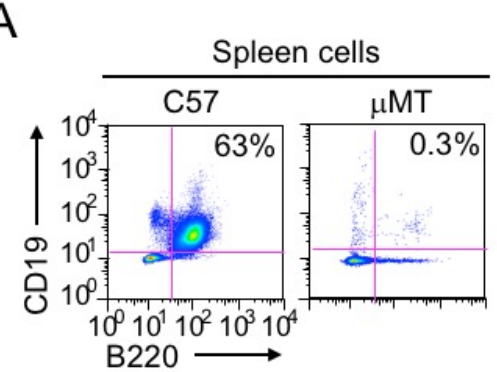

B

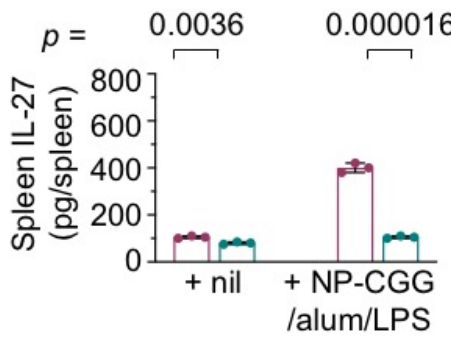

C

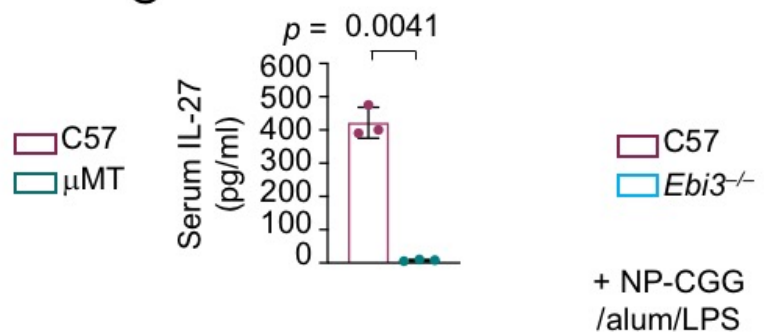

fig. S8. Reduced IL-27 production in mice lacking B cells. (A) Flow cytometry analysis of lack of B cells in $\mu$ MT mice. (B) ELISA of IL-27 in the spleen in C57 and $\mu$ MT mice before and after (d 7) of immunization. (C) ELISA of IL-27 in the circulation of $\mathrm{C} 57$ and $\mathrm{Ebi}^{-/}$mice after immunization for $7 \mathrm{~d}$. 
A frt

loxP

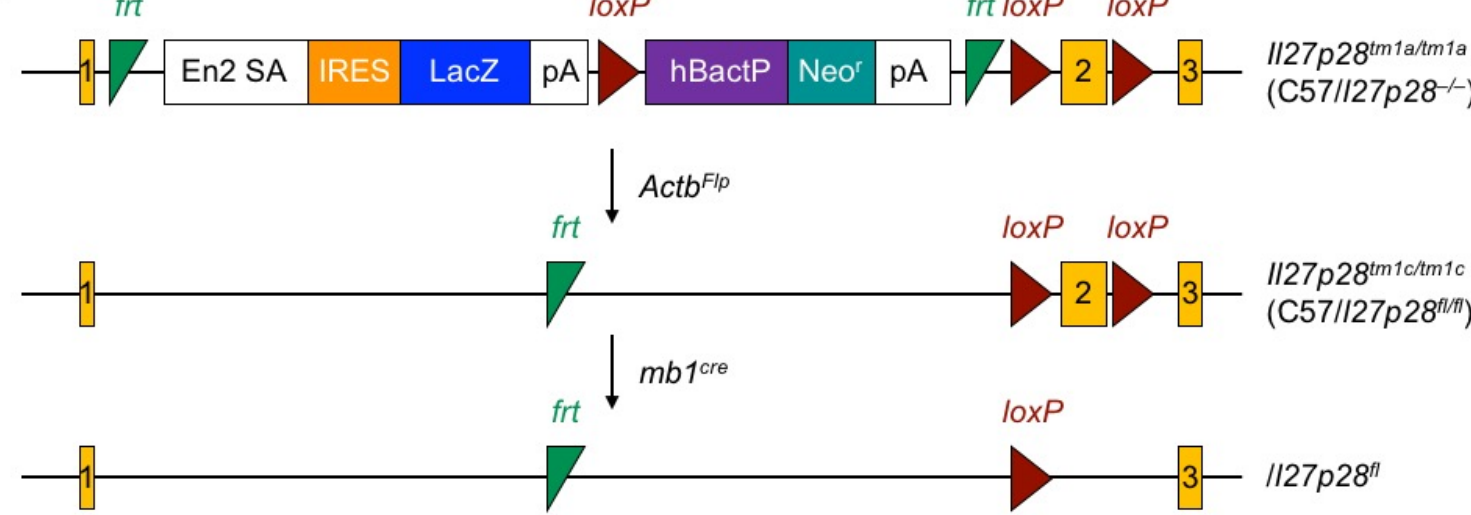

$B$
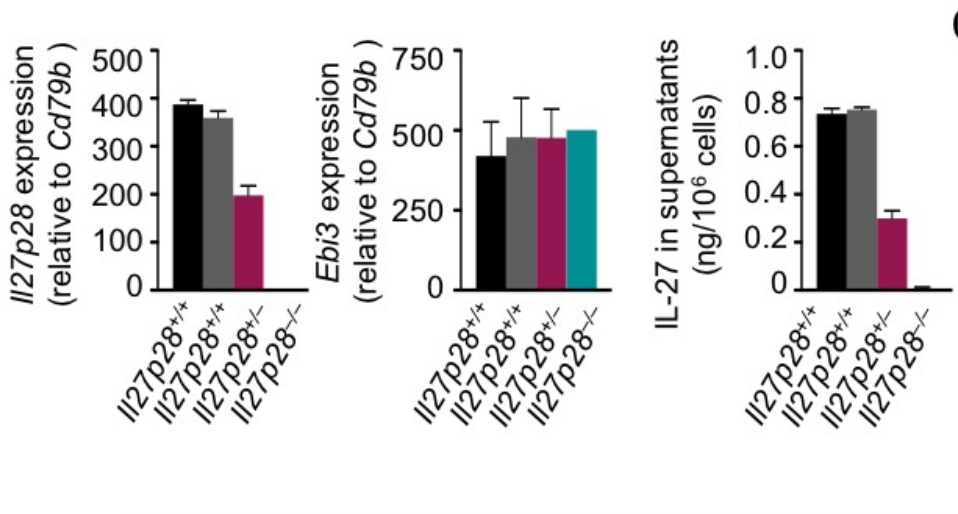

C

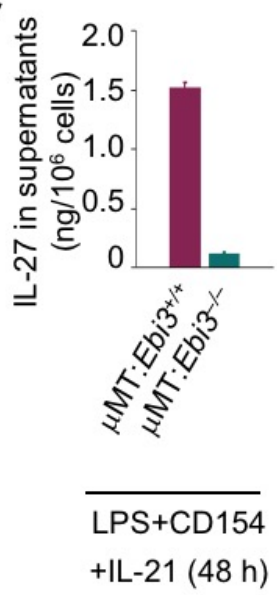

LPS+CD154

+IL-21 (48 h)
D

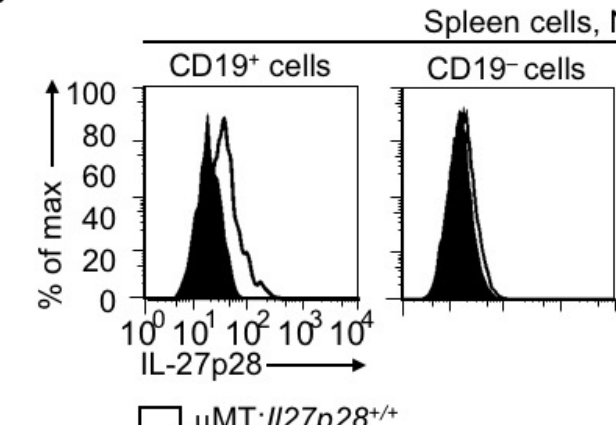

$\square \mu \mathrm{MT}: / / 27 p 28^{+/+}$

- $\mu \mathrm{MT}: / / 27 p 28^{-\alpha}$
Spleen cells, NP-CGG/ alum/LPS (d 7 )

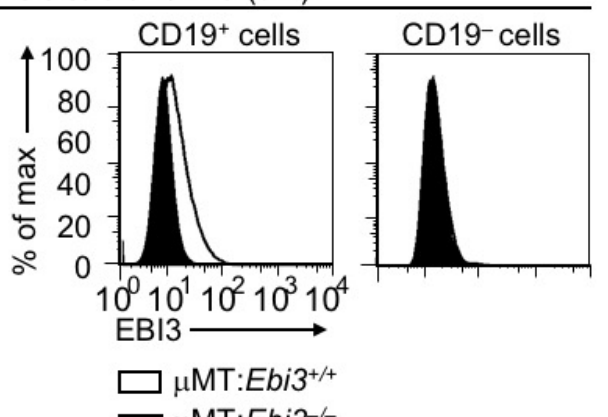

CD19+ cells, NP-CGG/ alum/LPS (d 7)

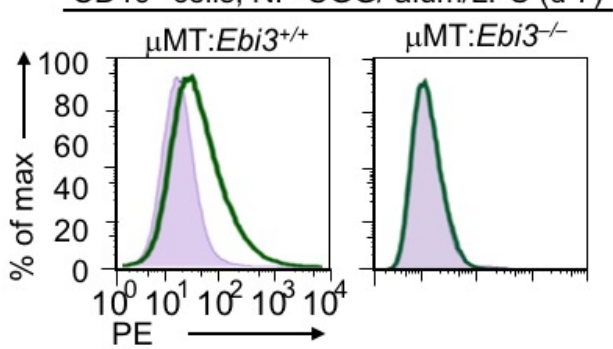

PE-isotype control

$\square$ PE-anti-EBI3

fig. S9. B cell-specific deficiency in II27p28 or Ebi3 results in decreased IL-27 production. (A) Schematics of II27p28- allele, due to the interference of II27p28 transcription from a large insert between exon 1 and exon 2 in the $1 / 27 p 28^{t m 1 a}$ allele, and the generation of $1 / 27 p 28^{f l}$ allele, which is independent from that used in this study. (B) qRT-PCR analysis (left) and ELISA (right) for the confirmation of lack of $1 / 27 p 28$ transcription and IL-27 secretion in $1 / 27 p 28^{-1}$ B cells stimulated in vitro. qRT-PCR Data were normalized to Cd79b expression and expressed as the ratio to values of freshly isolated B cells $(0 \mathrm{~h})$. (C) ELISA for the confirmation of lack of IL-27 secretion in $\mu \mathrm{MT}$ :Ebi3 ${ }^{-\sim} \mathrm{B}$ cells stimulated in vitro. (D) Intracellular staining and flow cytometry analysis for the confirmation of lack of IL-27p28 and EBI3 expression in immunized $\mu \mathrm{MT}: / / 27 p 28^{-/}$and $\mu \mathrm{MT}: E b i 3^{-/}$mice, respectively. Representative of two independent experiments. (E) ELISA of reduced IL-27 in the circulation in $\mu \mathrm{MT}: E b 3^{-}-$mice at different timepoints after immunization. 

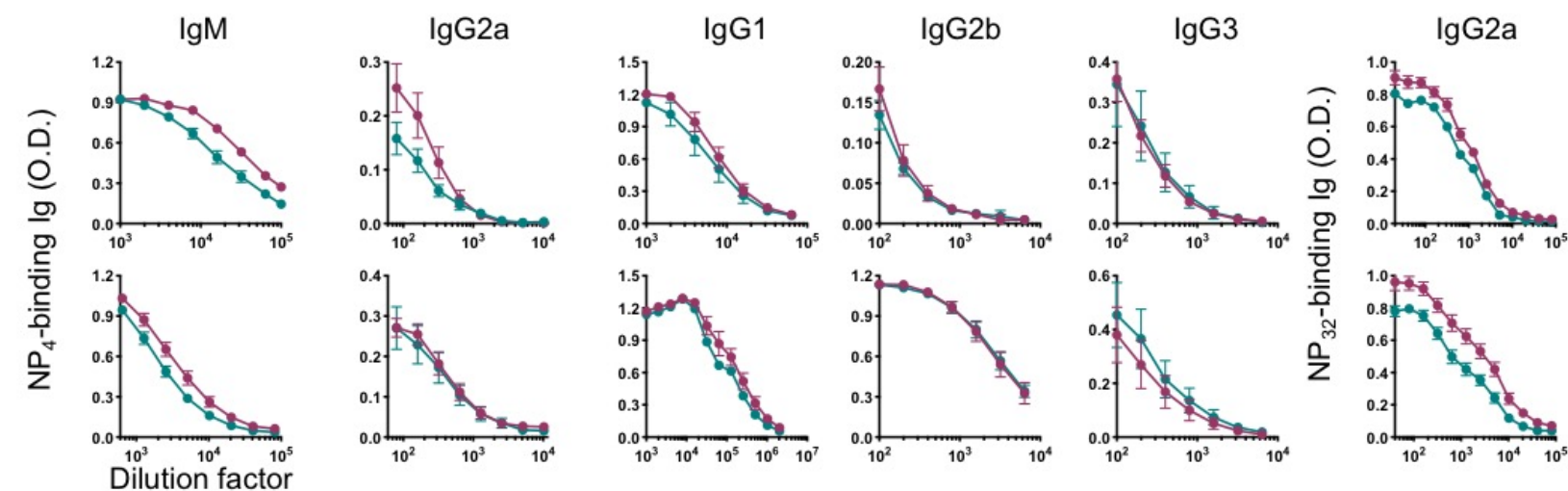

: 1.00 㱒

$\stackrel{\infty}{2}^{\infty}$

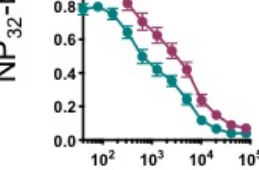

B

$\rightarrow \mu \mathrm{MT}: E b i^{+/+}(\mathrm{n}=4) \quad \sim \mu \mathrm{MT}: E b i^{-} \sim(\mathrm{n}=4)$ $\lg M$
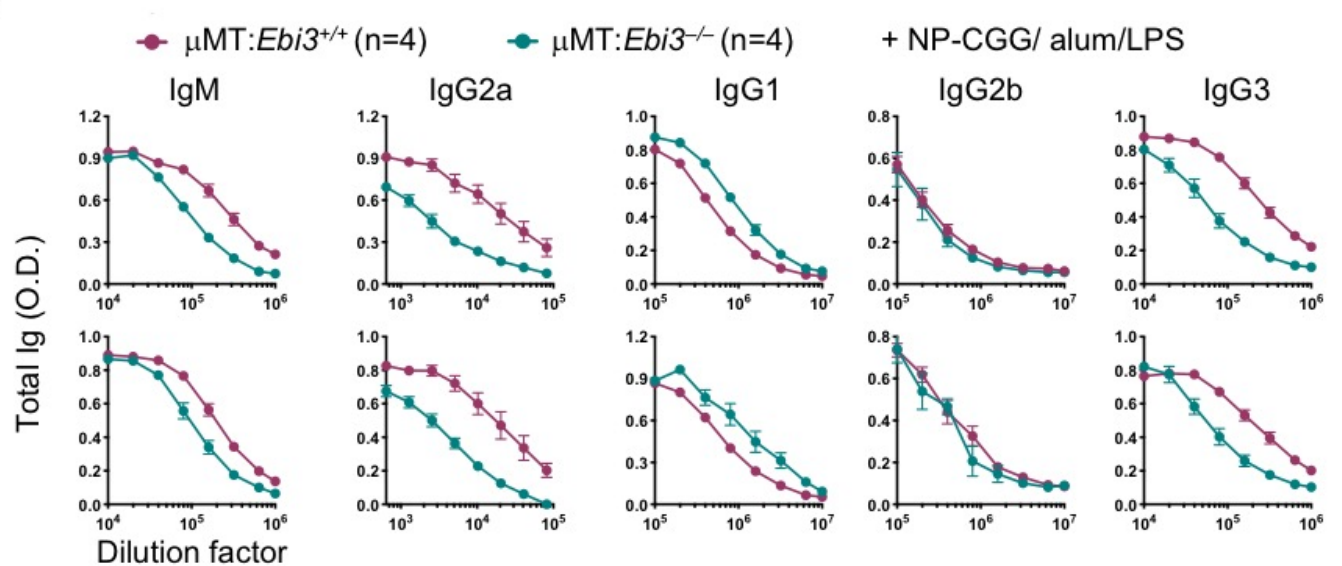

D
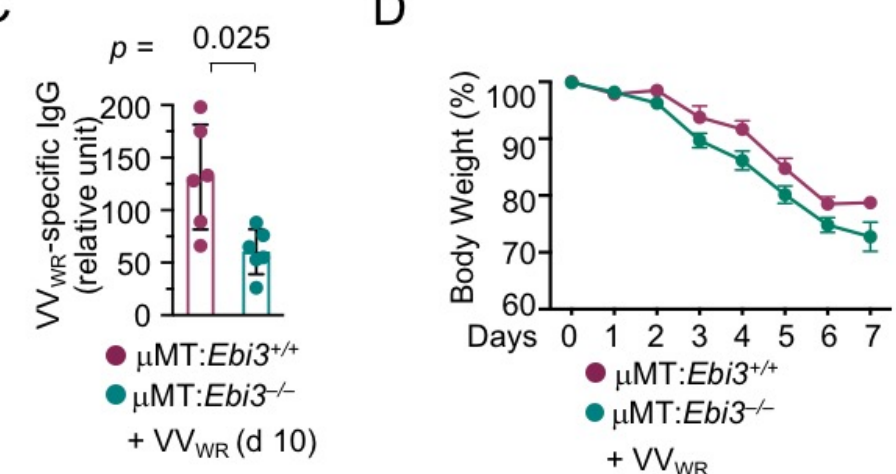

fig. S10. Defective IgG2a responses in $\mu \mathrm{MT}$ :Ebi3-- mice.

(A) ELISA of $\mathrm{NP}_{4}$-binding (high-affinity) and (all specific)

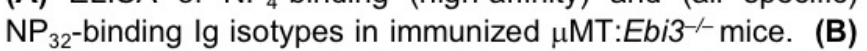
ELISA of total Abs of different Ig isotypes in immunized $\mathrm{VV}_{\text {WR-infected } \mu \mathrm{MT} \cdot \mathrm{Ebi3}^{-/} \text {mice, as in (A) (C,D) ELISA of }}$

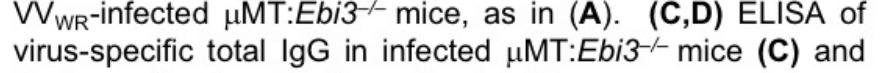
the loss of body weight in such mice (D). 
A

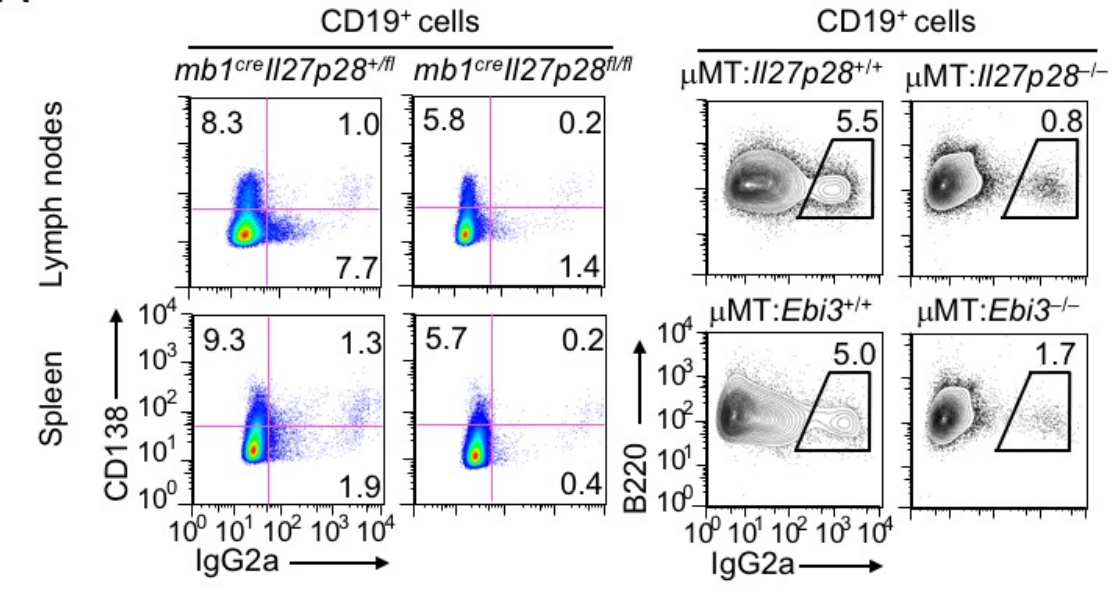

fig. S11. Impairment in CSR to IgG2a in mice with B cell-specific deficiency in $1127 p 28$ or Ebi3. (A) Flow cytometry analysis of IgG2a and/or CD138 in spleen and lymph node B cells in immunized

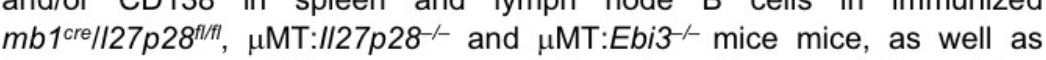
their respective wildtype mouse counterparts. Representative of two independent experiments. (B) Flow cytometry analysis of cell viability, CD138 and GL-7 in immunized mice, as in (A). Representative of three independent experiments.
B
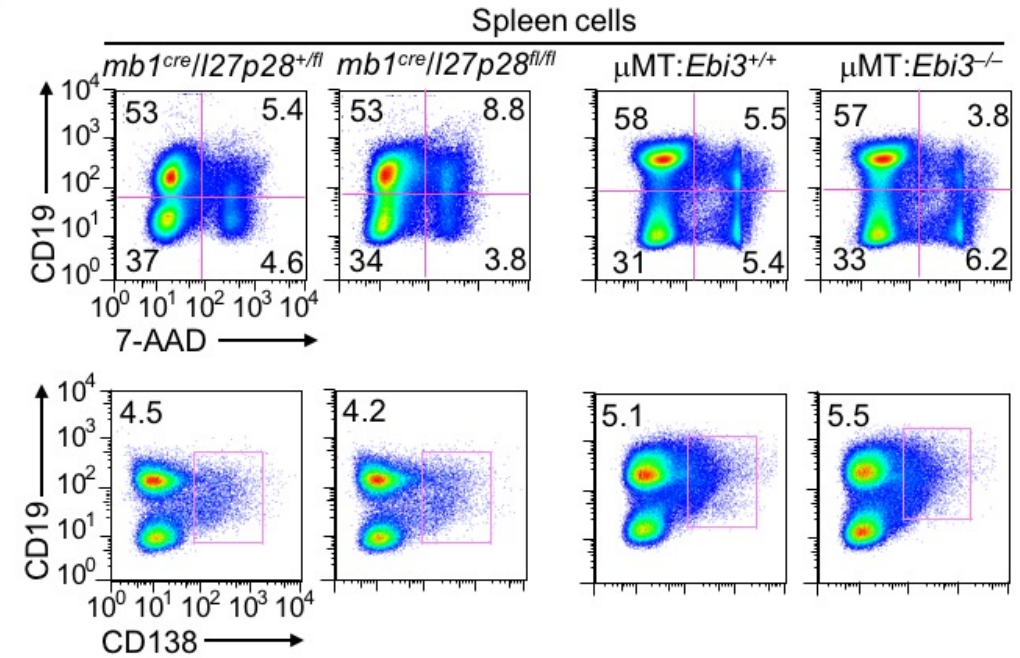

$10^{4} \sqrt[3]{3.2}$
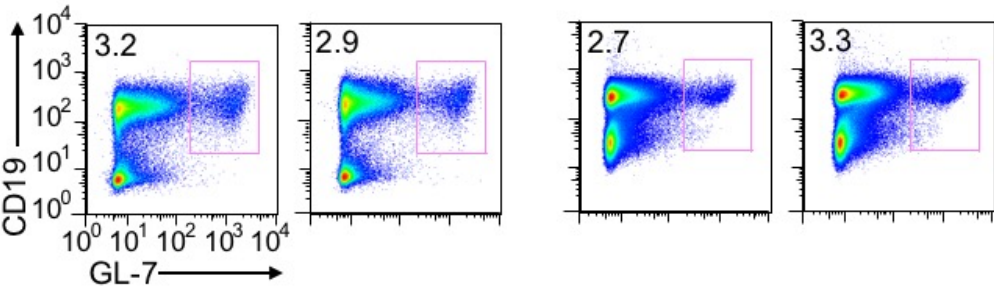
A

LPS + CD154+IL-21 vs LPS

DA proxima

$\begin{array}{cc}\text { DEGs } & \text { regions } \\ \text { (RNA-Seq) } & \text { (ATAC-Seq) }\end{array}$

C
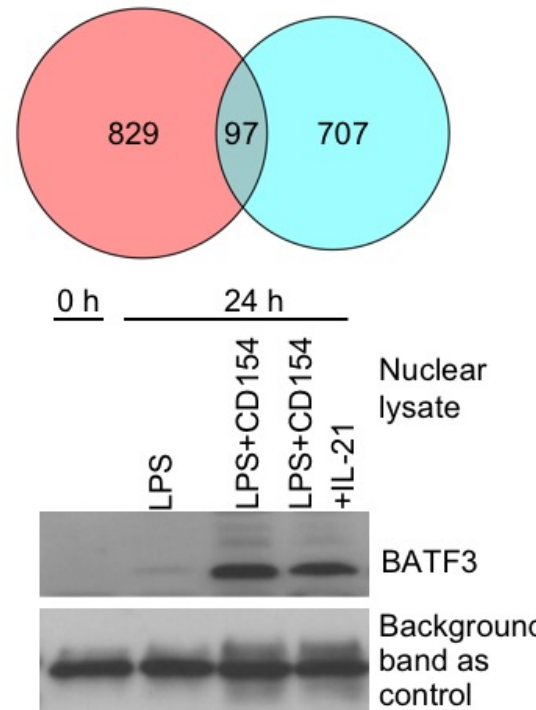

B

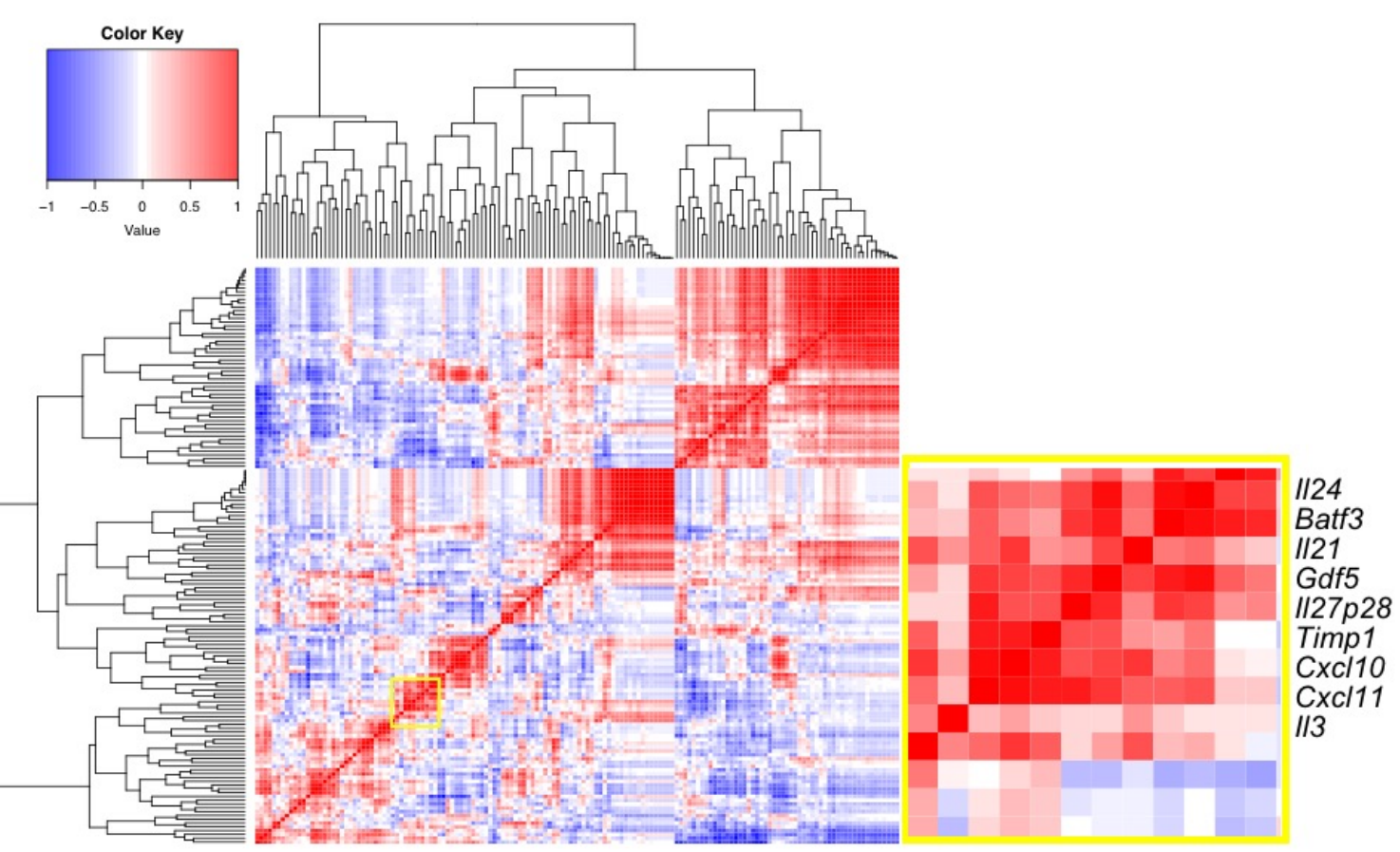

fig. S12. BATF3 is upregulated in B cells induced to express IL-27 in vitro. (A) Venn diagram of 97 genes that had DARs and DEGs in B cells stimulated with LPS plus CD154 and IL-21 to induce IL-27, as compared to LPS-stimulated B cells. II27p28 is one of the 97 genes. (B) Correlation analysis of the expression level of of Batf3 and that of 120 expressed cytokine and chemokine-encoding genes in duplicate $B$ cells stimulated in vitro with nil (0 h), (CSR-inducing) LPS, CD154 and CD154 plus IL-4, as well as (IL-27-inducing) LPS plus CD154, CD154 and IL-21, and LPS plus CD154 and IL-4, for $24 \mathrm{~h}$. (C) Immunoblotting of induced BATF3 in the nucleus of B cells stimulated with LPS plus CD154 and/or IL-21. Representative of two independent experiments. 


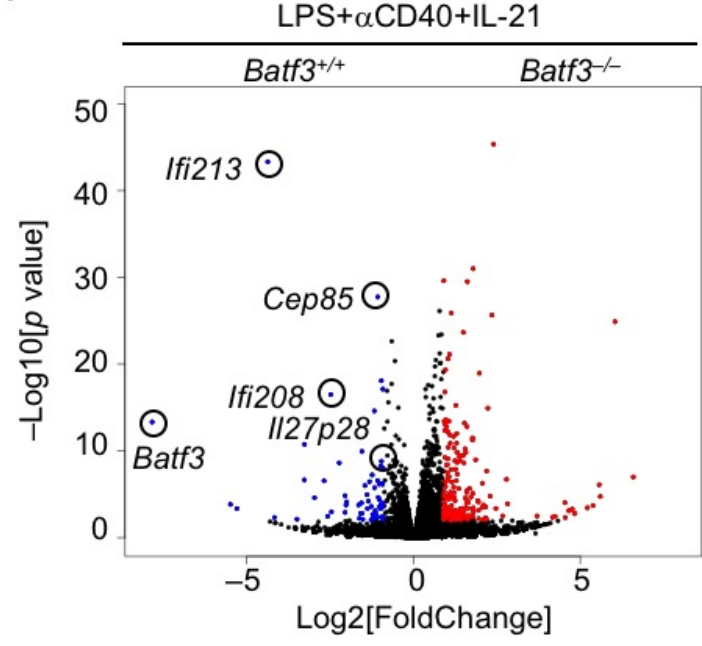

Positive regulation of cytokine production Positive regulation of
defense response Response to Response to
IFN $\beta$ Cellular response to IFN $\beta$ Response to
IFN $\gamma$

Response to

virus
Cellular response
to IFN $\gamma$ Defense response to protozoan

Response to protozoan

Interleukin-1 $\beta$ production

fig. S13. BATF3 mediates IL-27 induction in $B$ cells in vitro. (A) Volcano plot DEGs in Batf $3^{-/}$and Bat/3 $B$ cells after stimulation for 48 h. (B) GO analysis of DEGs in stimulated Batf $3^{--}$and $\mathrm{Batf}^{+/+} \mathrm{B}$ cells, as in (A). (C) Lack of significant DARs, as determined by ATAC-Seq, in Batf3 $3^{--}$and $\mathrm{Batf}^{+/+} \mathrm{B}$ cells after stimulation with LPS plus $\alpha \mathrm{CD} 40$ and IL-21 for $48 \mathrm{~h}$.
C

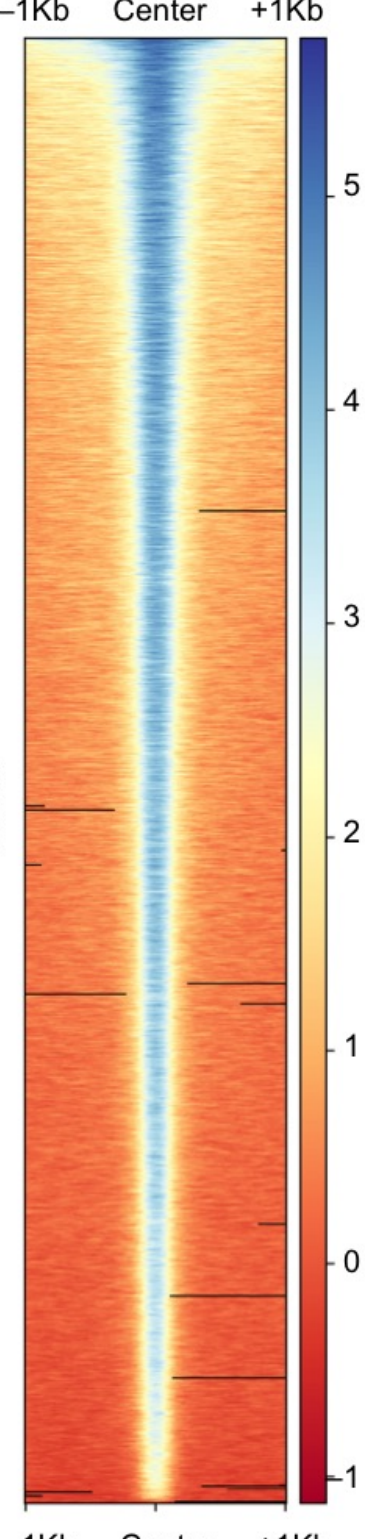


A

Spleen cells

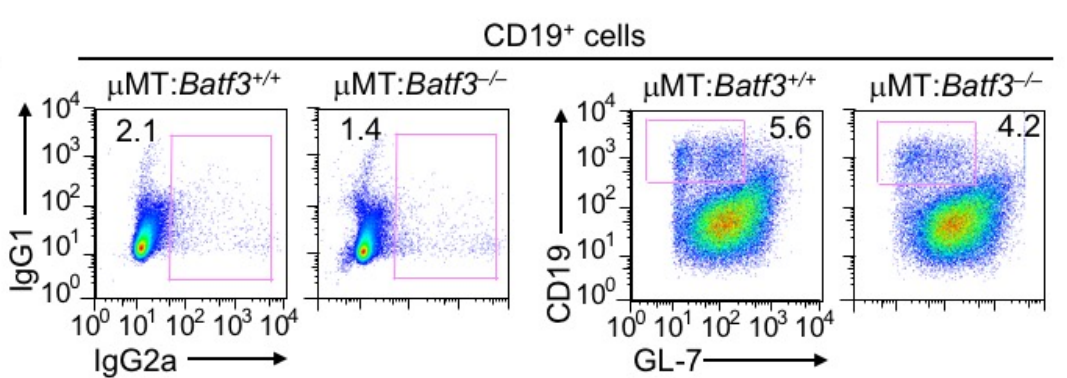

CDigt cells
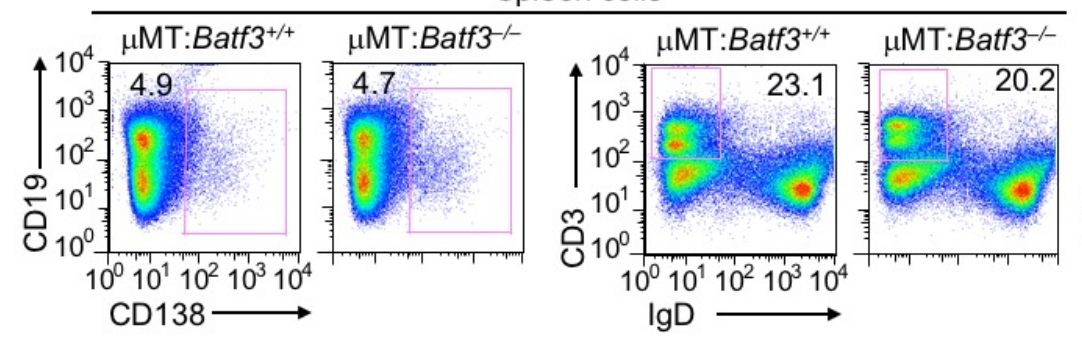

$\lg \mathrm{G} 2 \mathrm{a}$

B

$$
p=0.17
$$

$p=0.023$

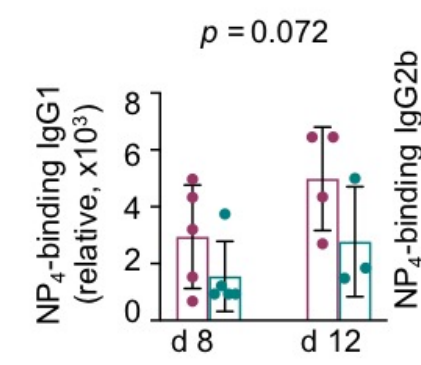

$p=0.097$

$p=0.21$
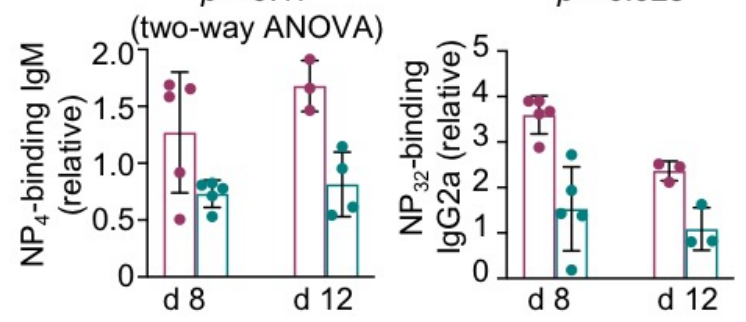

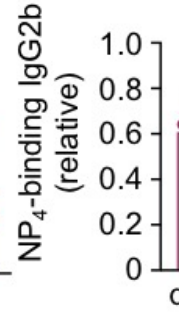

p.
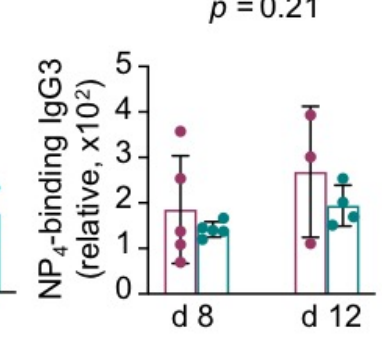

口 $\mu \mathrm{MT}:$ Batf3 $^{+/+}$ $\square \mathrm{MT}:$ Batf3- $^{-}$ + NP-CGG/alum /LPS (d 12)

fig. S14. B cell-intrinsic role of BATF3 in mediating IL-27 induction and an antigen-specific IgG2a response. (A) Flow cytometry analysis of B and T cell proportions in the spleen (left) as well as B cell CSR and germinal center differentiation (right) in $\mu \mathrm{MT}:$ Batf3 $\_$mice and their wildtype counterparts immunized with NP-CGG plus alum and LPS for $12 \mathrm{~d}$. Representative of three independent experiments. (B) ELISA of NP-specific Ig isotypes in $\mu$ MT:Batf3 -mice and their wildtype counterparts immunized with NP-CGG plus alum and LPS for 8 and $12 \mathrm{~d}$. 
GTAGCCCGCTCCCTCTTCCTCCTCCTTTGAAGATAAGATCGAACCATGCAGCTCTGGCTACCTGTGACCTCACAAGACACATTCCTACCTTACTC TCCTTGCCGGGCTTGTGGGCACCACTGGACCTGTGGACTTGCTATTATGTAACAATCACCCTACAAGAGACTCTCCCGTTCCTGGAGGACTGTCA CAGCTCCCTTATTCTCTTTCTTAGACGTGAGTTACAACGTCTAGTCTGTCCCTGTCACTGTCTGCAATGTGCAGAGAGAACTGTAGCTTAGGCCT TATGGCCCTGTCACACTCAAGAGGACTGACTACAGTCAAAGGATGCAAATAAGCCGGGCGTGGTGGCACACGCCTTTAAATCCCAGCACTTGGGA

GCAGAGGCAGGCGGATTTCTGAGTTCAAGGCCAGCCTGGTCTACAGAGTGACTTCCAGGACAGCCAGGGCTACACAGAGAAACCCTGTCTTGAA AACCAAAAAAAAAAAAAAAAAAAAAAAAAGGATGCAAATAGCCTGGCTTCTGAGGTGGCACCAATGCTTAAATGAGGTGGGGAAAAGAGAGAGGA AACCAAAAAAAAAAAAAAAAAAAAAAAAAGGATGCAAATAGCCTGGCTTCTGAGGTGGCACCAATGCTTAAATGAGGTGGGGAAAAGAGAGAGGA AGTCACCACACCACTATTTAGATAGGAATGAAGTTTGAAATGACTACTCTATAACACAGAAACAATGACTAATAAGCCAGTGAACATTGAAGCAA TATCTGGCCAGCACAGGACATAACAGCCATTCAGGACGCTCTACCCCCACTCTGACAATGACCCATGTTTGTAGGCTGCAATGCAATTCACAGCA CTGAGCACCGAGGTTATAAGCACGAGCCACAAGAACAGCTGGCCATGGAATTAAGGGATTCCCATGAAGT

$$
\text { AP-1 site }
$$$$
\text { Composite AP-1/KB site }
$$

GAGGAGGAAGGGGGTGGCTTTTTGTGTCAAAGTCTTACATGGAGCCAAAGTGAACCTGGAACTAGCCATCCTCCTGCCCCAGCCTCCTGGGTGAT AGCATCCCAGGTGGGTGCCTCCATGCTCAGTAACAGTAAATTCTTTGGCTGCCTGGAGTTTTCTGGGGCAGGCACTGTAAGTGGAGATGGGGGAA CATTAGGGATCATAACCTGAGCTGCTAGAAGCTTAGTGTTGGCTCAGATCTTTGTAAGTTGGGTTCCAGTGGAGAAATGGCTCAGAGGCTGGGAT

Enhancer II TTGGTTTCCTCCCAGCTCAGGTGAAAAGATCAGAGGTCAGGGTCACTCCTCCGAGTGCTCCATCCTCTCACTGAACTCCAGGTCTGTGCCCTCTI TGTTCAGGACAGCCTATATTAGGGTGTTTGTGTCCACATCTGCCCTGTGAAGGAAGTAAGAAGTGGTATCAGAATGACTTTGTGGTTTCTATTGG TGTTCAGGACAGCCTATATTAGGGTGTTTGTGTCCACATCTGCCCTGTGAAGGAAGTAAGAAGTGGTATCAGAATGACTTTGTGGTTTCTATTGG $\begin{array}{ll}\text { AGTTGGCAGGGCTTCTCTATTCTCCTCCCGGAACAAGGGGTCTTCTGGGCTCACACCAGCAAGGATGGCAAGAGGCCACTGAA } & \text { AP-1 site } \\ \text { CCAGTCGGGGA } & \underline{\mathrm{kB} \text { site }}\end{array}$

\section{AP-1 site}

TAAAGGATGTAGCATTCCTTCTGAGTGTGTGTATCAGGCACATAGGAAAGAAAGAAATGTATTTGGGCTTTTGACCTTTTCTCAGGAGTCTCCTI GTGCAGTGGTCTCTGCTCCTCCCAGCCCCCTGTTCCTCTGAGAAGGAAGCTTCTGCTTGCTGGGCCCATTCTTCAAGGGAAGAAACGAAGACCAG AGAGTGATTTTCAAGGGTTTCCCAGAAAGTTGCAAGCTGCGTGCCTTGTTTGGCTCGCCTCTCAGATTAGCAACAACTGGTATTTCTTGCTGCTT

Enhancer I CCTGTGAGTCAGGCCTTGTAATACCTACTAATAGCTGAACCCCAGTCTTACCAATGCAGGGCCCCTGCTTAGTGTTTCATACTCAGTAGTGTGG GAACCCCTAATGCTCTGGCAGTTTGCATTCTCTATCTCACACACAGATGGGGAAGCCGACAGATAAGTCGGGACCTGCTACCCAGTAGCAGTGC TGACTTGCTCTATTCATTCATTTATTTCTGAGACACTCACCATGCGTCCTTGGCTGTCCTGGAGCTCACTATGTGACTTCCATCCTAGGCCTAAA CATAGTGTCATTGAAC GGTCTG

$$
\text { AP-1 site }
$$
ACTCTTCAGCCATCTGGTAAACCAAATGAGGGTATGGATGAGTCACCTCCACTGAGTCCCCTCTTACGGGTAATTCCCTTACCTGGCCATCTGTC ATTCACTGGACAATGGACTGCCATGGCCCTTGGATGCTCGTGACTGTGGAGAAGCACAAGGAGGTCGGGTCTTTTGAGTCCCACCAGTGGACAGT

Promoter GCTGTGTGAGTGGCCAGAGTCTCAGGGGTGGGGAGGGGAGGGAAGGGCTGGACCTGGGTCTGGAGCTCAGATGGGCTGAAACCCCAGCTTCCTGC CACCCCCATGCTTCCCCCTCTGGGAAGGGAATTACGTTTCCCCATTTTAGCCAGGGAAGACTTAGTGAACACAAAGCTGAAAGTACAAGTAGGA CAGAAAGTGAAACTGGGCGCAGCCCCCAGTATAAGACCCCCCTACCCAGGAGATGGCTGCACACAGAGGCTGGGCCCTGACATG +1

IRF site

fig. S15. Putative binding sites in the promoter region and the three upstream enhancers in the $1 / 27 p 28$ locus. 
C
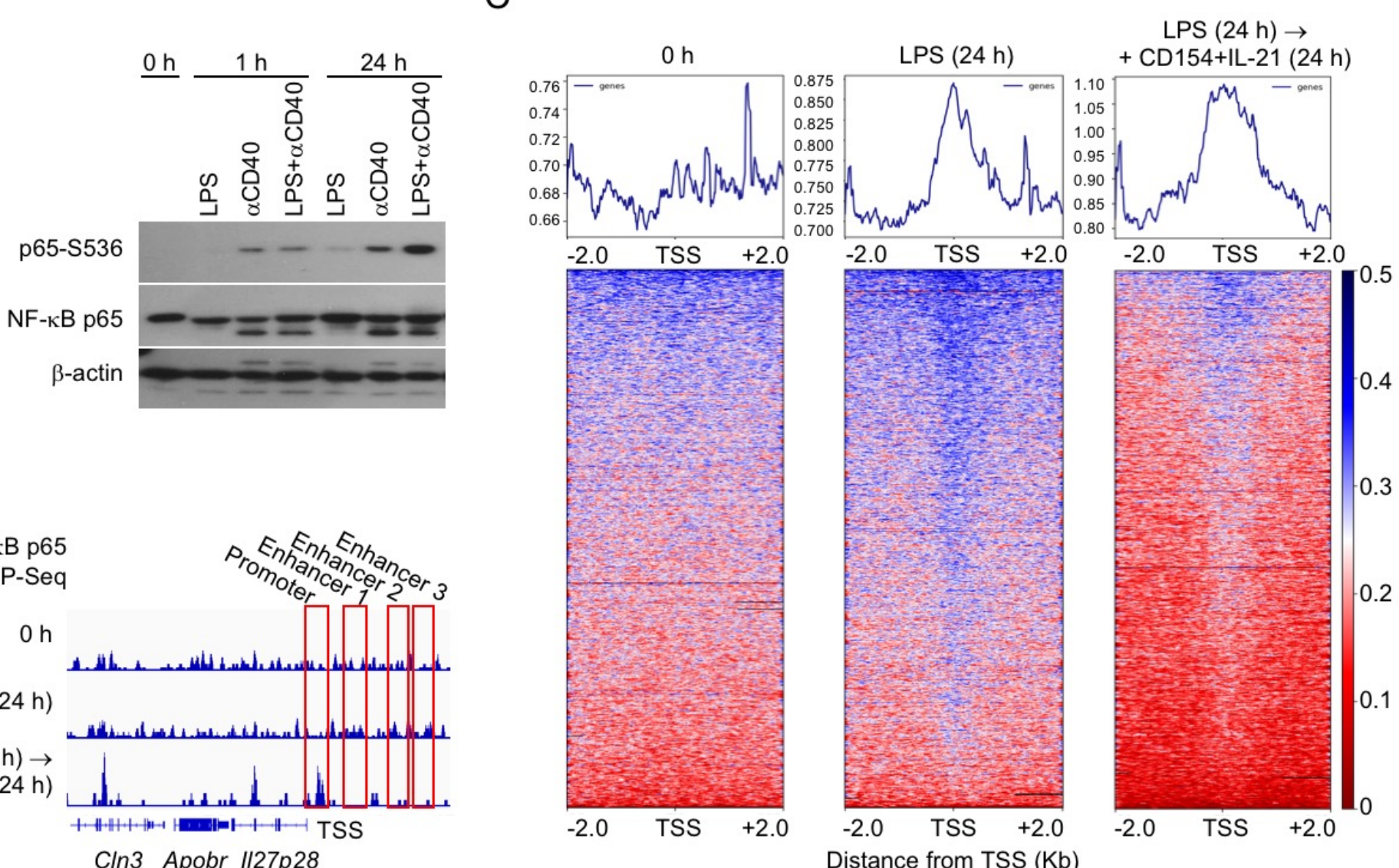

NF-kB p65 ChIP-Seq (motif enrichment) $\quad p$ value Dataset

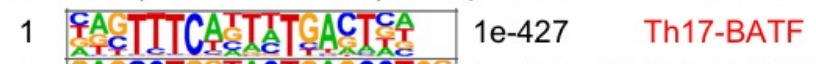
2 GACCCTCCTACTCACCCTG: 1e-127 HEK293-ZNF322 3 GGCGCIGTGTCC

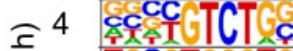
ป 5 TCCTICGCC in 6 AGATGTEAGATAAE $\neg 7$ ACIITCACIITC

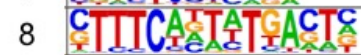
9 TECACCAA 10 GTCCCATCCC 1e-63 HEK293-KLF10 1e-37 ESC-SMAD4 K562-EGR1 iTreg-GATA3 HeLa-PRDM1 PDC-IRF8 MCF7-ARNT Promoter-NRF

B

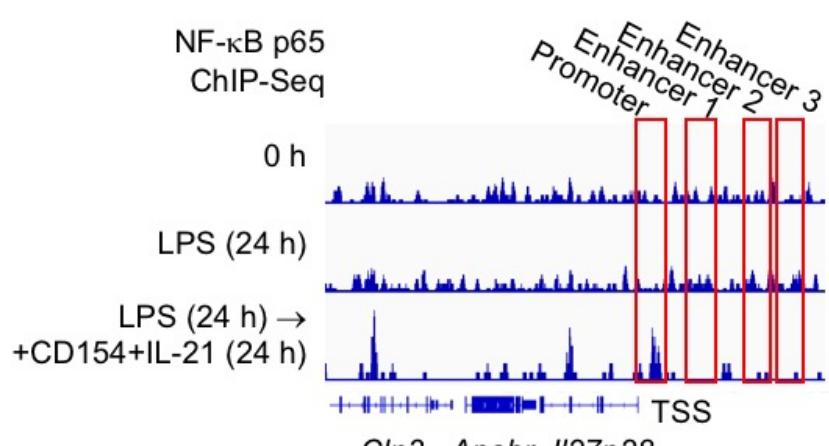

Distance from TSS $(\mathrm{Kb})$

尺े 1 GGGGGTGTGTCC 10 -1328 HEK293-KLF10

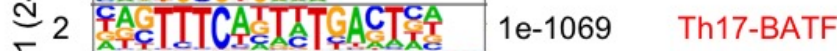
N 3 GACCTCETACTEACCTGS 1e-209 HEK293-ZNF322 F 4 ACTITCATITCS $1 \mathrm{e}-72$ HeLa-PRDM1

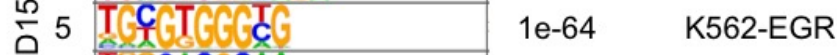
06 TECACCCAA $1 \mathrm{e}-42$ MCF7-ARNT $\uparrow 7$ CTIICA I 8 CTCCCATCCC 1e-17 MCF7-NRF1 ป 9 CIAATICAATTA $1 \mathrm{e}-17$ GHFT1-PROP1

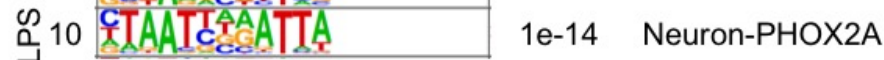

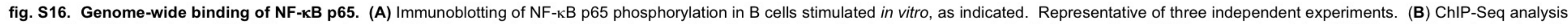

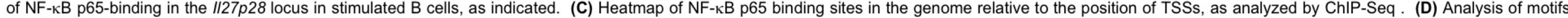
enriched in NF-kB p65 binding sites. 
A

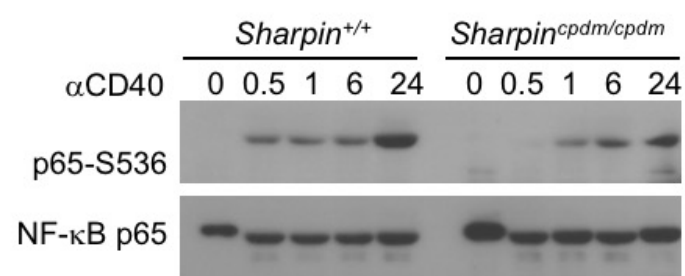

B

LPS+CD154+IL-21

Ebi3 expression

to $\mathrm{Cd79b)}$

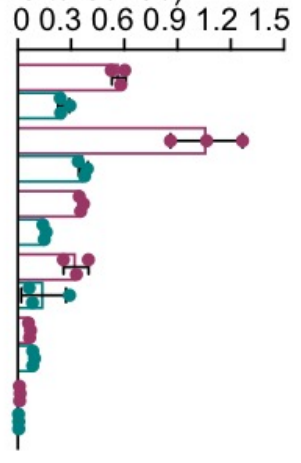

C

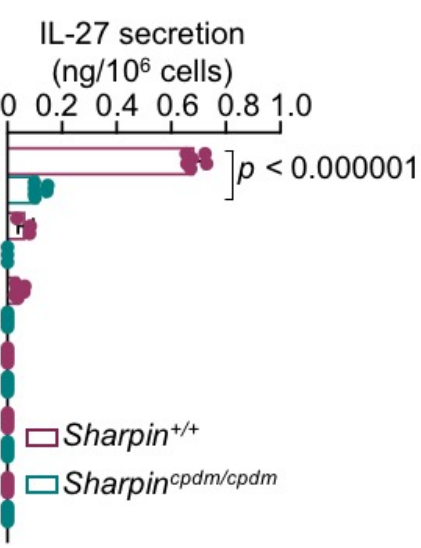

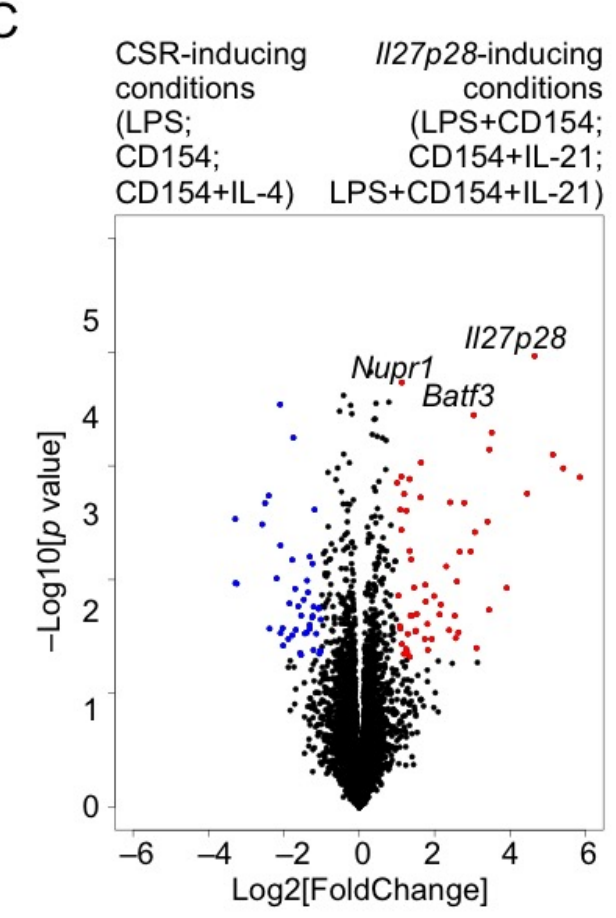

fig. S17. SHARPIN promotes NF-kB p65 activation and IL-27 induction. (A) Immunoblotting of phosphorylation of NF-kB p65 at Ser355 (p65-S355) in B cells stimulated in vitro, as indicated. Representative of two independent experiments. (B) qRT-PCR analysis of II27p28 and Ebi3 expression in Sharpin ${ }^{+/}$and Sharpin ${ }^{\text {cpdm/cpdm }}$ B cells stimulated for $48 \mathrm{~h}$ (left and middle) and ELISA of IL-27 secretion by these cells (right). (C) Volcano plot depicting DEGs in B cells stimulated with I/27p28-inducing conditions and those stimulated with CSR-inducing conditions, as indicated, for $48 \mathrm{~h}$ 

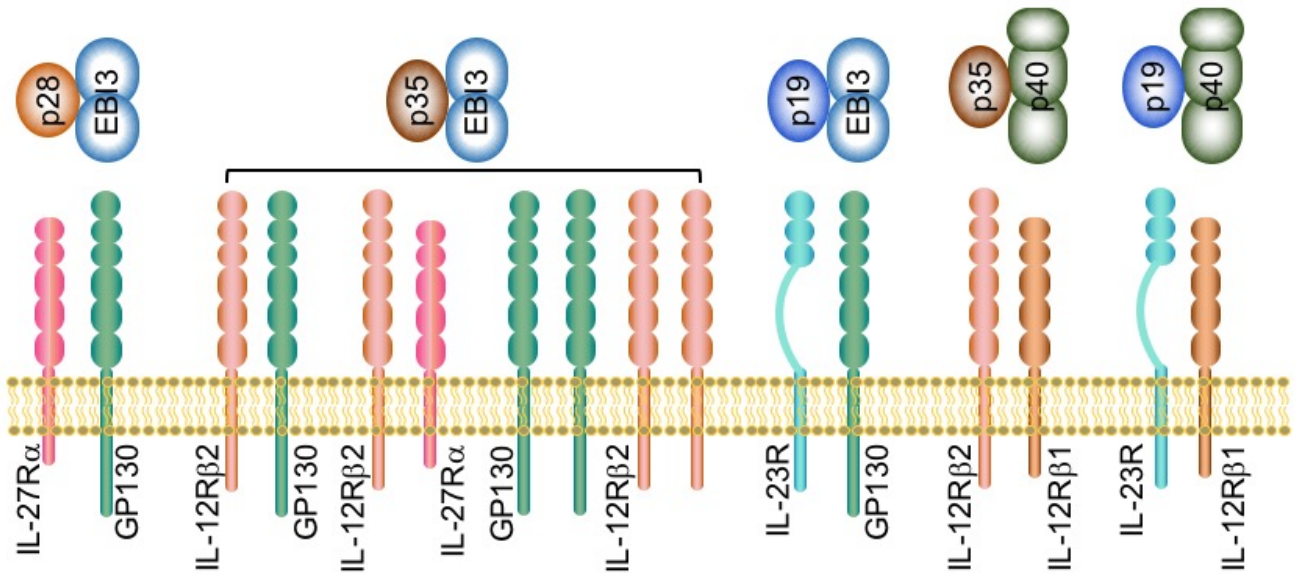

IL-6 family
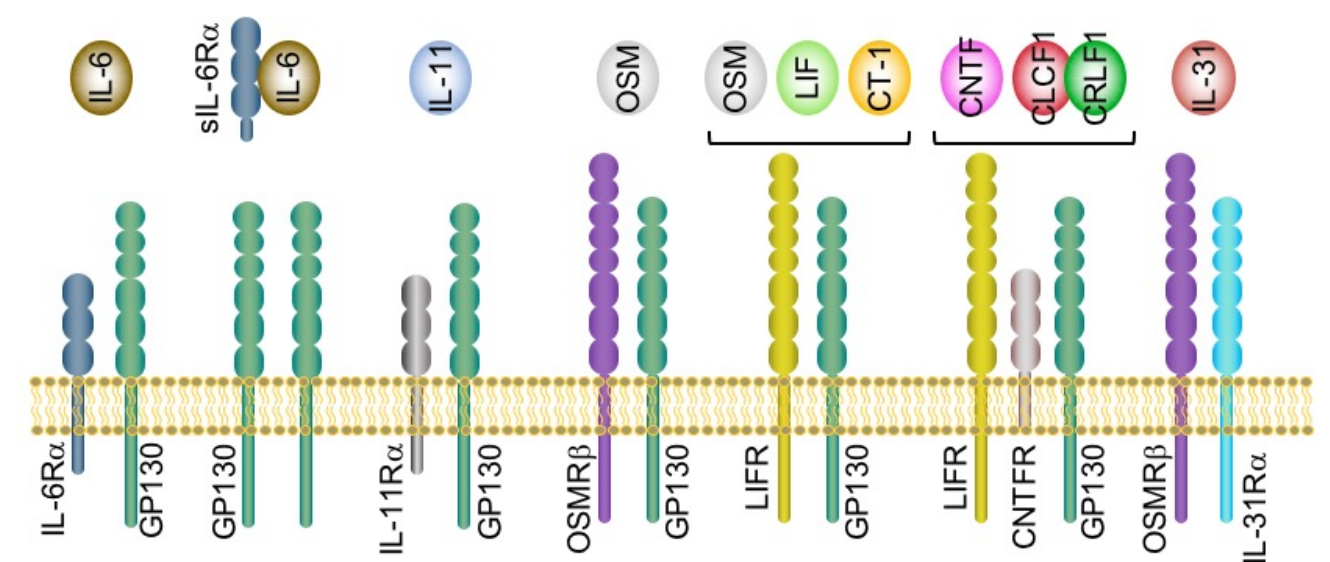

D
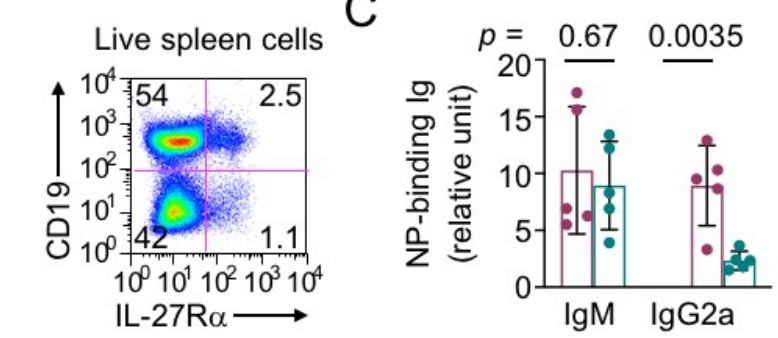

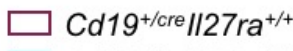
$\square \mathrm{Cd}^{\mathrm{a}} \mathrm{9}^{+/ \mathrm{cre}} / 127 \mathrm{ra} \mathrm{flft}^{\mathrm{f}}$ NP-CGG/alum/LPS (d 14)

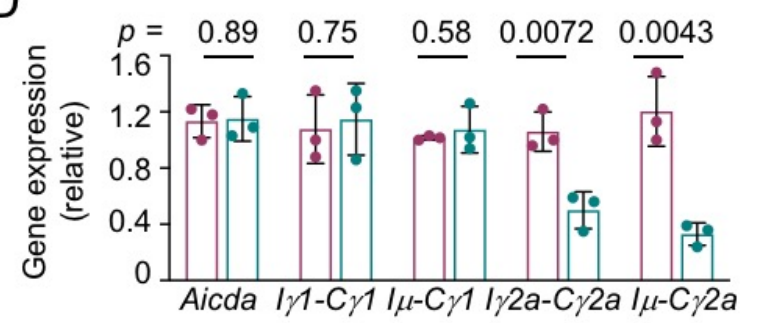

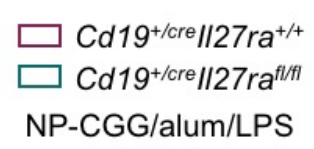
NP-CGG/alum
(d 14)

E

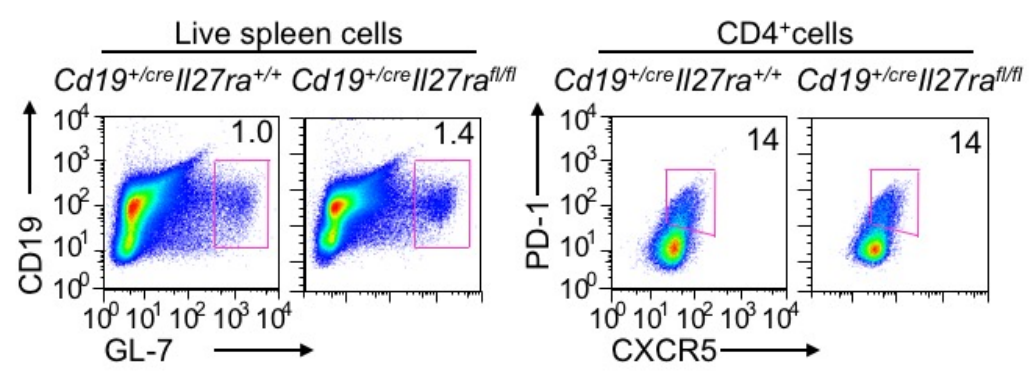

fig. S18. B cell-intrinsic role of IL-27 receptor signaling in IgG2a response in vivo. (A) Illustration of cytokines in the IL-12 family, IL-6 family and related forms as well as their respective receptors. (B) Flow cytometry analysis of IL-27R $\alpha$ in spleen cells in non-immunized C57 mice. Representative of three independent experiments. (C-E) ELISA of NPbinding $\operatorname{lgM}$ and $\lg$ G2a (C), qRT-PCR analysis of gene expression (D), and flow cytometry analysis of $B$ cells and $T$ cells $(E$; representative of three

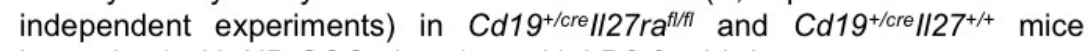
immunized with NP-CGG plus alum with LPS for $14 \mathrm{~d}$. 


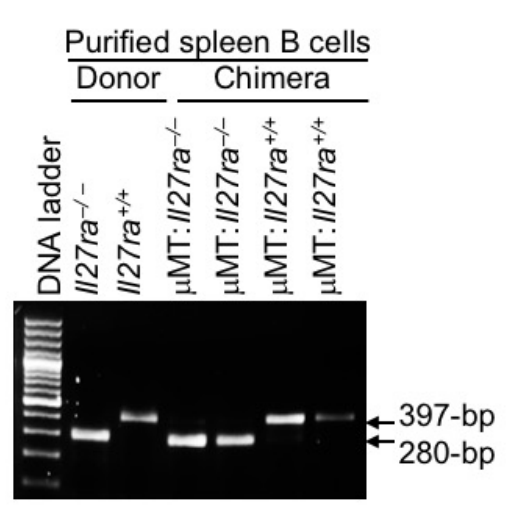

C
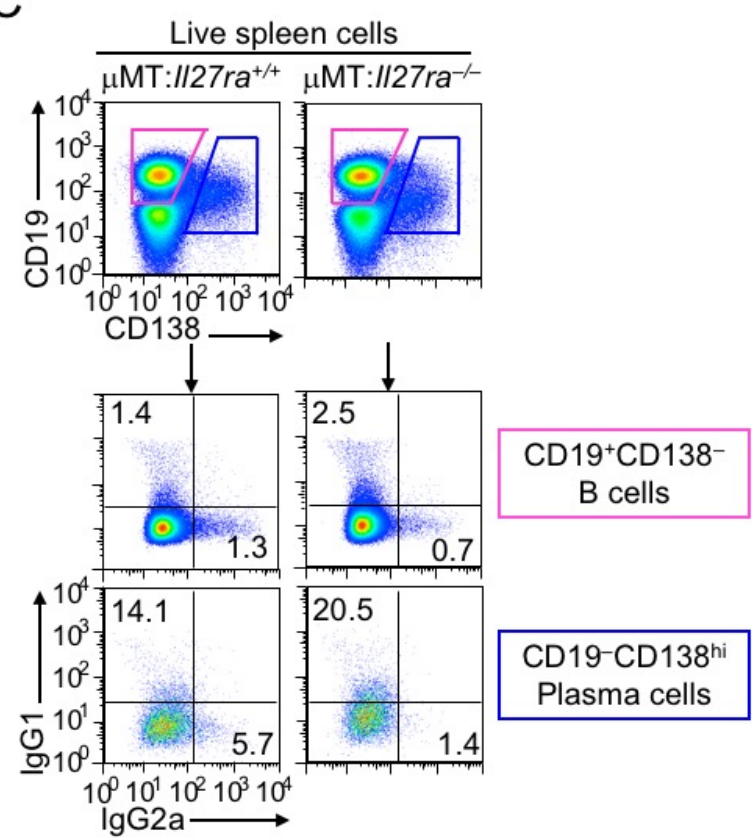

$\mathrm{NP}_{4}$-binding ASCs in the bone marrow $\lg \mathrm{M}^{+}$

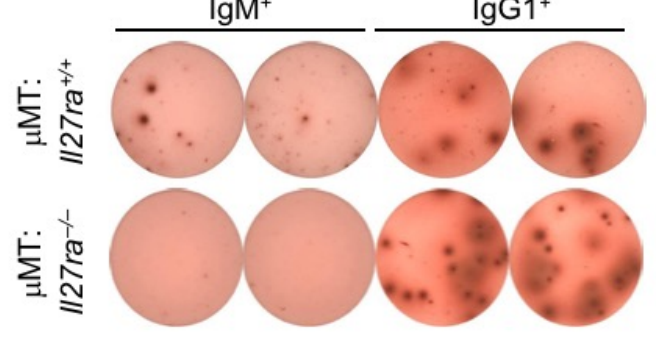

+NP-CGG/alum/LPS (d 14)

D

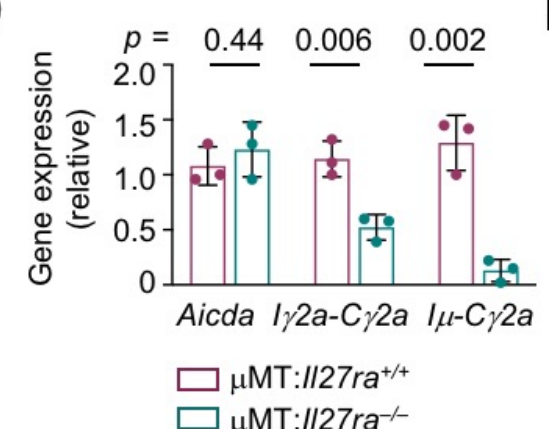

E

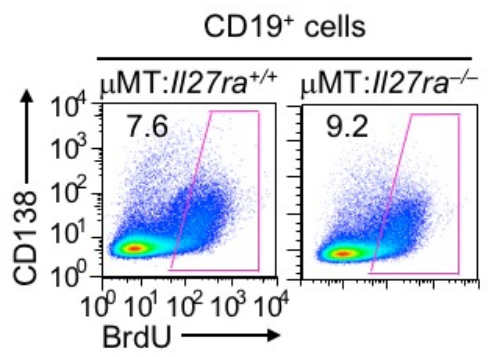

$\mathrm{F}$

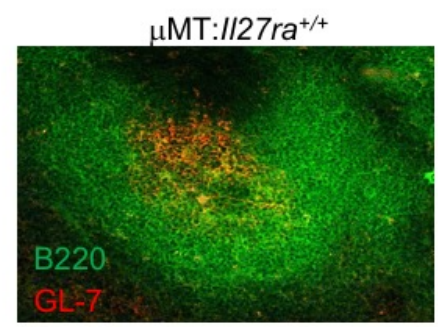

$\mathrm{NP}_{4}$-binding ASCs in the spleen $\lg \mathrm{M}$

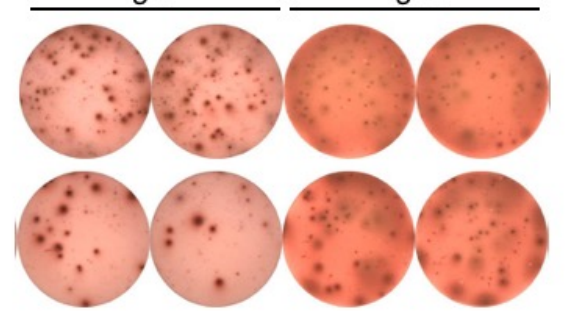

$\mu \mathrm{MT}: / / 27 \mathrm{ra}^{-/-}$

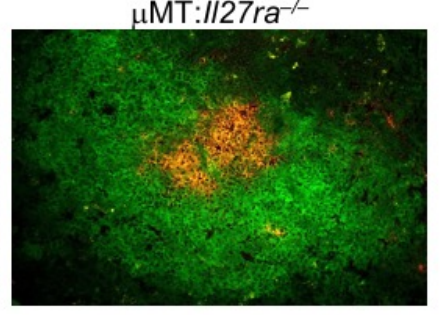
/LPS (d 14)

$p=0.000004 \quad \underline{0.0004} \quad 0.00015 \quad \underline{0.13}$

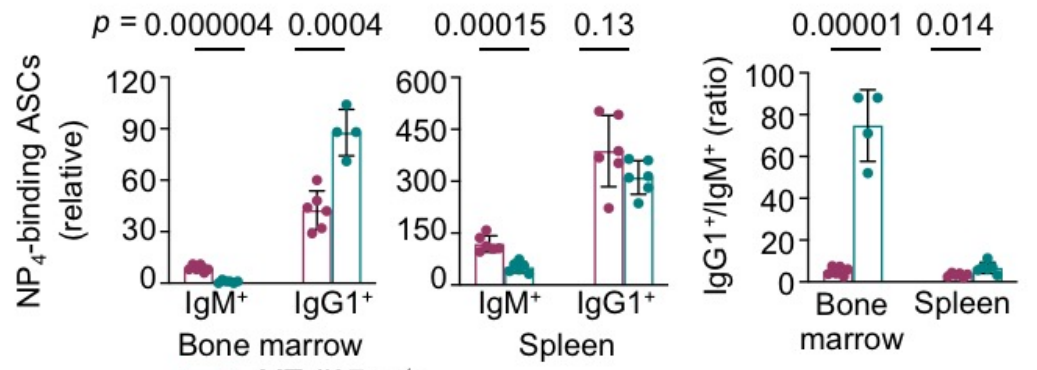

Bone marrow

Spleen

marrow

$\square \mu \mathrm{MT}: / 127 \mathrm{ra}^{-/}$

G
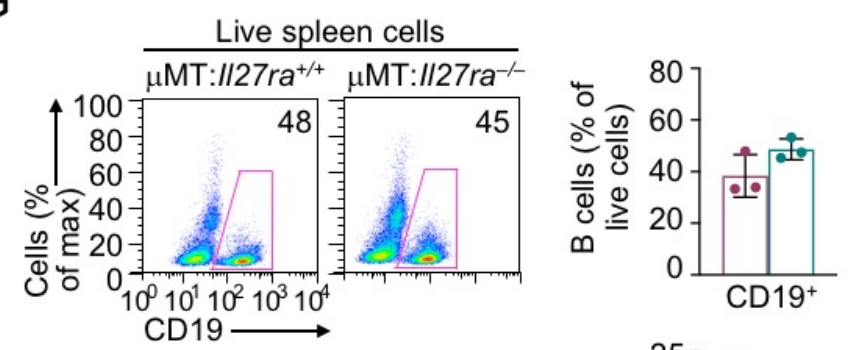

NP-CGG/alum

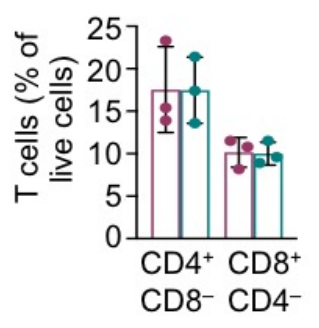

$\square \mu \mathrm{MT}: / / 27 \mathrm{ra}^{+/+}$ +NP-CGG/alum/LPS (d 14) $\square \mu \mathrm{MT}: / / 27 \mathrm{ra}^{-/}$

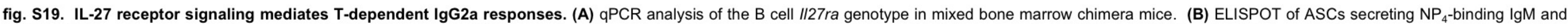

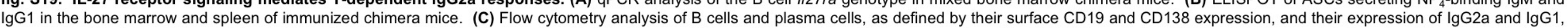

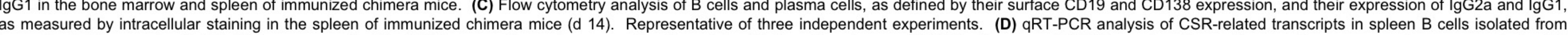

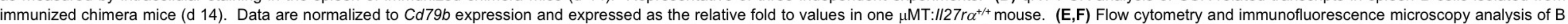

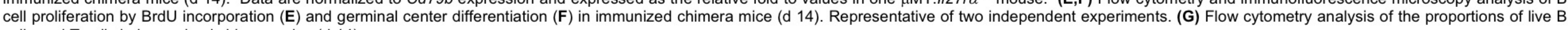
cells and T cells in immunized chimera mice (d 14). 
A
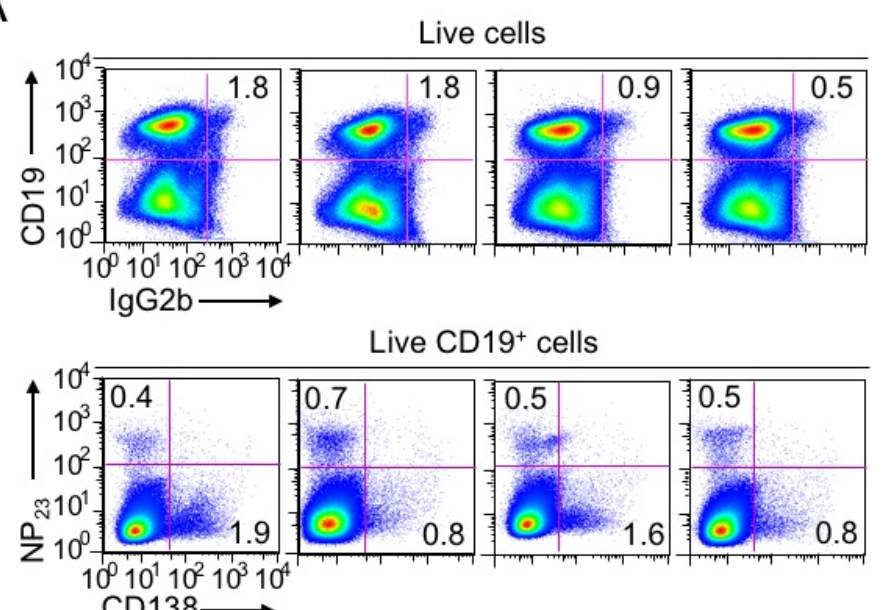

CD138 $\longrightarrow \quad$ Live cells

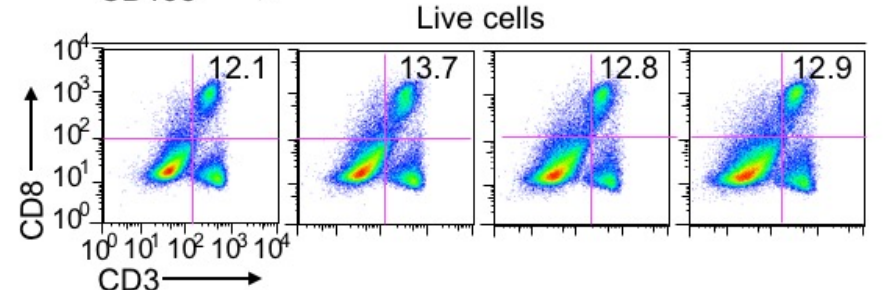
$\mathrm{CD} 3 \longrightarrow$
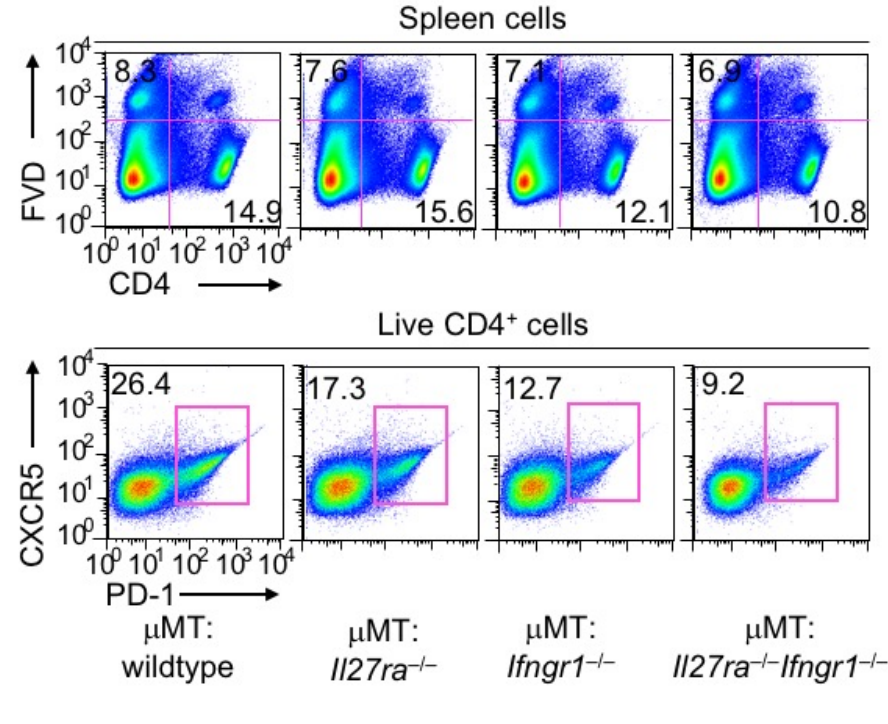

(+ NP-CGG/alum/LPS, d 14)
B

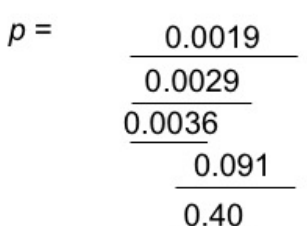

$\frac{0.0079}{\frac{0.40}{0.25}} \frac{0.054}{\underline{0.29}}$

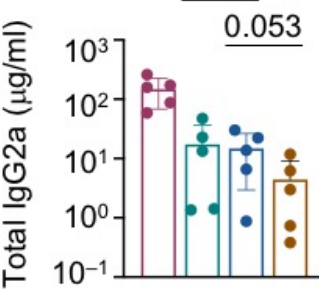

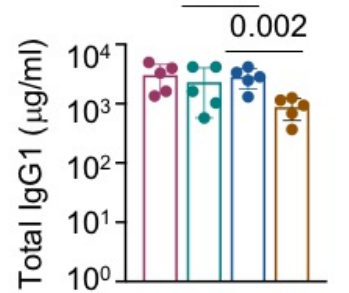

$\square$ Wildtype

$\square / 127 \mathrm{ra}^{-1-}$

$\square / 27 \mathrm{ra}^{-1-l}$ fngri-1-

C

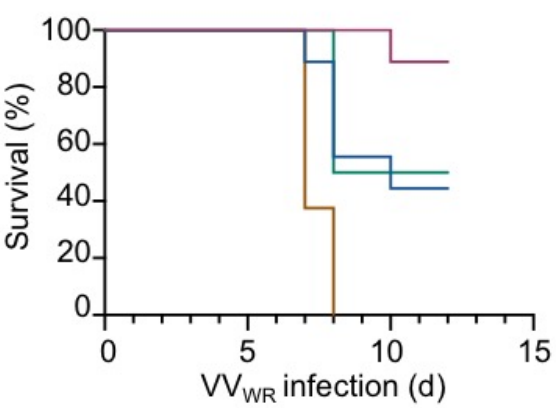

- $1127 \mathrm{ra}^{-1-}(\mathrm{n}=8)$

- Ifngr1 ${ }^{-1-}(\mathrm{n}=9)$

- $127 \mathrm{ra}^{-1-}$ Ifngrt ${ }^{-1-}(\mathrm{n}=8)$

$p=$

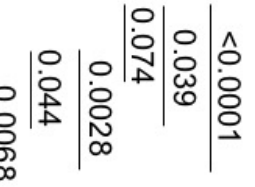

D

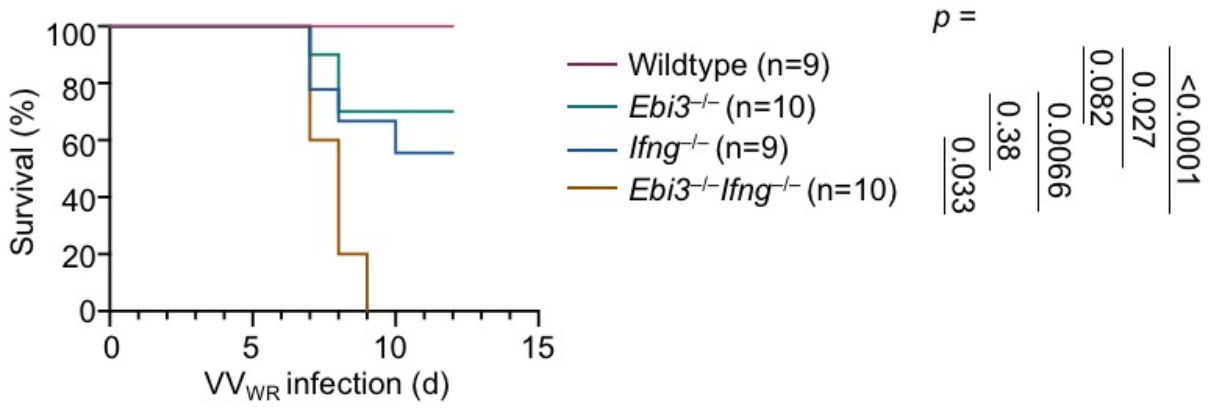

fig. S20. Synergy of IL-27 receptor and IFN $\gamma$ receptor signals in vivo. (A) Flow cytometry analysis of $\mathrm{B}$ cells and T cells in double and single KO chimera mice, as indicated, after immunization. Representative of five mice in each group. (B) ELISA of total $\lg \mathrm{G} 2 \mathrm{a}$ and $\operatorname{lgG} 1$ in non-immunized mice with double and single $\mathrm{KO}$ in cytokine receptor genes, as indicated. (C,D) Survival analysis of mice with double and single $\mathrm{KO}$ in cytokine receptor genes (C) or cytokine genes (D), as indicated after infection with $V V$. Mice that had lost more than $25 \%$ of the initial body weight after infection with $\mathrm{V}_{\mathrm{WR}}$. Mice that had lost more than $25 \%$ of the initial body weight were considered moribund and sacrificed. 
A

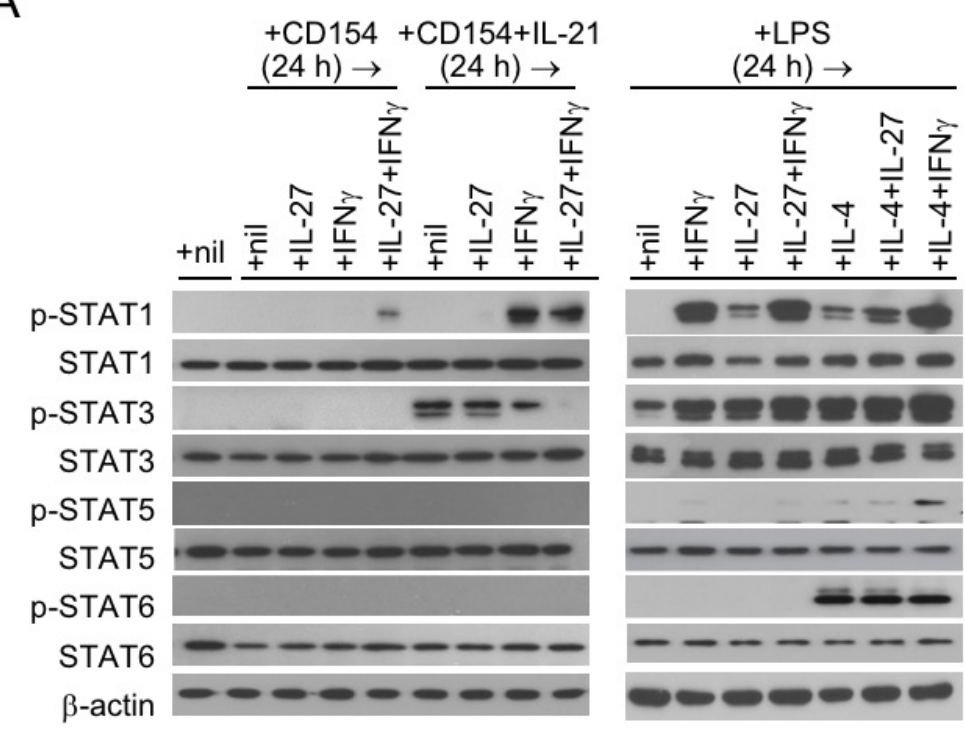

C

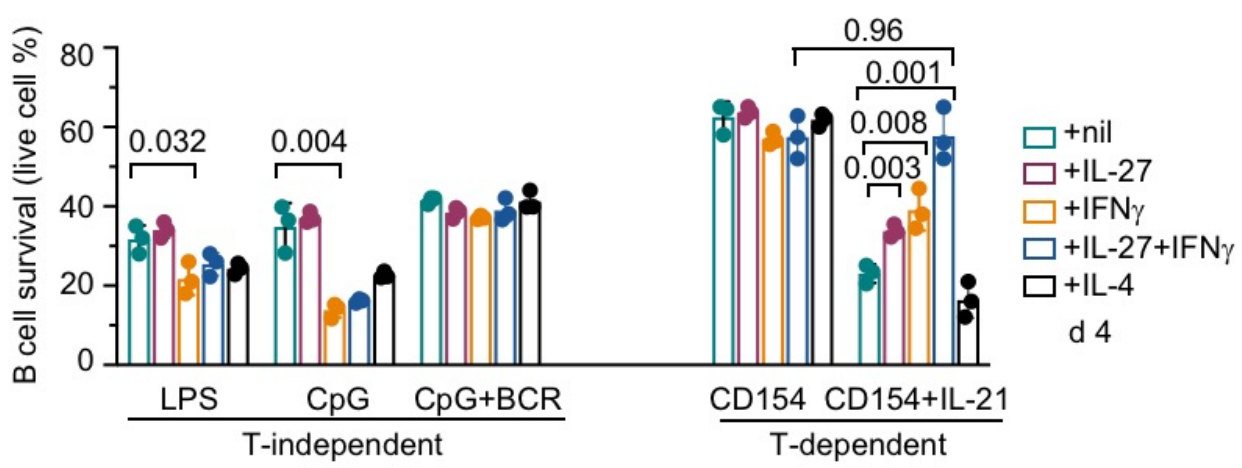

B

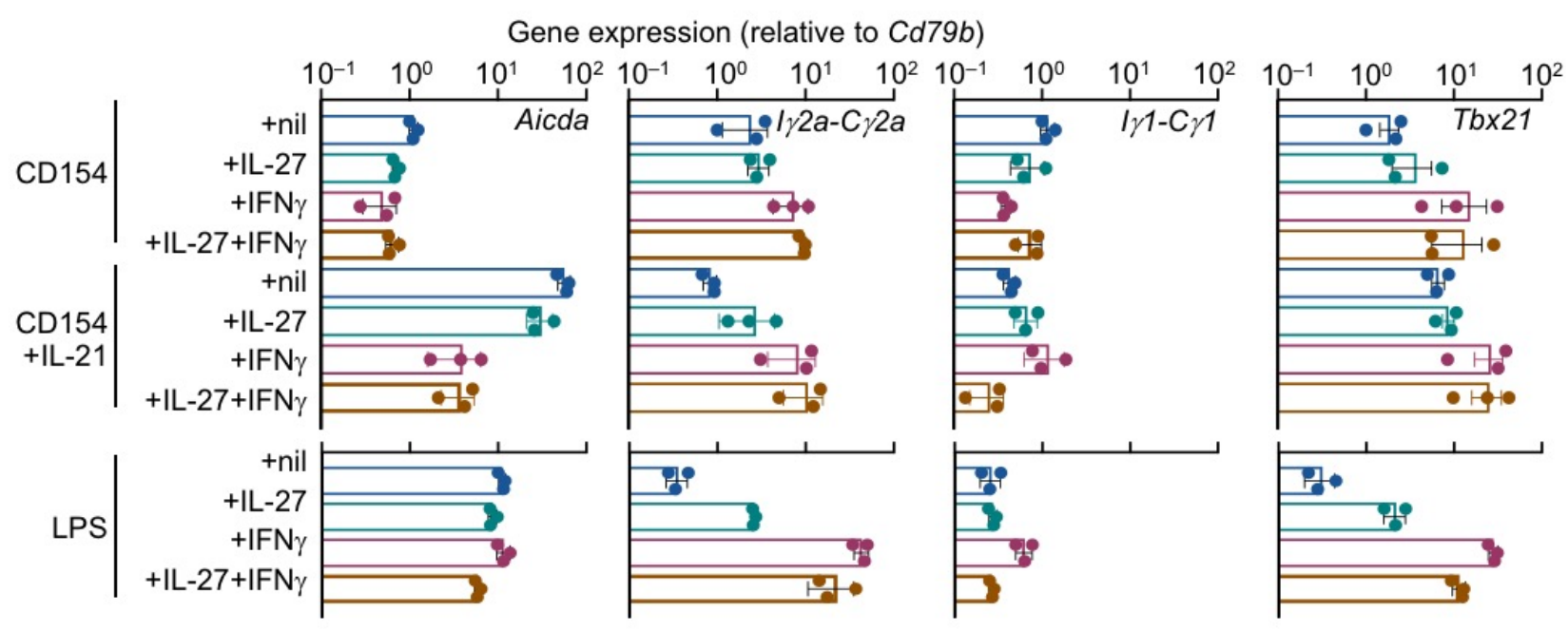

D

E

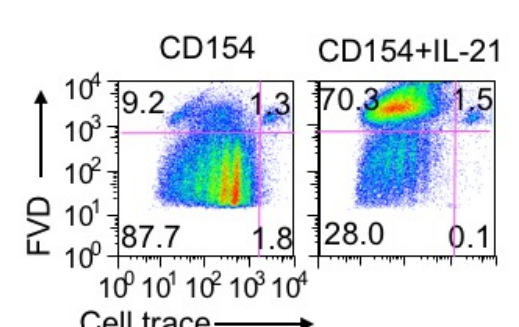

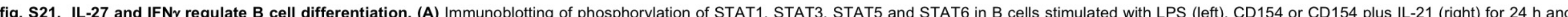

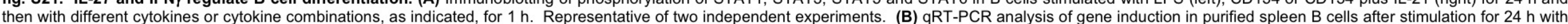

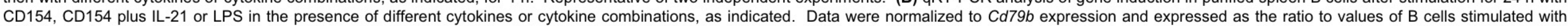

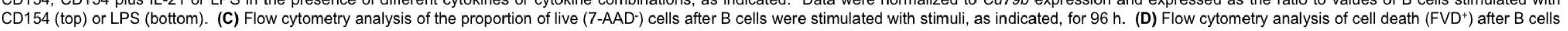
were stimulated with CD154 plus IL-21, as indicated, for $96 \mathrm{~h}$. (E) ELISA of IgM and IgG2a in B cells cultured for $96 \mathrm{~h}$ in the presence of LPS plus nil, IL-27 or IFN $\gamma$, as indicated. 
A LPS+IL-4 LPS+IL-4+IL-27

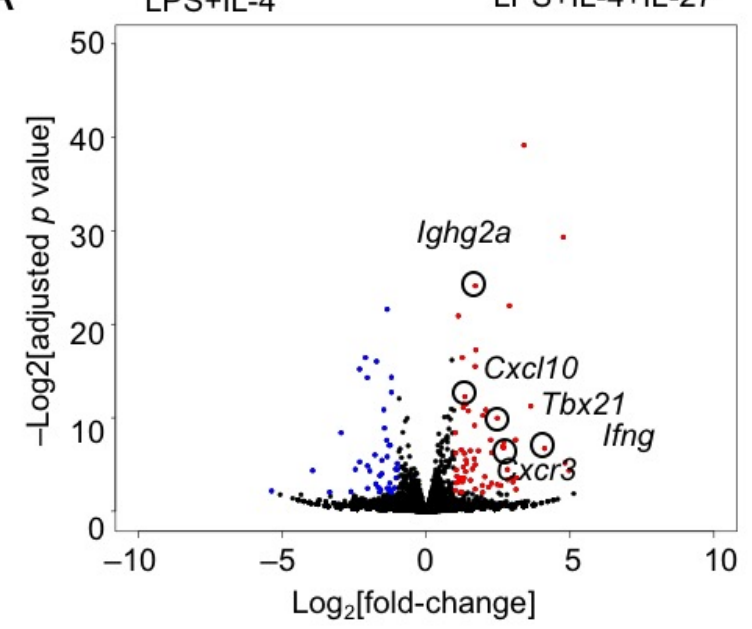

D

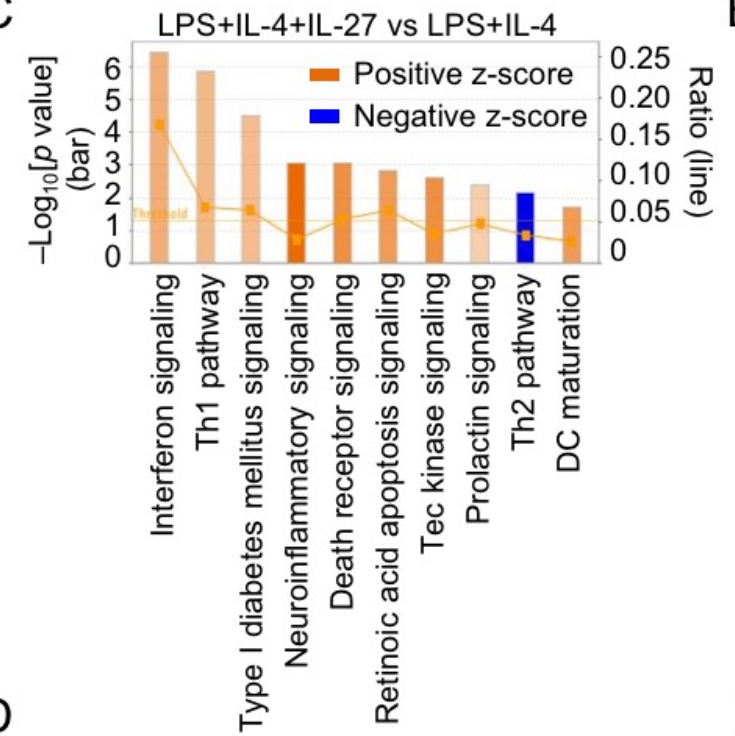

E
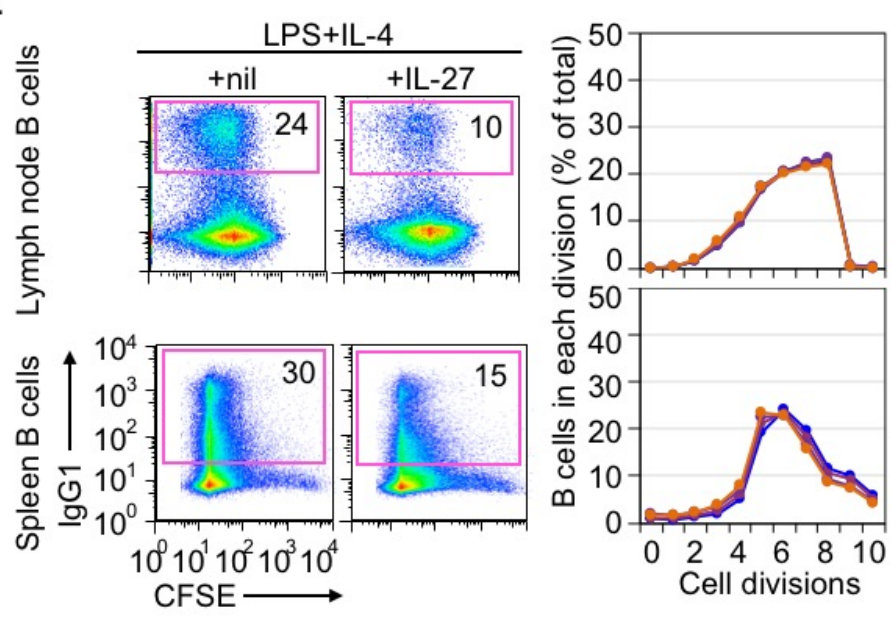

+ IL-27

$(\mathrm{ng} / \mathrm{ml})$

Average

Average

6.08

6.13

6.07

6.14

6.03

6.45

6.29

6.01

5.94
F

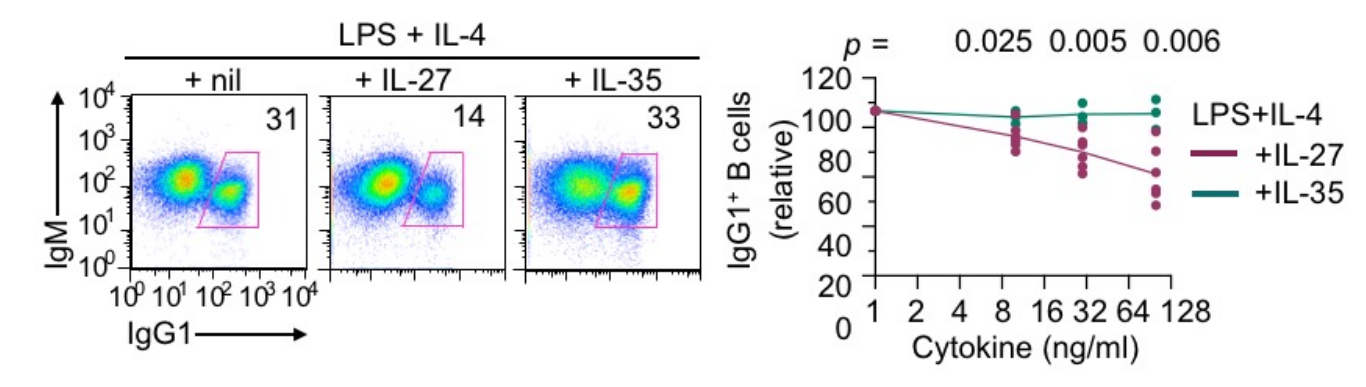

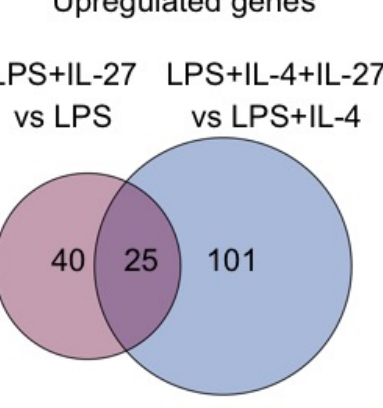

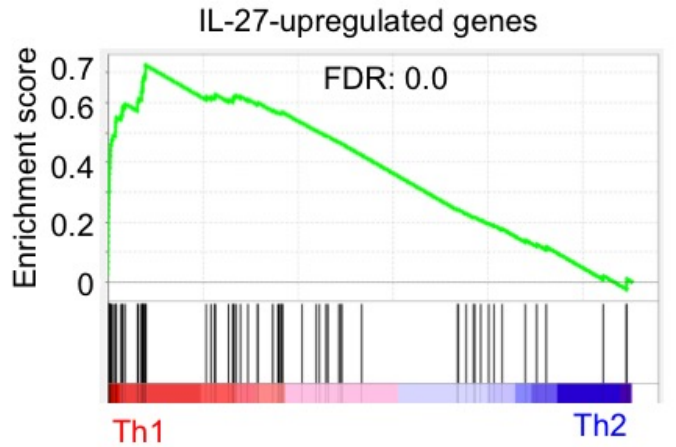

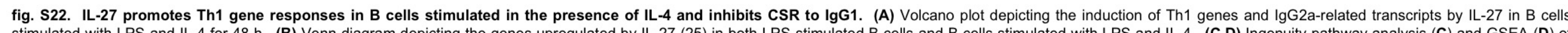

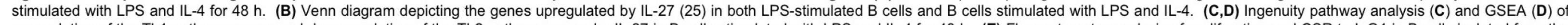

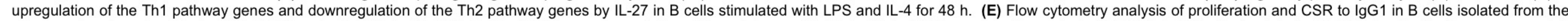

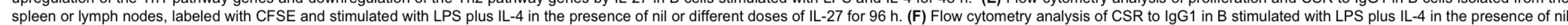
spleen or lymph nodes, labeled with CFSE and stimulated with LPS plus IL-4 in the presence
or different doses of IL-27 or IL-35 for $96 \mathrm{~h}$. Representative of two independent experiments. 
LPS $(\mu \mathrm{g} / \mathrm{ml})$

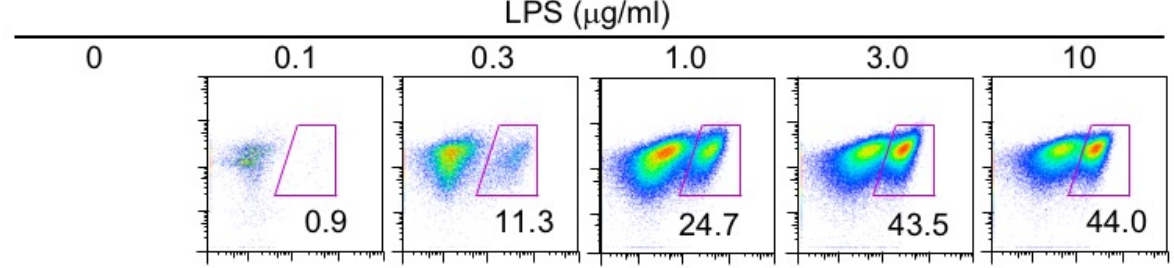

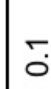
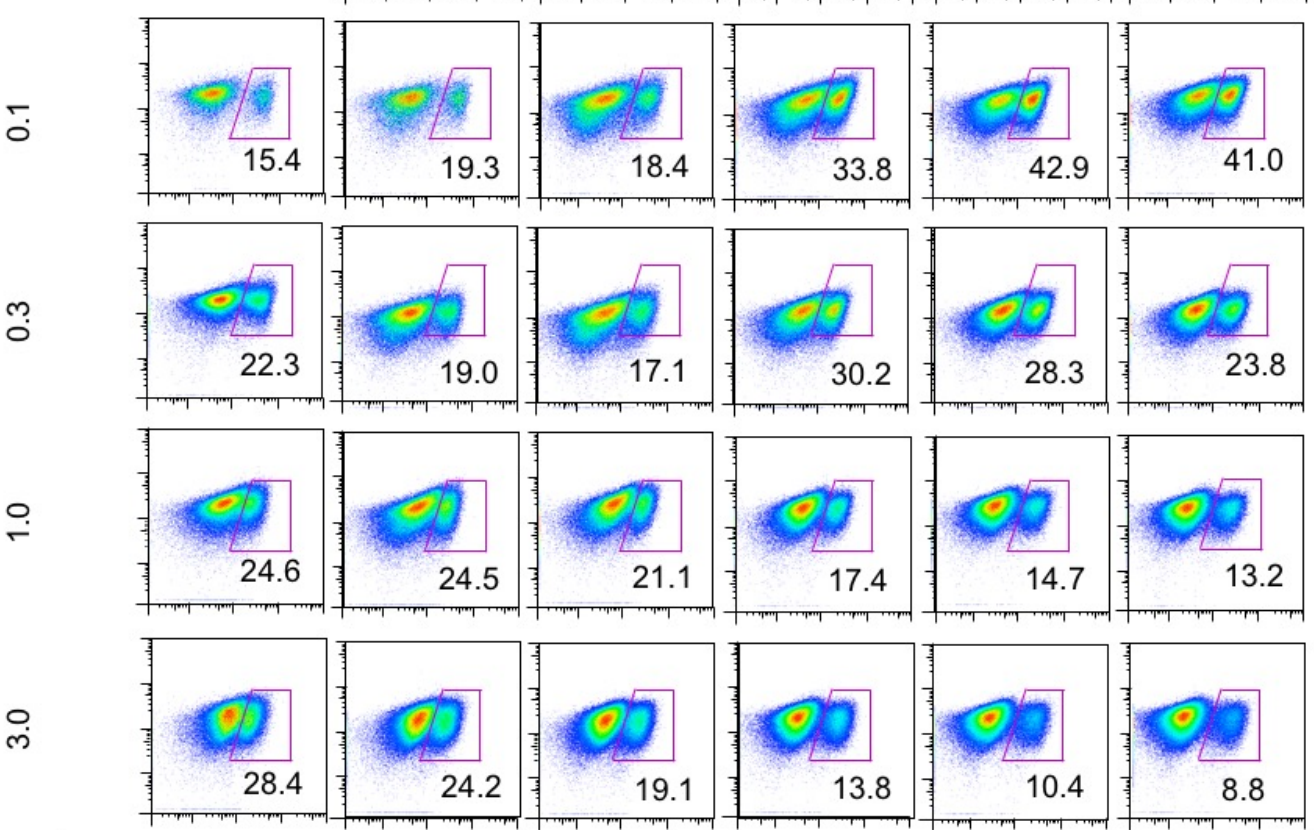

은 \begin{tabular}{l}
$10^{4}$ \\
$10^{3}$ \\
\hline \\
0
\end{tabular}

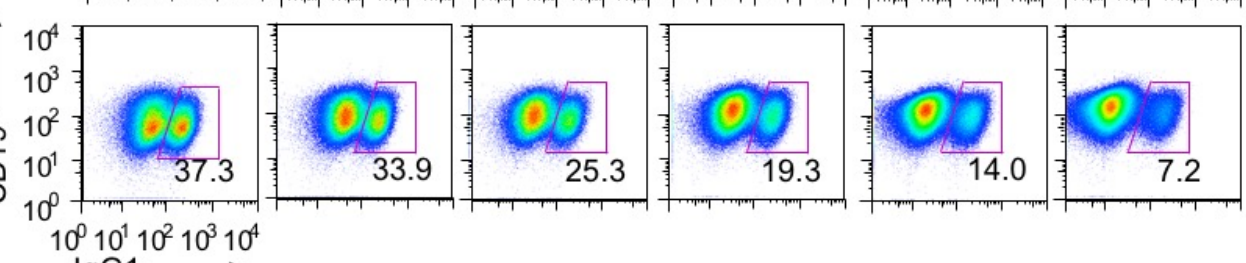

$\operatorname{lgG} 1 \stackrel{10^{2} 10^{3} 10^{4}}{\longrightarrow}$

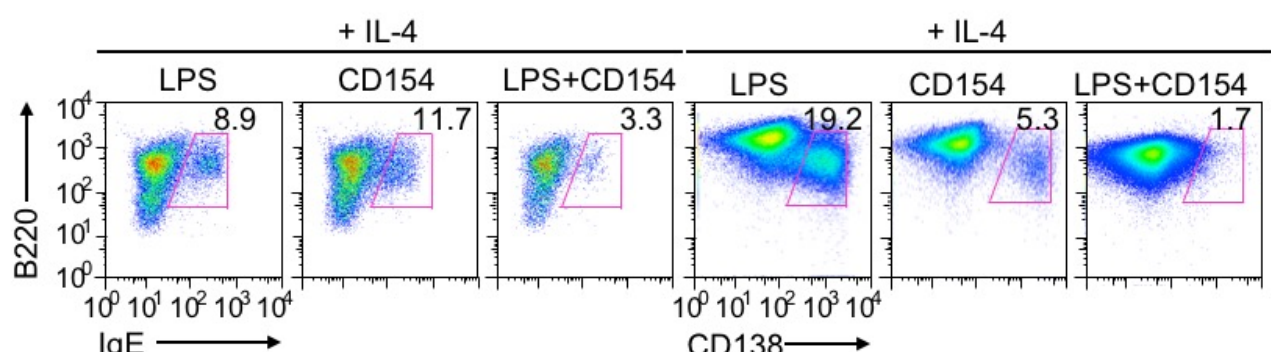

D
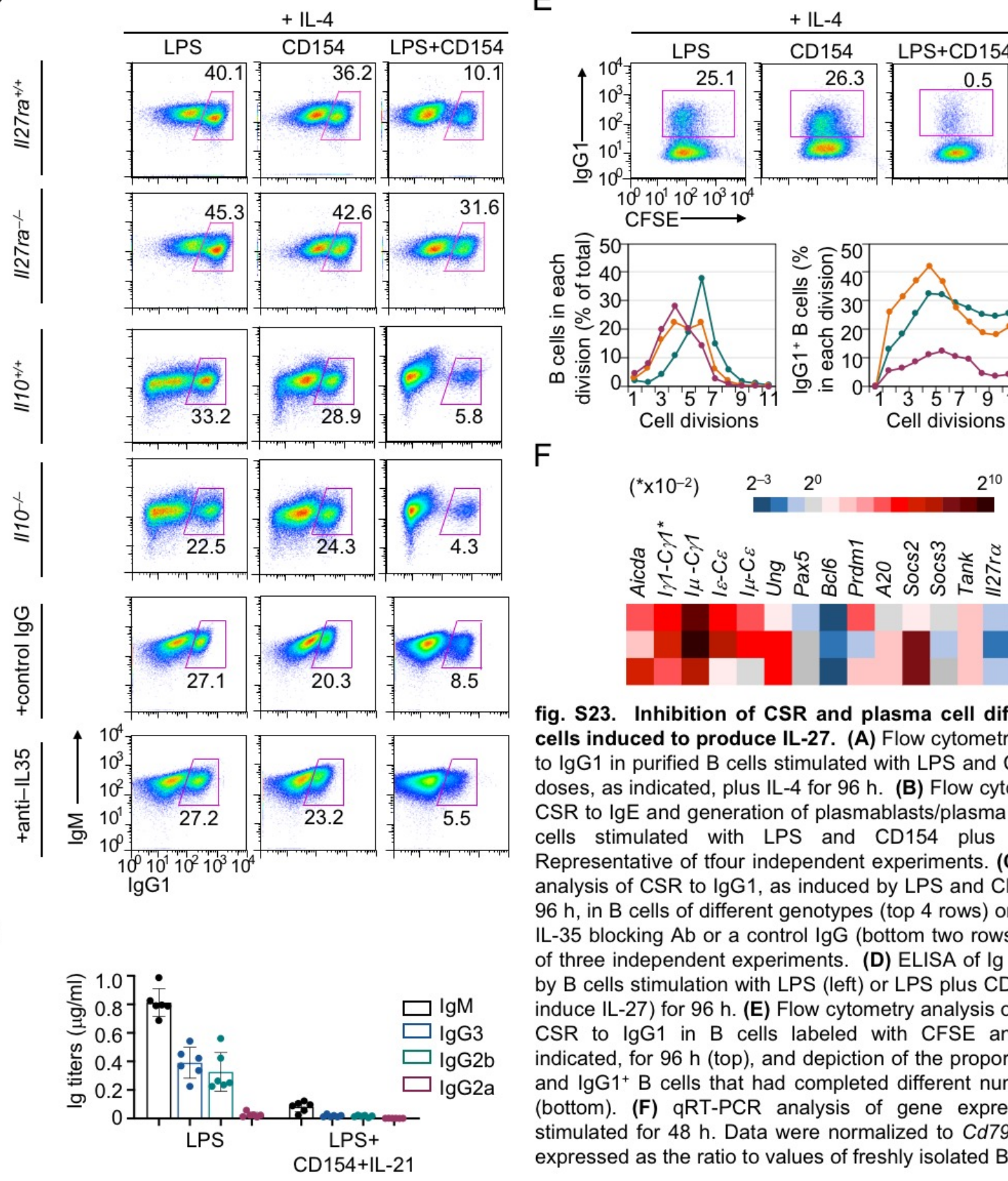

索

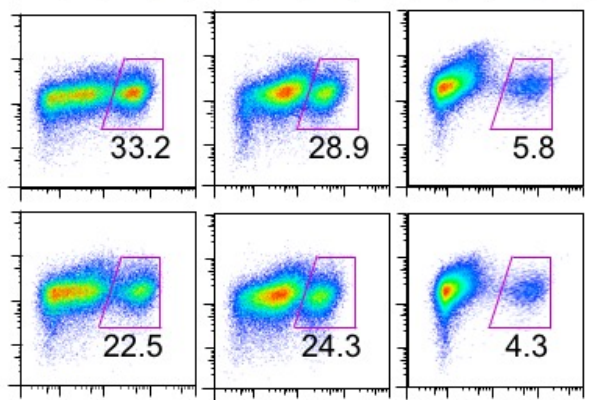

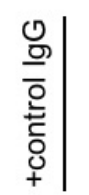
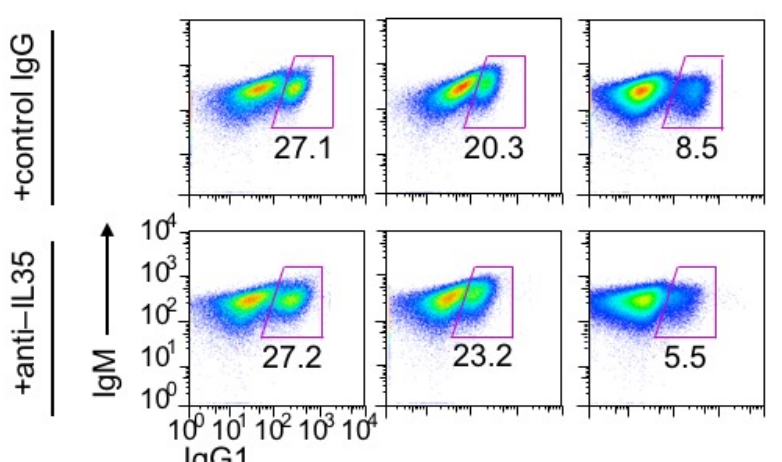

fig. S23. Inhibition of CSR and plasma cell differentiation in $B$ cells induced to produce IL-27. (A) Flow cytometry analysis of CSR to IgG1 in purified B cells stimulated with LPS and CD154 at different doses, as indicated, plus IL-4 for $96 \mathrm{~h}$. (B) Flow cytometry analysis of CSR to IgE and generation of plasmablasts/plasma cells in purified B cells stimulated with LPS and CD154 plus IL-4 for $96 \mathrm{~h}$ Representative of tfour independent experiments. (C) Flow cytometry analysis of CSR to IgG1, as induced by LPS and CD154 plus IL-4 for $96 \mathrm{~h}$, in B cells of different genotypes (top 4 rows) or treated with antiIL-35 blocking Ab or a control IgG (bottom two rows). Representative of three independent experiments. (D) ELISA of Ig isotypes secreted by B cells stimulation with LPS (left) or LPS plus CD154 and IL-21 (to induce IL-27) for $96 \mathrm{~h}$. (E) Flow cytometry analysis of proliferation and CSR to IgG1 in B cells labeled with CFSE and stimulated, as indicated, for $96 \mathrm{~h}$ (top), and depiction of the proportion of live $\mathrm{B}$ cells and $1 \mathrm{G}^{+} \mathrm{B}$ cells that had completed different numbers of divisions (bottom). (F) GRT PCR ansis of (bottom). (F) $\mathrm{CRT}-\mathrm{PCR}$ analysis of gene expression in $\mathrm{B}$ cells stimulated for $48 \mathrm{~h}$. Data were normalized to Ca79b expression and expressed as the ratio to values of freshly isolated B cells $(0 \mathrm{~h})$.

fig. S23 
A

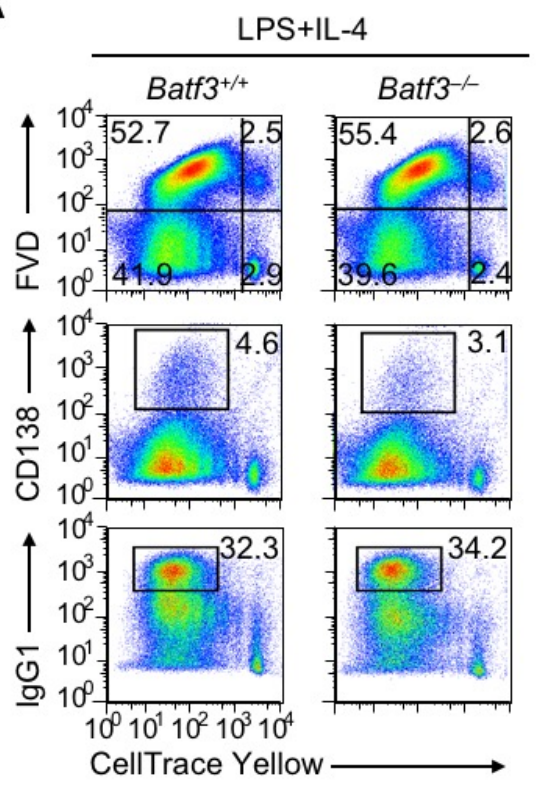

LPS
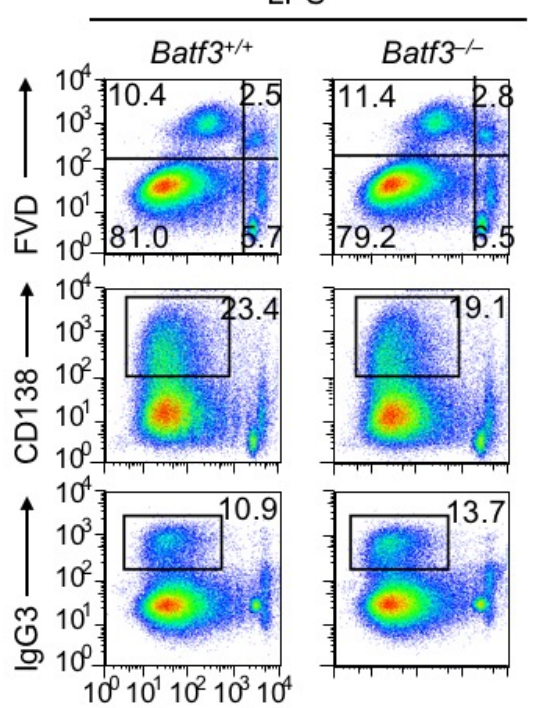

$10^{\circ} 10^{1} 10^{2} 10^{3} 10^{4}$

CellTrace Yellow
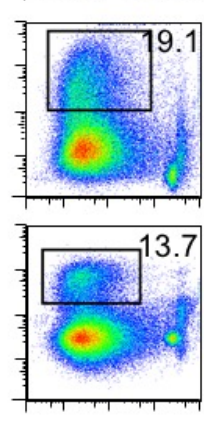

LPS+ IFN $\gamma$

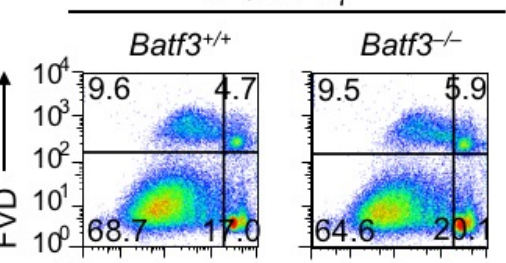

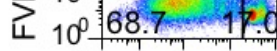
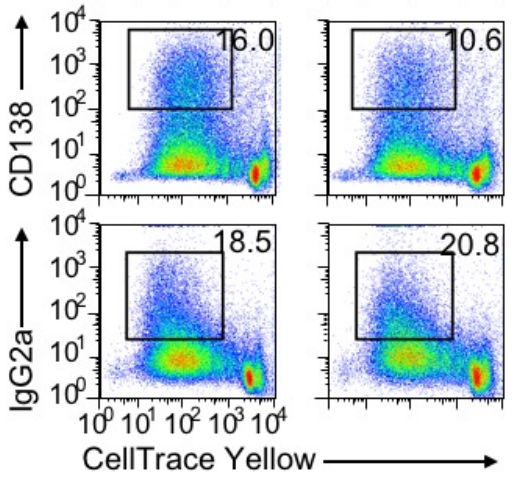

B
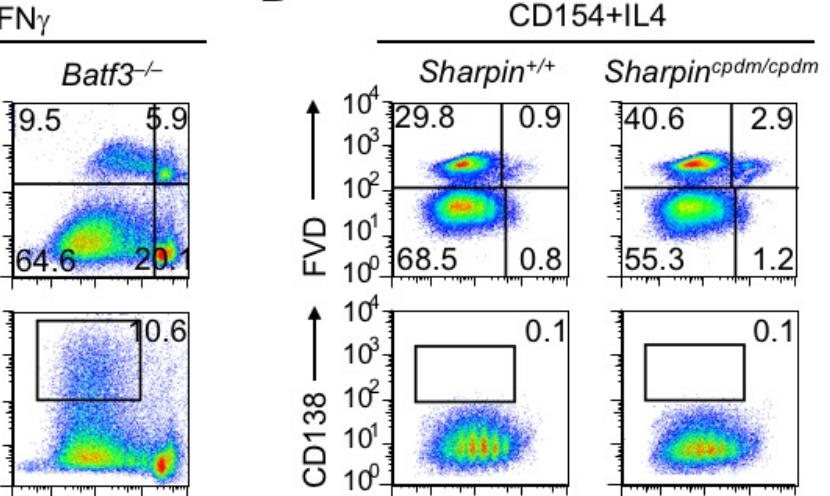

100

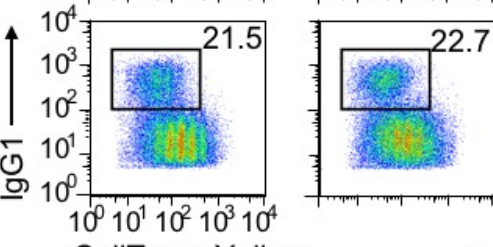

CellTrace Yellow

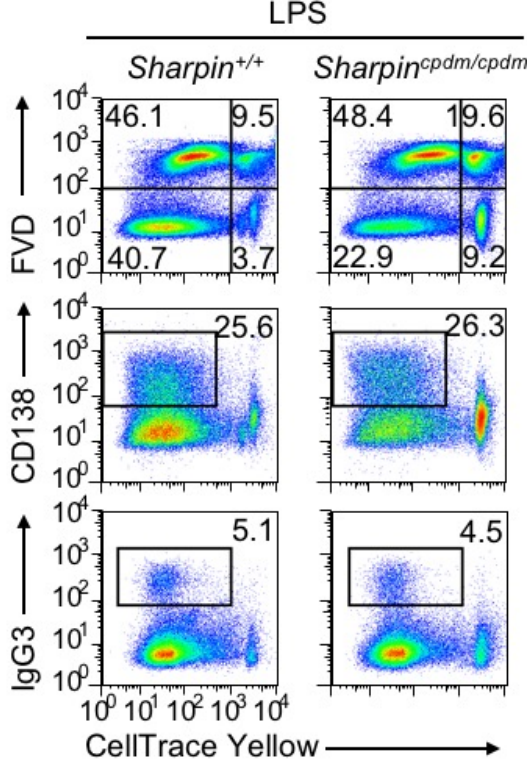

fig. S24. Normal proliferation, survival, CSR and plasma cell differentiation in B cells lacking Batf3 or expressing Sharpin ${ }^{\text {cpdm/cpdm }}$. (A) Flow cytometry analysis of proliferation, survival, CSR to appropriate IgG isotypes and plasma cell differentiation in Batf3- ${ }^{-/}$and Batf $3^{+/+}$B cells labeled with CellTrace Yellow and stimulated, as indicated, for $96 \mathrm{~h}$. Representative of three independent experiments. (A) Flow cytometry analysis of proliferation, survival, CSR to appropriate IgG isotypes and plasma cell differentiation in Sharpin ${ }^{\text {cpdm/cpdm }}$ and Sharpin ${ }^{+/+}$B cells labeled with CellTrace Yellow and stimulated, as indicated, for $96 \mathrm{~h}$. Representative of four independent experiments. 


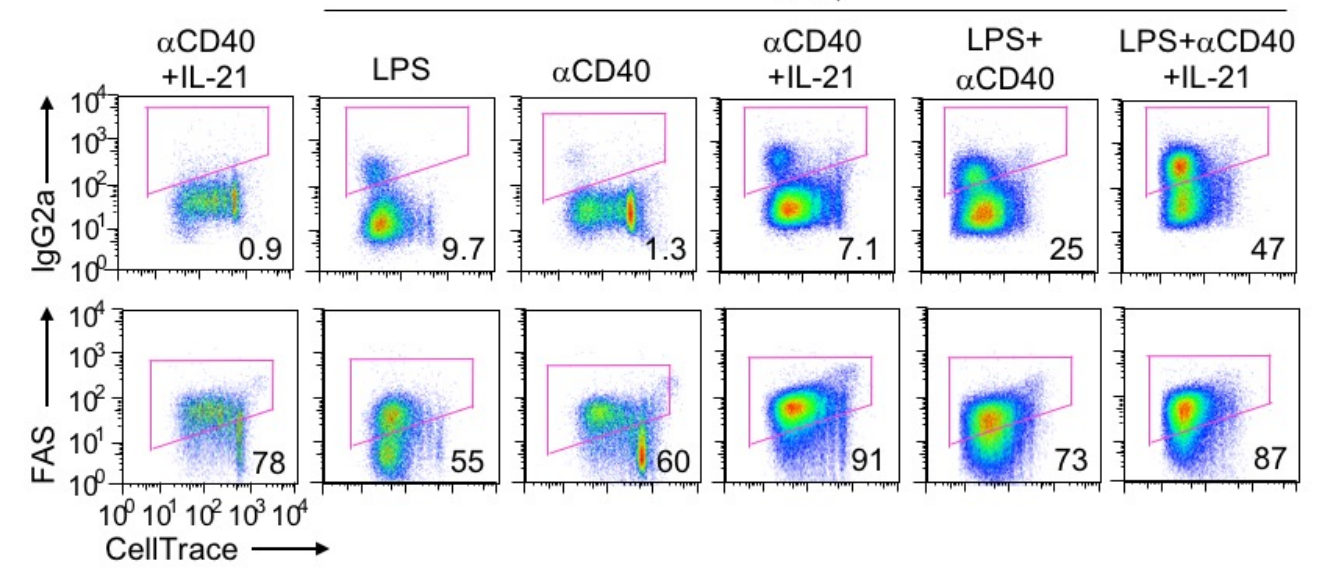

B

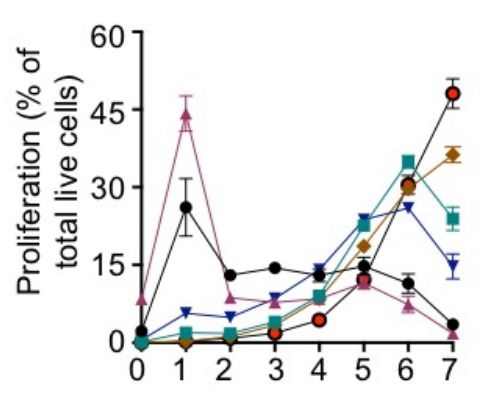

Cell division

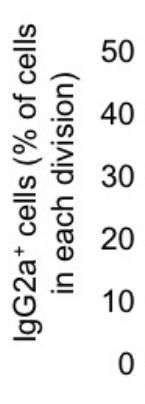$$
\text { (1) }
$$

- LPS $+\alpha C D 40+I L-21+I F N \gamma$

$\checkmark$ LPS $+\alpha \mathrm{CD} 40+\mathrm{IFN} \gamma$

$\leftarrow \alpha \mathrm{CD} 40+\mathrm{IL}-21+\mathrm{IFN} \gamma$

\section{fig. S25. IL-27-producing $B$ cells induce CSR}

proliferation/survival (B, left) and CSR to IgG2a (B, right) in B cells labeled with CellTrace $\mathrm{TM}^{\mathrm{TM}}$ and stimulated, as indicated, for $96 \mathrm{~h}$. Representative of two independent experiments. (C) Flow cytometry analysis of doublet formation by II27-GFP+ B cells (indicating IL-27+ B cells), as generated by stimulation with LPS plus CD154 and IL-21 for $24 \mathrm{~h}$, and their target B cells, i.e., B cells primed with LPS for $24 \mathrm{~h}$, after being mixed at different ratios for $1 \mathrm{~h}$. Representative of three independent experiments. (D) Flow cytometry analysis of CSR to IgG2a in IL-27-producing (CD45.1 $1^{+}$B cells, as generated in vitro by stimulation with LPS plus CD154 for $24 \mathrm{~h}$, and (CD45.2+) B cells, as primed by LPS for $24 \mathrm{~h}$, after being mixed and cultured for additional $72 \mathrm{~h}$. Representative of three independent experiments.

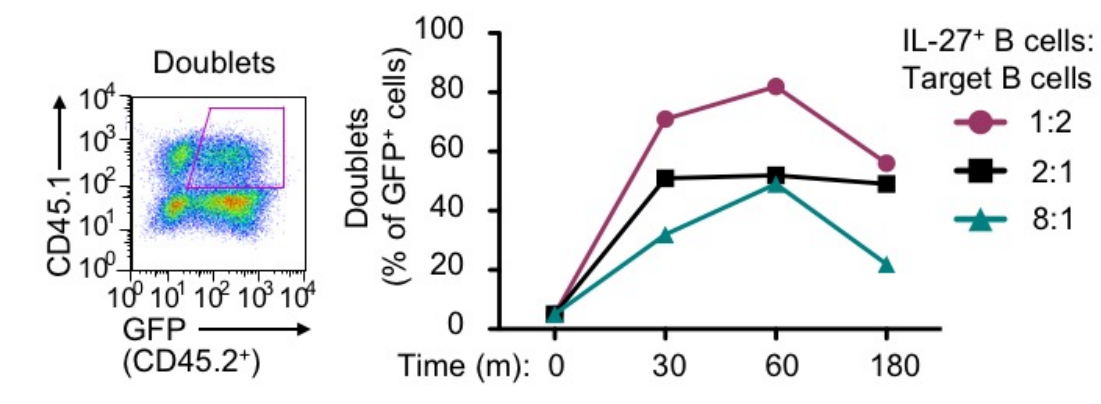

D
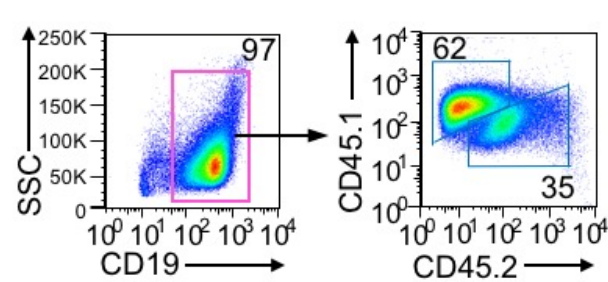

IL-27+ B cells:

CD 45. +'; $^{+}$

+LPS+CD154

(0-24 h)

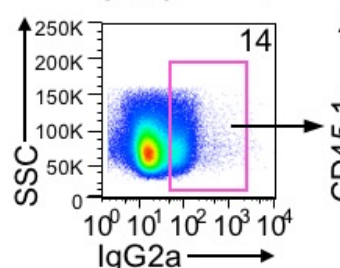

CD45.2

+LPS

Mixing: at $24 \mathrm{~h}$; Analysis: at $96 \mathrm{~h}$ 

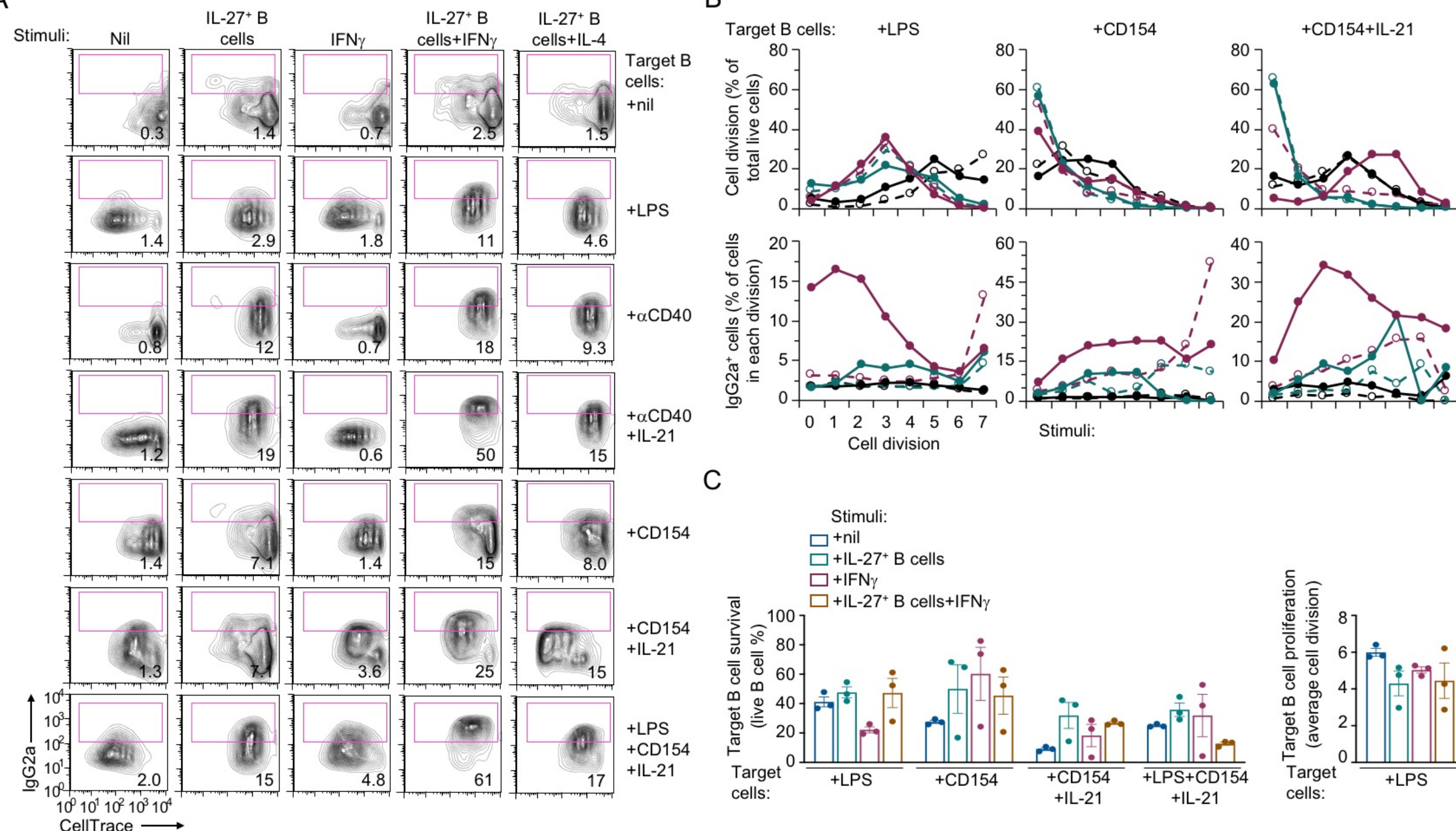

+LPS+CD154+IL-21
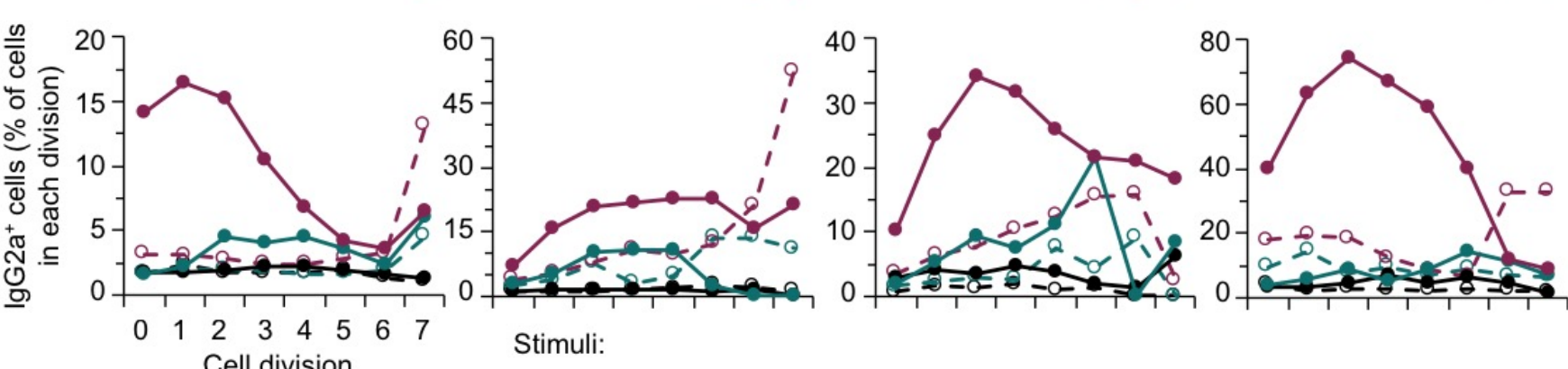

C

$$
\text { Cell division }
$$
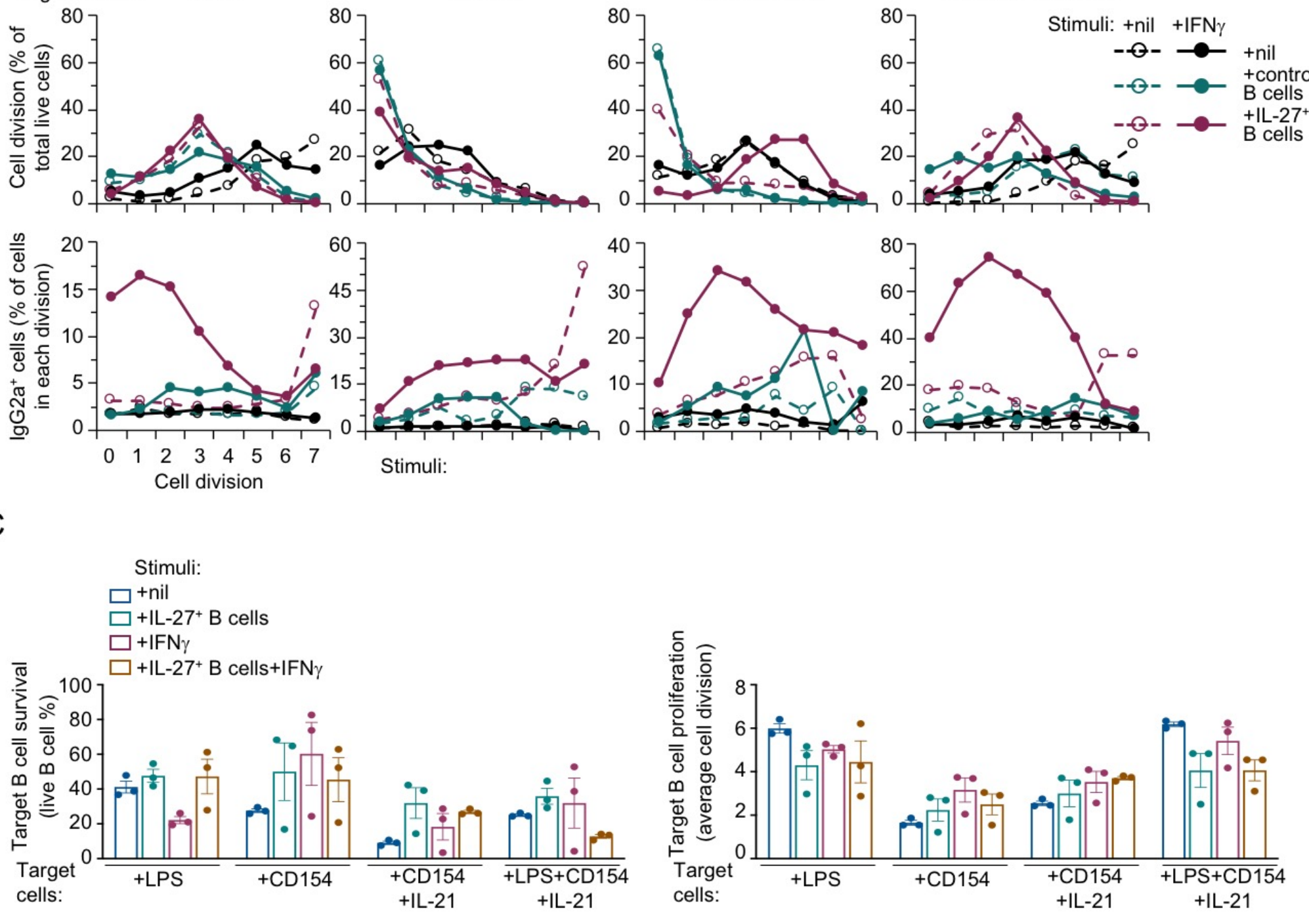

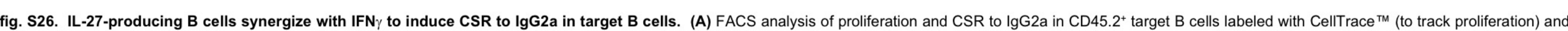

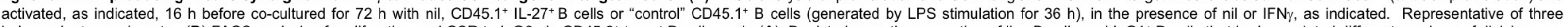

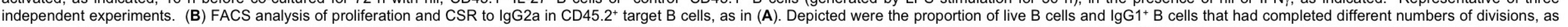

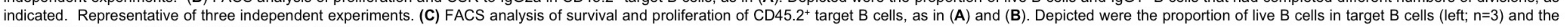
average numbers of divisions of target $B$ cells (right; $n=3$ ). 